\title{
Unmet need for family planning in developing countries and implications for population policy [Arabic]
}

John B. Casterline

Population Council

Steven W. Sinding

Follow this and additional works at: https://knowledgecommons.popcouncil.org/departments_sbsr-pgy

Part of the Demography, Population, and Ecology Commons, Family, Life Course, and Society Commons, and the International Public Health Commons How does access to this work benefit you? Let us know!

\section{Recommended Citation}

Casterline, John B. and Steven W. Sinding. 2000. "Unmet need for family planning in developing countries and implications for population policy," Policy Research Division Working Paper no. 135 [Arabic]. New York: Population Council. https://doi.org/10.1111/j.1728-4457.2000.00691.x 
الاحتياجات غير الملباة إلى تنظيم الأسرة في البلدان النامية ودلالاتها بالنسبة للسياسات السكانية

إعداد

جون ب. كاسترلاين(John B, Casterline) ستيفن و. سيندنج(Steven W. Sinding)

2000 رقم 135 


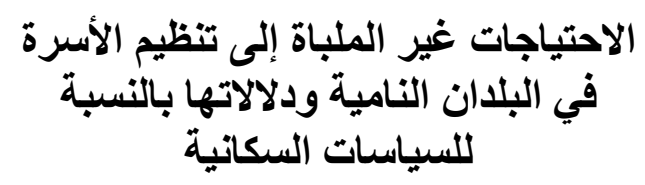

إعداد

جون ب. كاسترلاين (John B. Casterline)

ستيفن و. سيندنج(Steven W. Sinding)

جون ب. كاستر لاين (John B. Casterline) شريك أول في مجلس السكان، قسم بحوث السياسات،

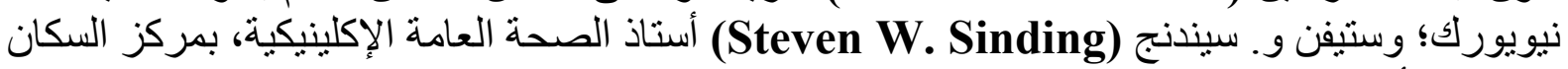

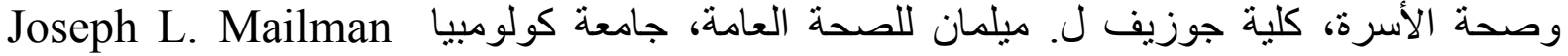
School of Public Health, Columbia University

أجريت هذه الدراسة بدعم من المنح المقدمة إلى قسم البحوث حول السياسات من الجهات التالية: مؤسسة

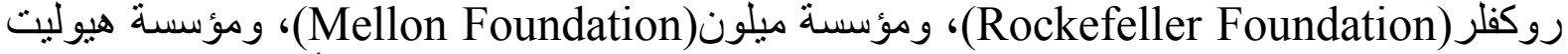
(Hewlett Foundation)

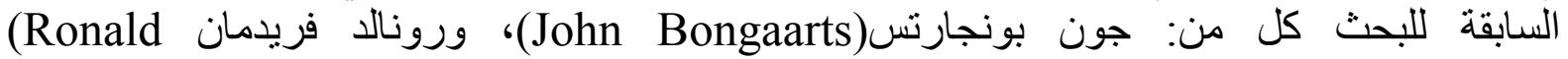
Freedman) وجيمس ماكارثي (James McCarthy)، ونثارلز وستوف(J) (Charles Westoff). 
تعد الاحتياجات غير الملباة إلى تنظيم الأسرة مفهوما محوريا في الخطاب السكاني الدولي على مدى فألى

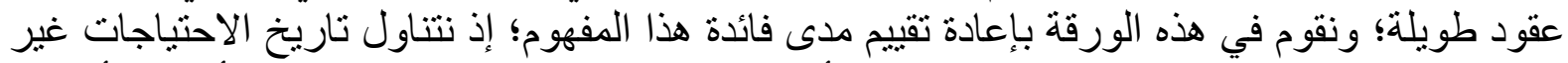

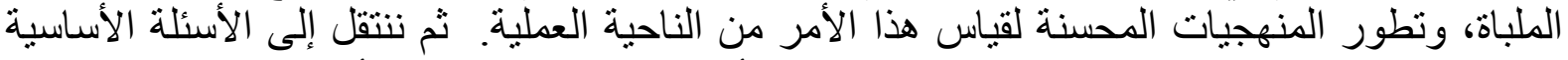

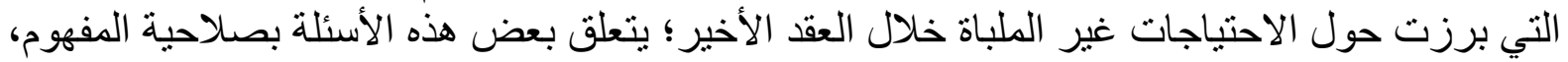

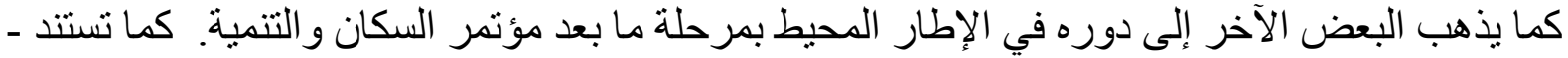

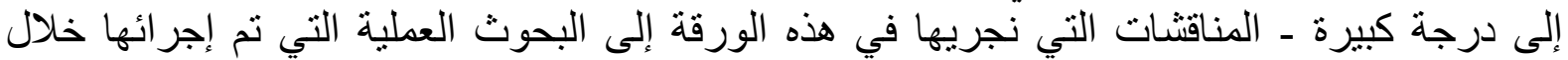

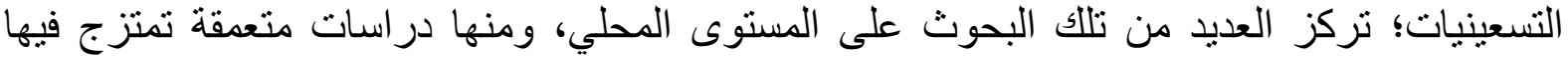

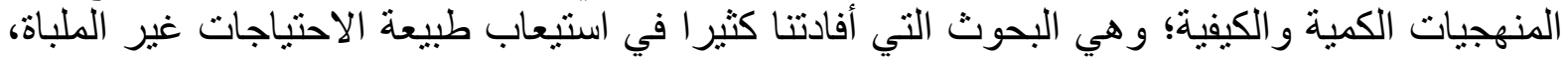

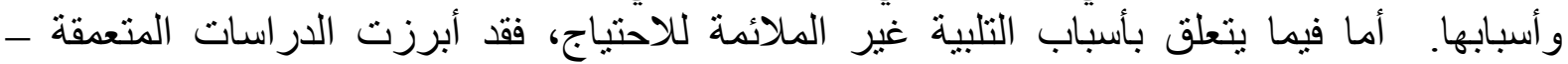

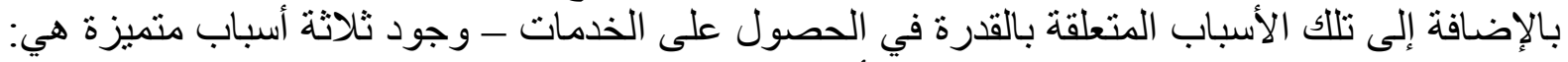

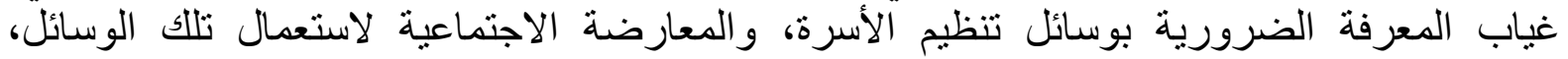

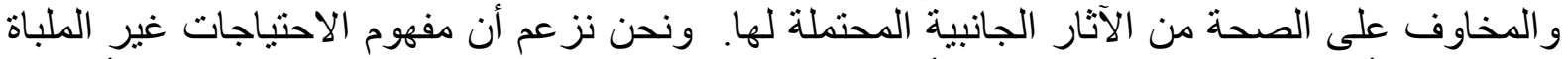

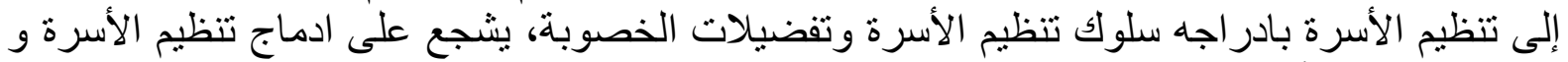

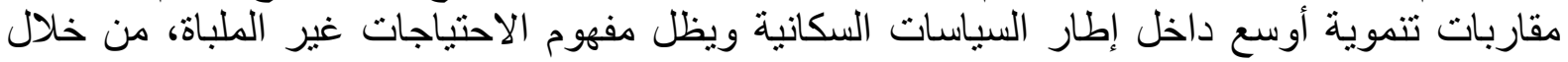

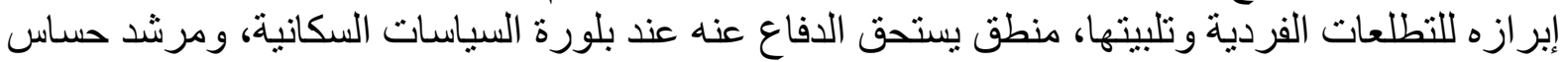
في تصميم بر امج تتظيم الأسرة.

لا يمكن إعادة نشر المادة المتضمنة في هذه الورقة دون الحصول على تصريح مسبق من المؤلفين. 


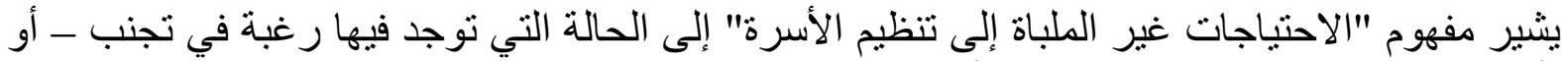

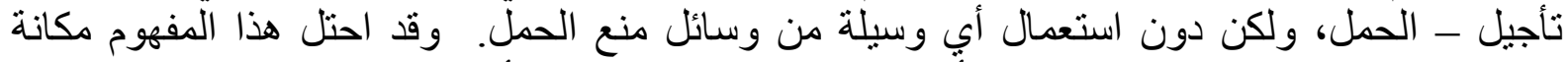

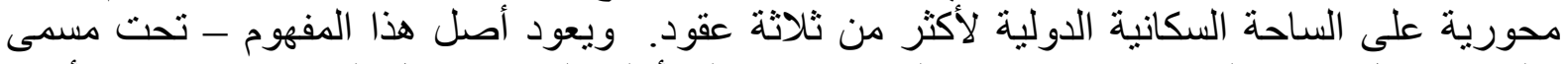

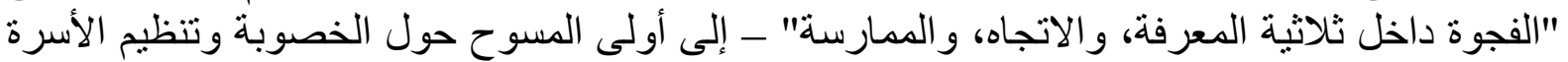

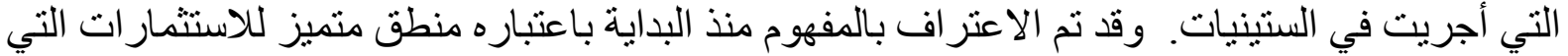

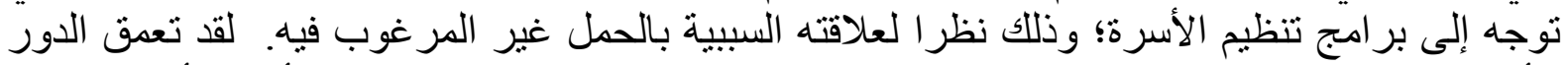

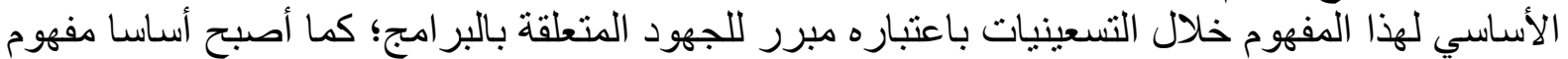

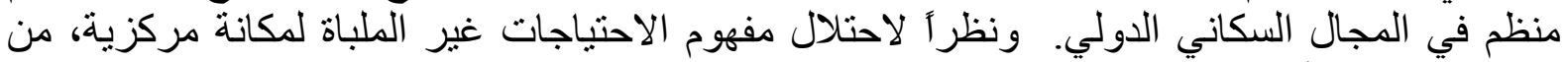

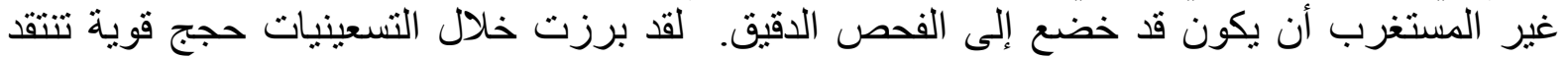

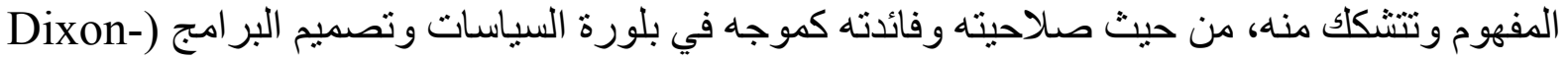
Mueller and Germain 1992; Pritchett 1994: Jain 1999 عدد مهم من البحوث الجديدة المبنية على الملاحظة والاختبار حول الاحتياجات غير الملباة إلى تنظيم الإنيا

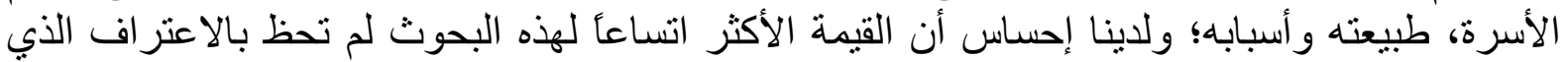

يتمثل هدفنا هنا في إعادة النظر في فائدة مفهوم الاحتياجات غير الملباة إلى تتظيم الأسرة، باعتباره مفهوم

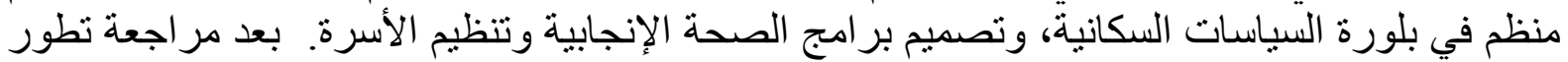

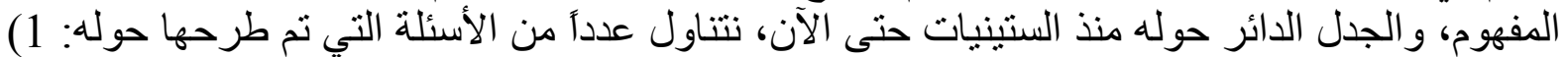

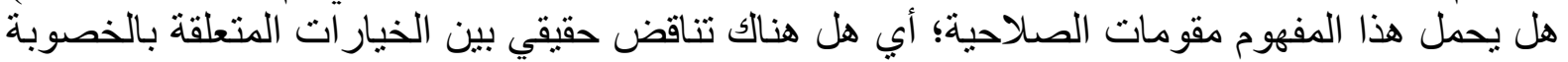

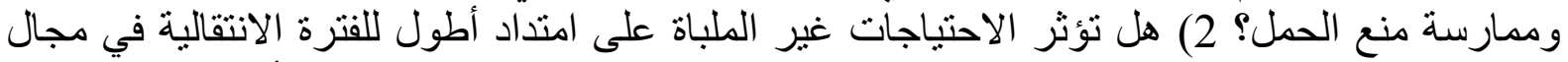

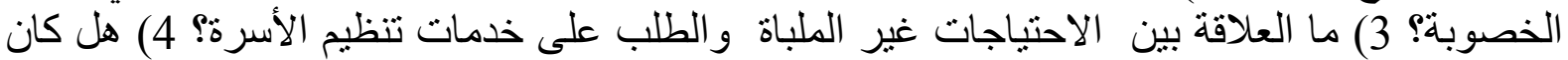

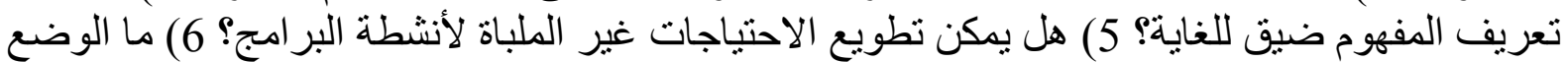

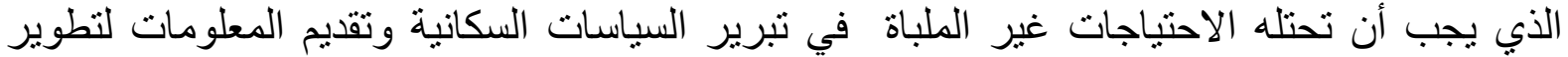

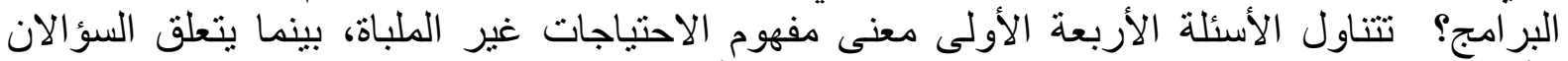

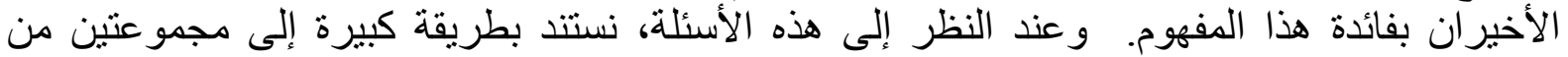

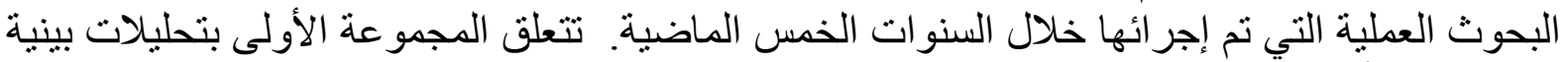

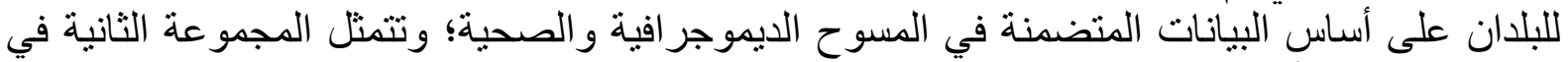

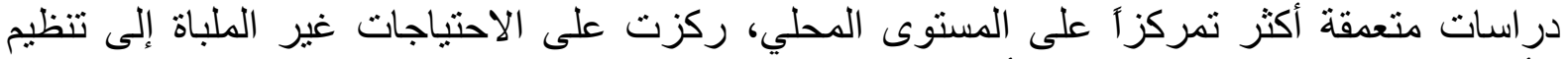

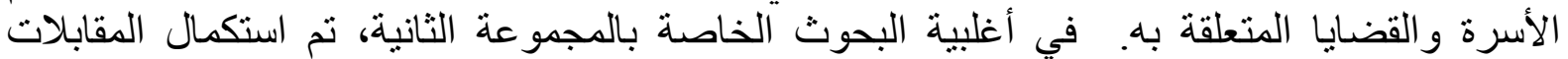

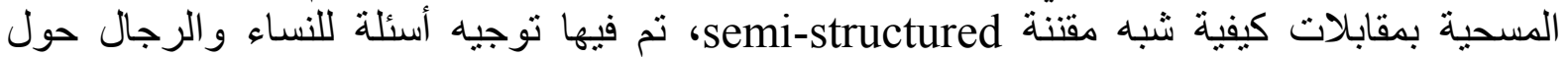

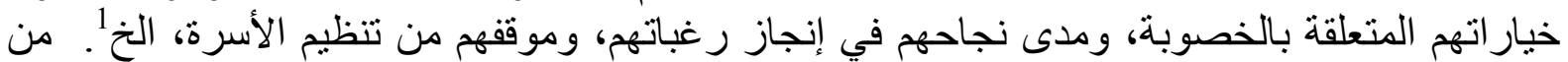

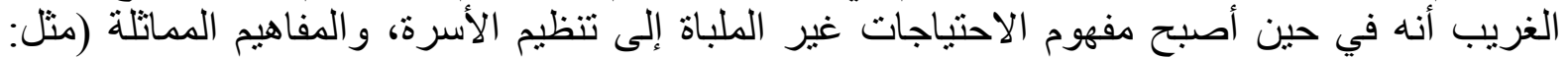

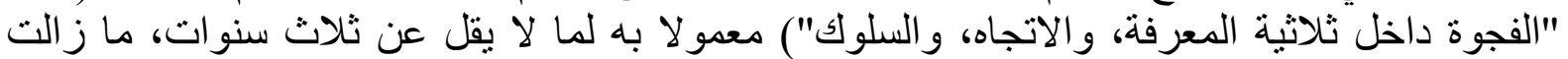

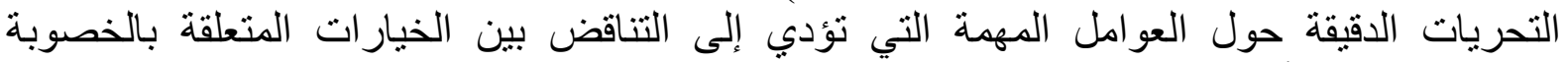
وممارسة تنظيم الأسرة، من المجالات البحثية الحديثة نسبيا.

\section{خلقية عامة}

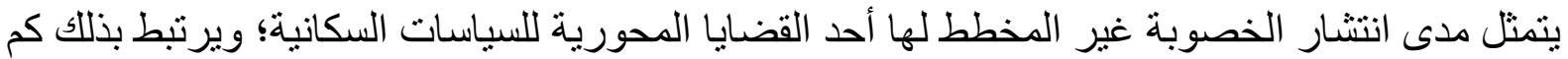

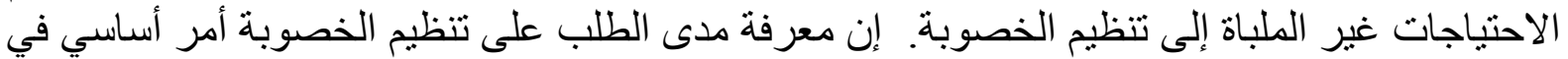

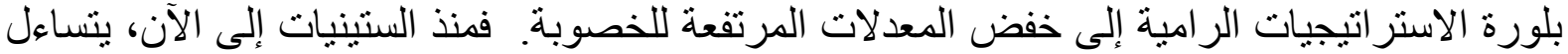

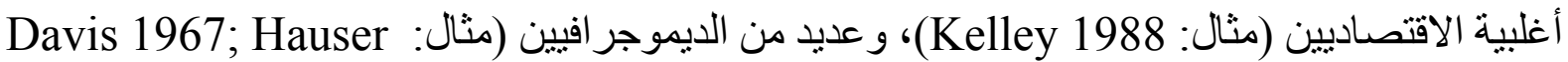
1969) إذا كان هنالك كم كبير من الطلب على تنظيم الخصوبة لم يتم تلبيته بطريقة ملائمة في البلدان ذات 
المعدلات المرتفعة من الخصوبة، مما يجيز نوفير خدمات تنظيم الأسرة، أو تبني مقاربة لتوفير أثكال

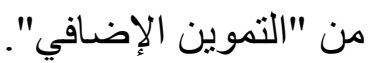

من أجل تناول هذه الثكوك، وتحديد مدى الطلب على تتظيم الخصوبة، تم إجراء المسوح حول المعرفة، التهاء

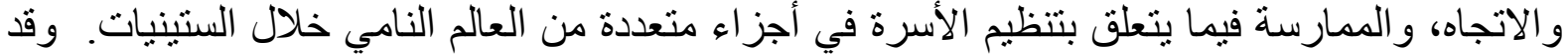

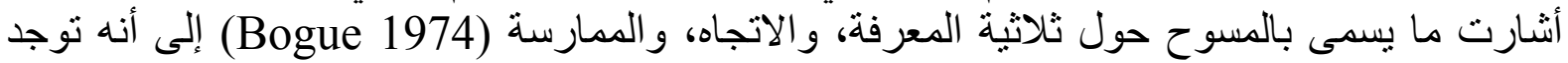

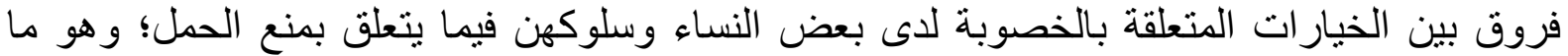

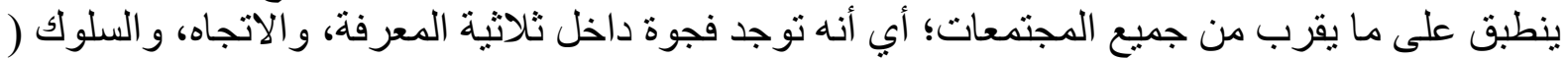
1969

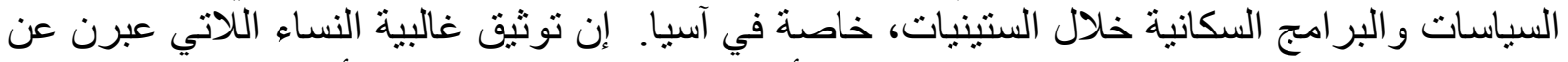

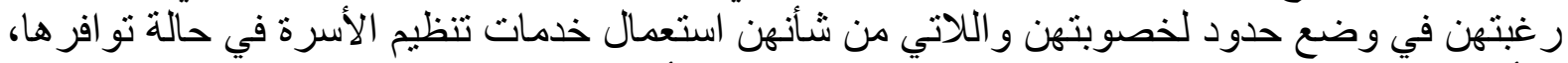
قد ألهم عديد من الحكومات لإطلاق بر امج طموحة لتنظيم الأسرة.

و على الرغم من ذللك، ما زال هنالك تشكك حول الطلب الفعلي على خدمات تنظيم الأسرة. قام تام رونالد

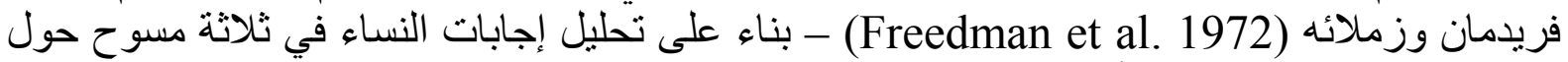

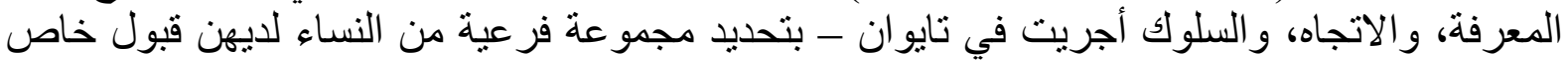

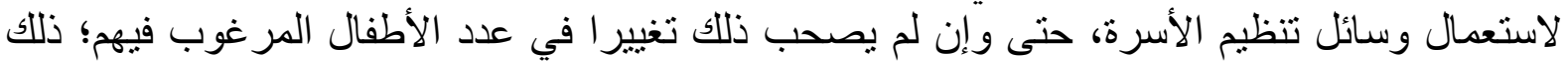

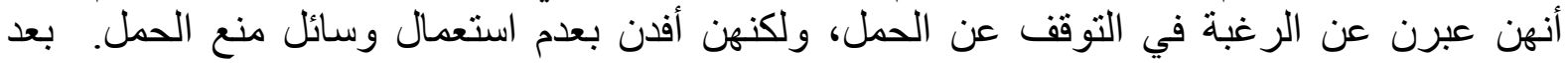

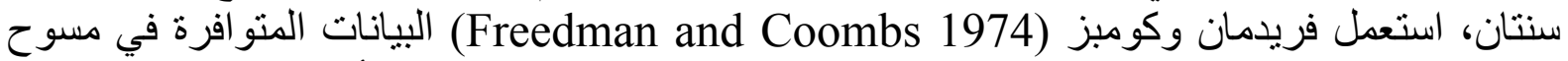

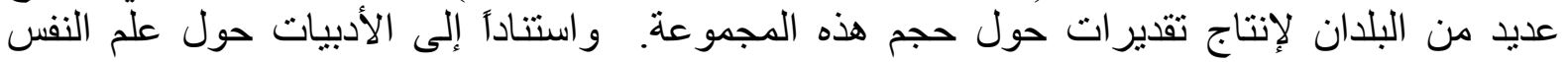

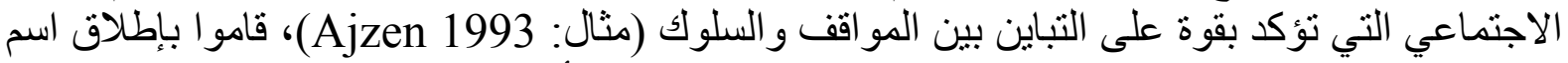
"السلوك المتتاقضي" على الفجوة الموجودة بين الحاجة إلى تنظيم الأسرة، و الممارسة الفعلية.

بعد المسوح حول ثلاثية المعرفة، و الاتجاه، و الممارسة التي أجريت في الستينيات، جاء برنامج المستح

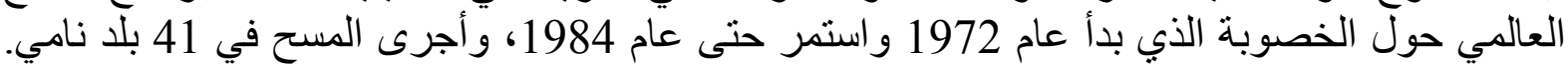

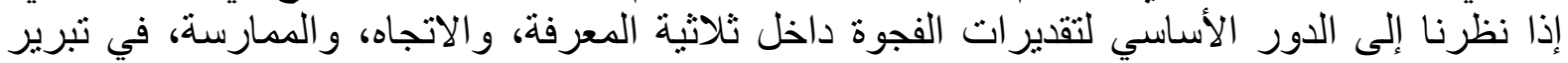

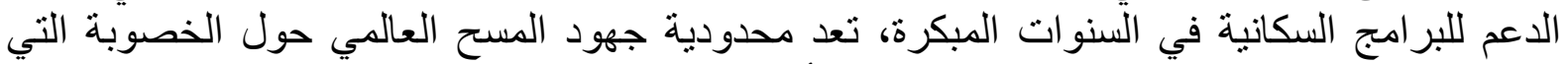

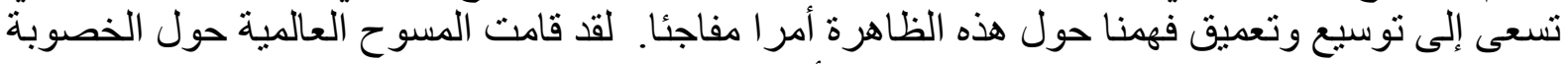

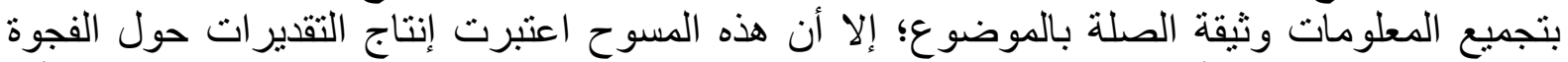

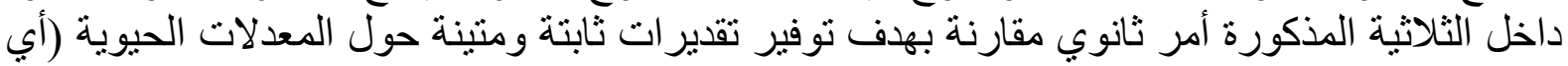

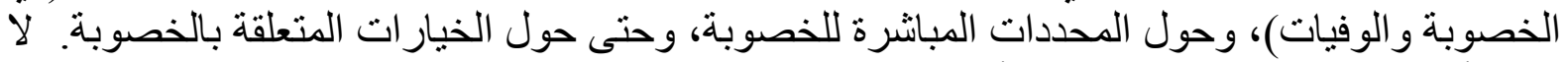

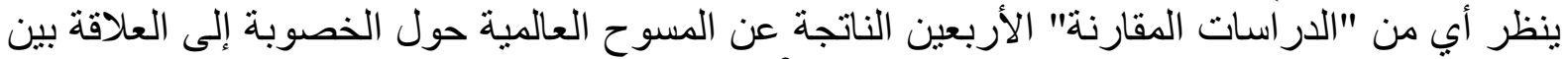

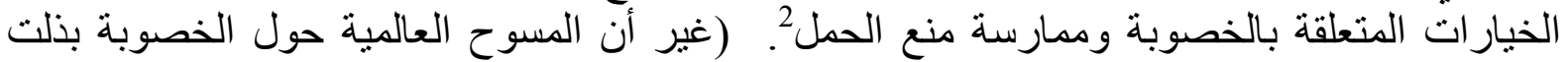

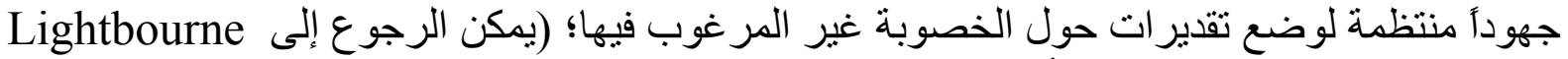

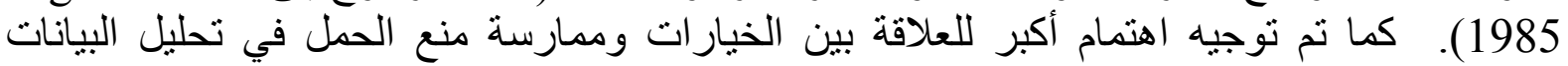

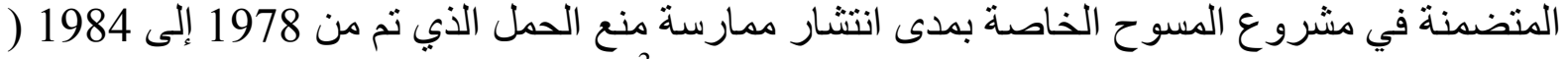
.3 (Anderson and Morris 1981; Morris et al. 1981

حينما أمكن الإطلاع على المجموعة الأولى من المسوح العالمية حول الخصوبة في آسيا، قام وستوف (لإنى

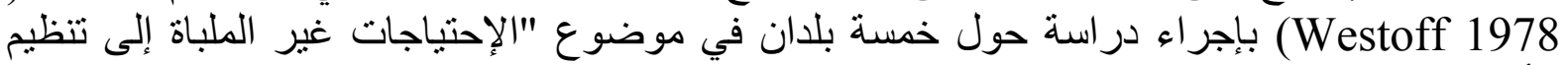

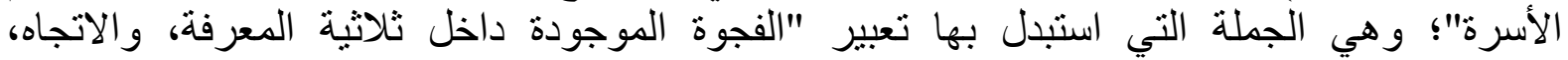

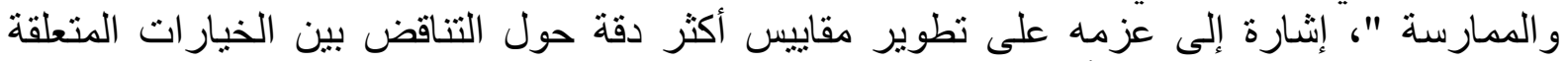

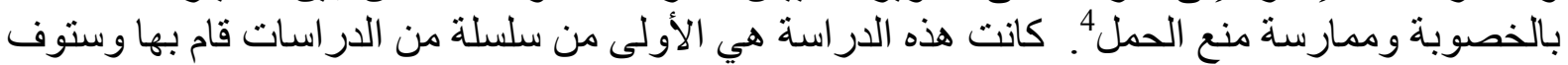




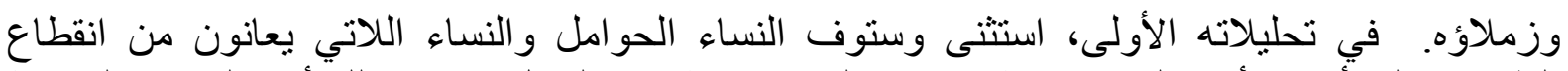

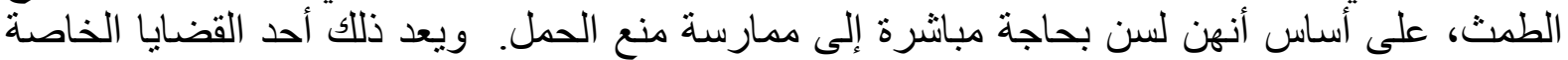

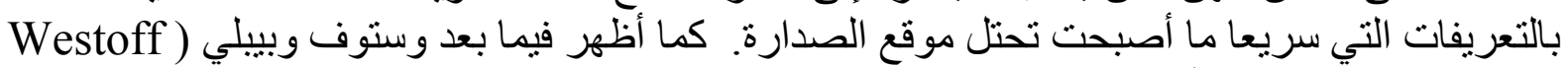

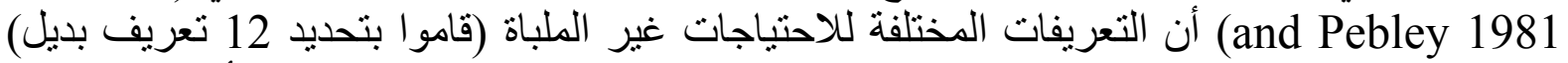

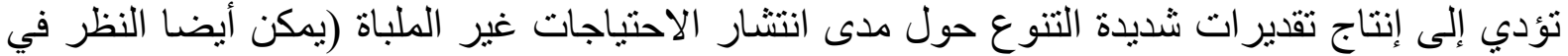

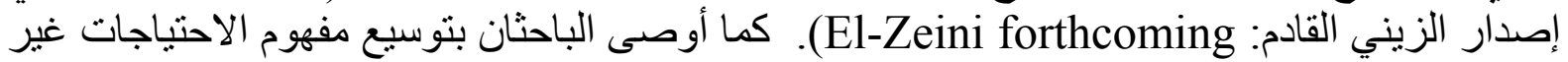

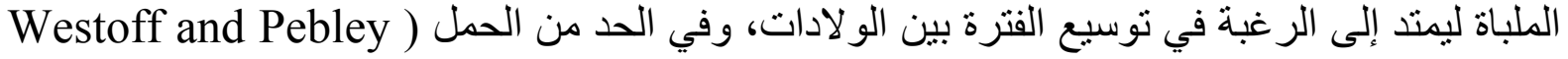

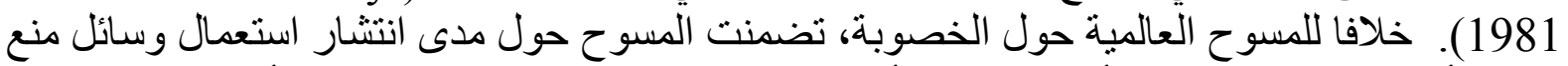

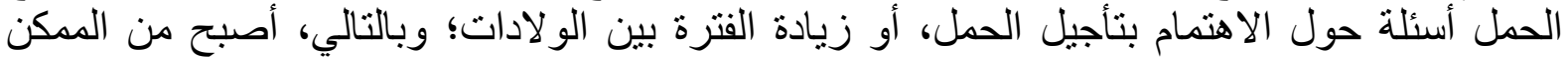

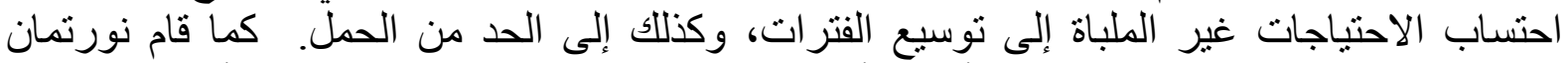

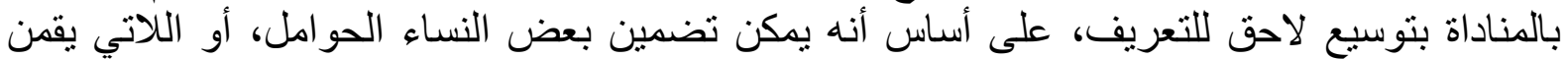

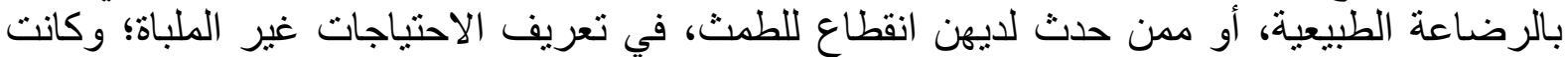

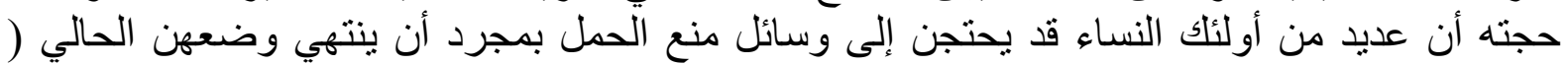

لقد أصبحت المسوح الديموجر افية والصحية وسائط لنرسيخ تلك التعريفات المنطورة حول قياس

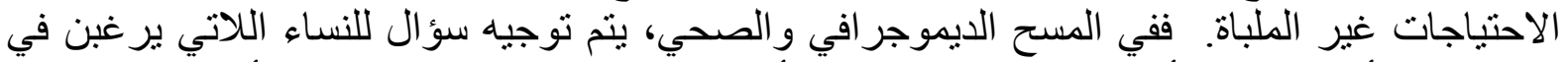

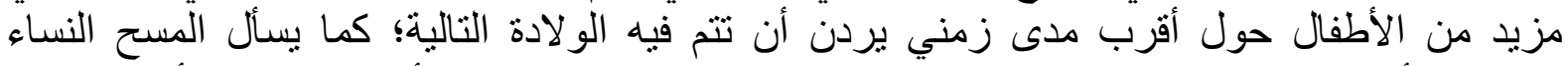

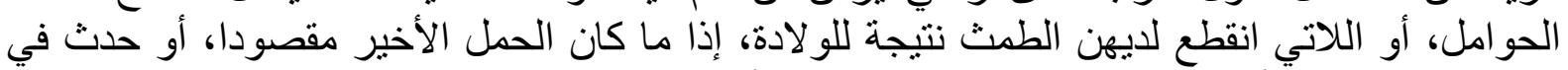

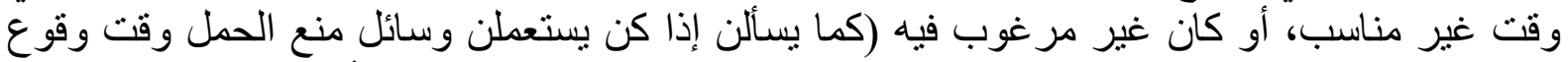

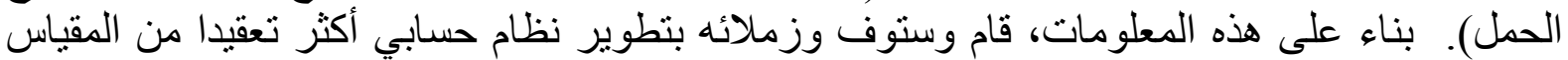

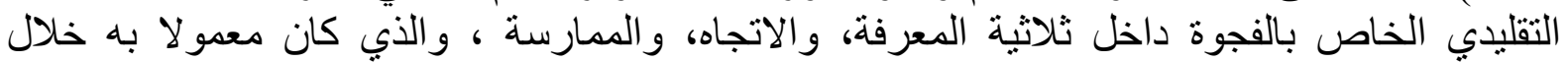

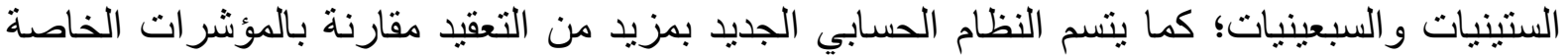

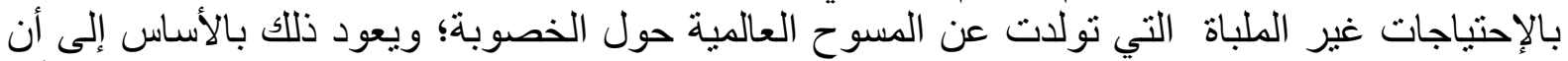

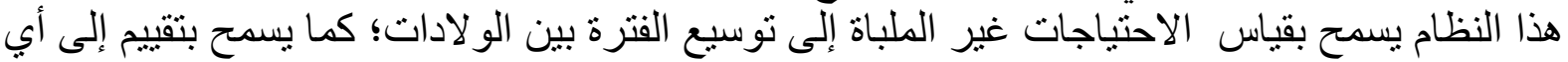

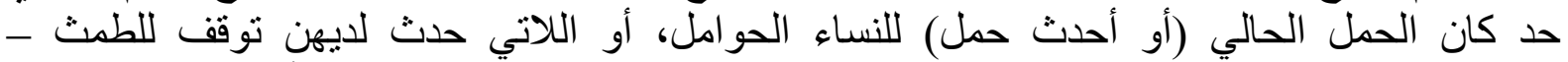

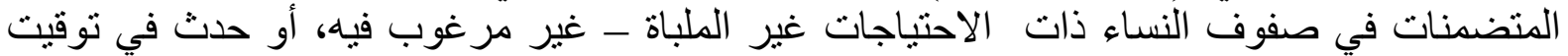

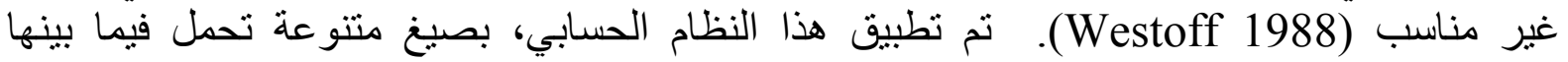

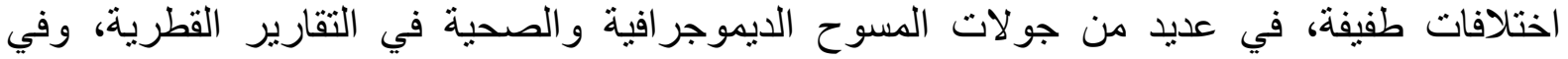

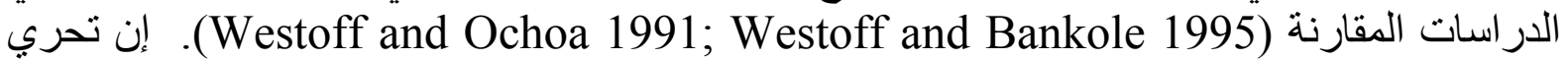

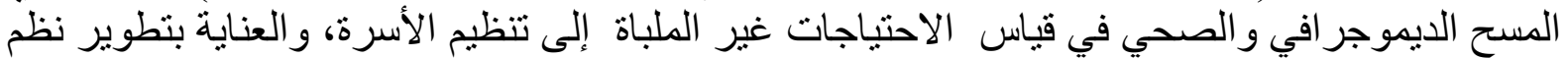

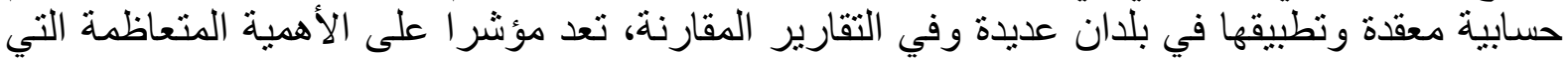

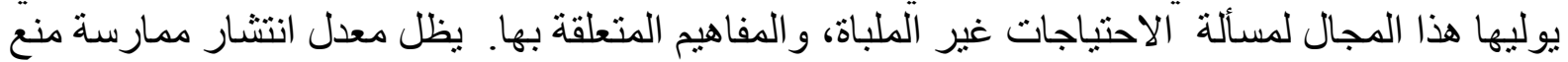

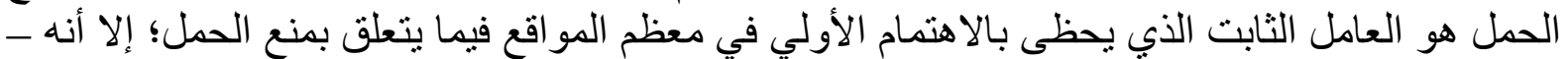

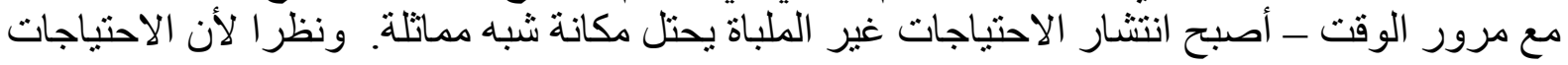

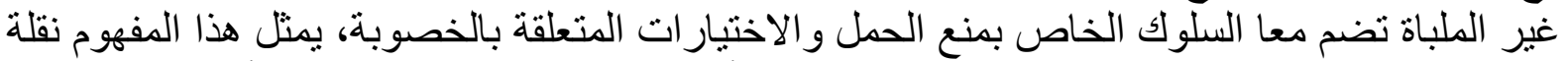

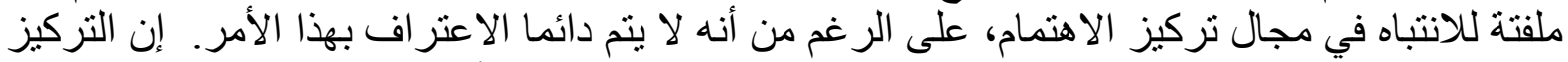

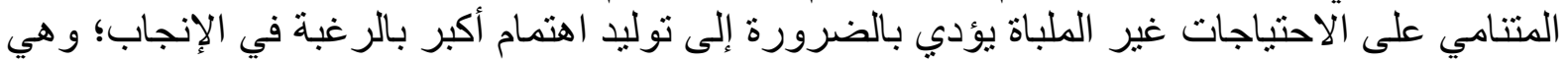
نقطة سوف نعود إليها فيما بعد.

في بداية التسعينيات، أصبح مفهوم التلبية الاحتياجات غير الطلباة مفهوما أساسيا في الأدبيات الخاصة الإنة

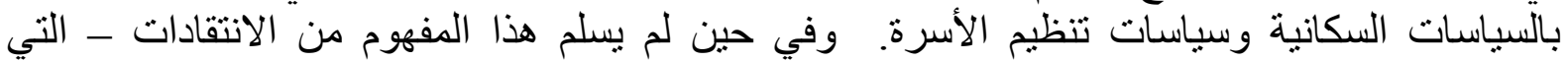

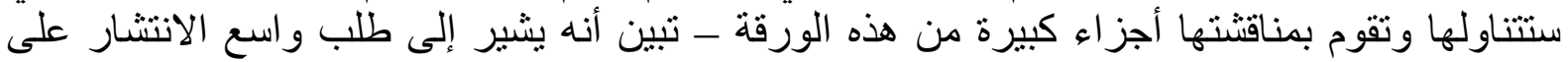




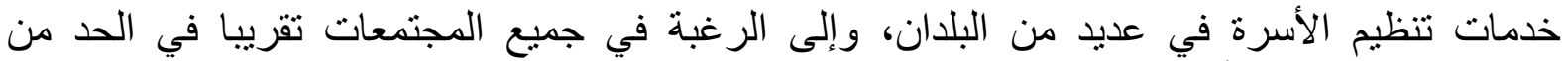

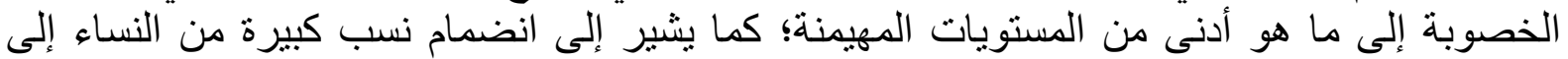
صفوف من أنجبن حديثأ نتيجة لحمل غير مرغوب فيه (Lightbourne 1985; Adetunji 1998).

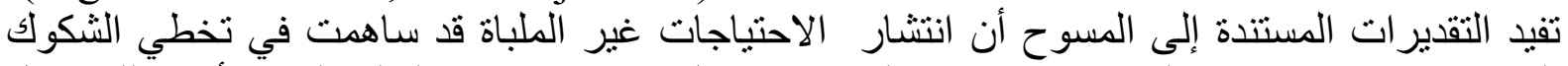

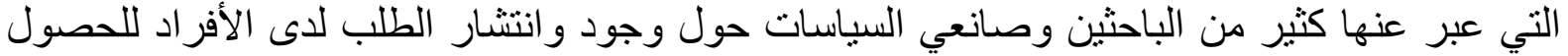

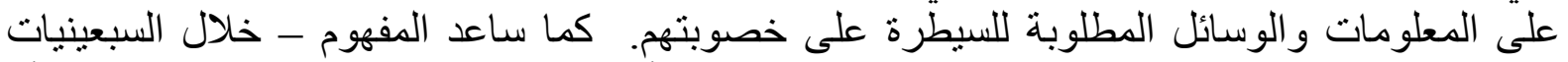

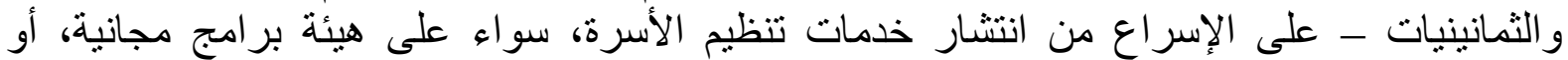

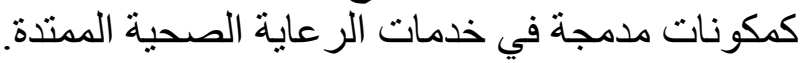

ومع التحضير للمؤتمر الدولي للسكان والتنمية (1994)، بدأت مجموعات دفاعية advocacy معنية

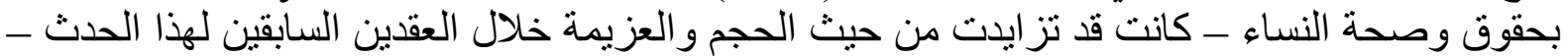

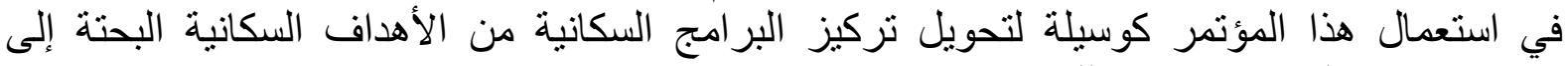
Sen et al. 1994; McIntosh and Finkle ) الأهتمام بحياة النساء، بما في ذلك صحتهن الإنجابية

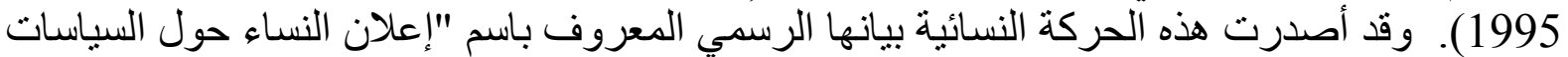

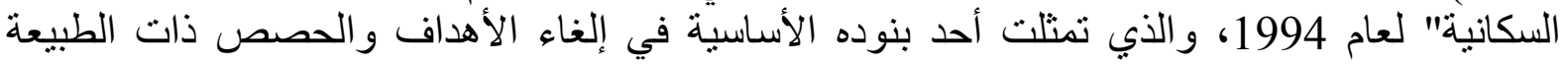

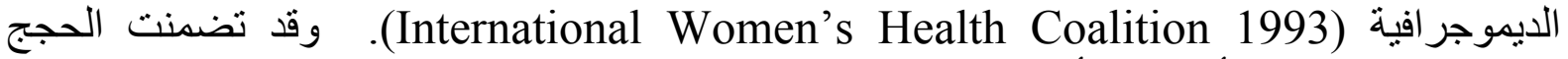

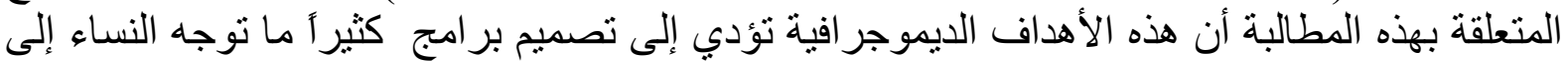

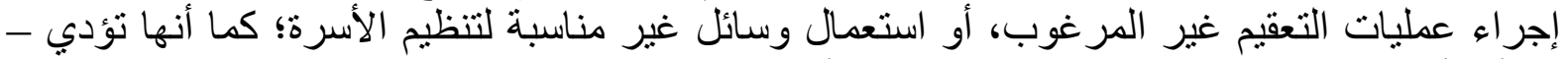
في أسوأ تجلياتها - إلى إكر اه النساء على التعقيم أو الإجهاض (1994 Garcia-Moreno and Claro

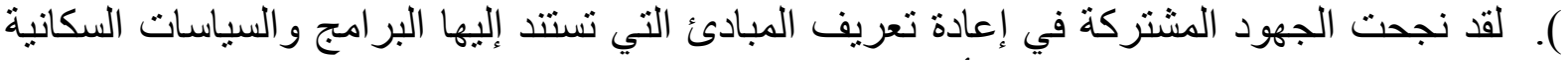
الدولية خلال المؤتمر المذكور؛ وهو الأمر الذي انعكس بطريقة واضحة في في خطة فئة عمل المؤتمر الدولي

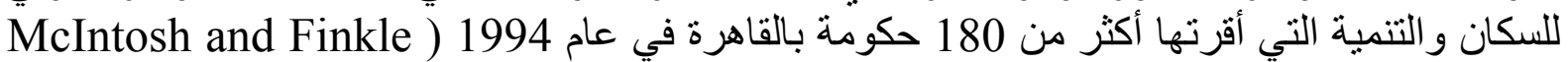

وبالرجوع إلى الماضي، نجد أن الأعمال التحضيرية للمؤتمر الدولي للسكان والتنمية، وفعاليات المؤتمر

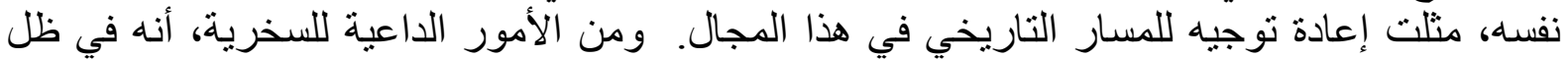

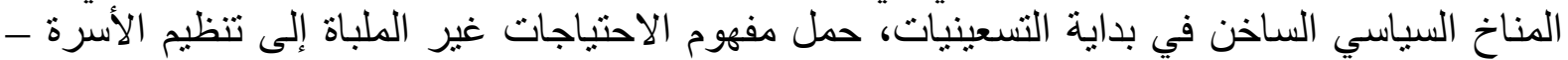

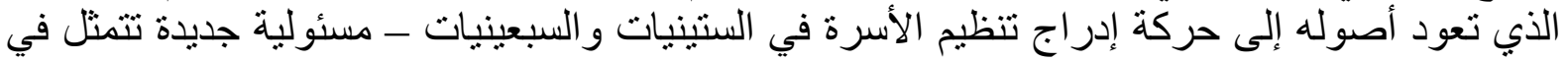

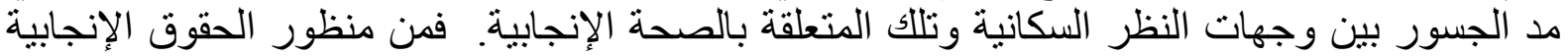
للنساء، تم اعتبار الاحتياجات غير الملباة كمؤشر لانتهاك هذه الحقوق، وأحد المبادئ الأساسية لتمكين

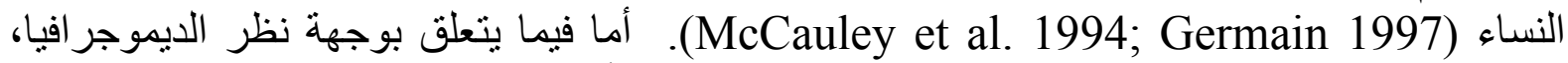
فيشير تحليل منشور عام 1994 (Sinding et al. 1994) إلى أن التلبية الكاملة للاحتياج إلى منع الحمل فئل

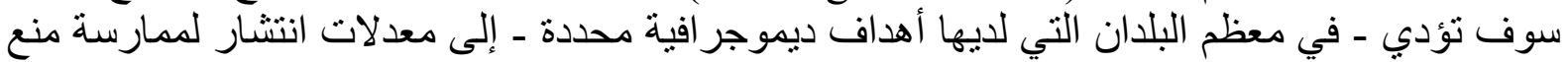
الحمل تفوق الأهداف التي وضنعتها لنفسها. وقد تمثل التفسير المستند إلى هذا الاستنتاج في أن السياسات

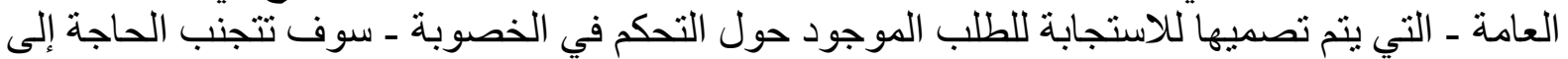

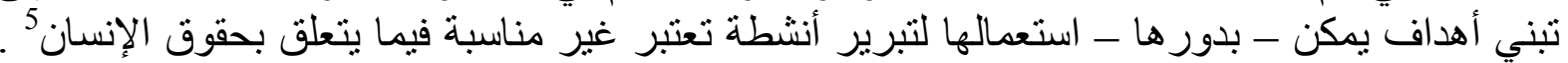

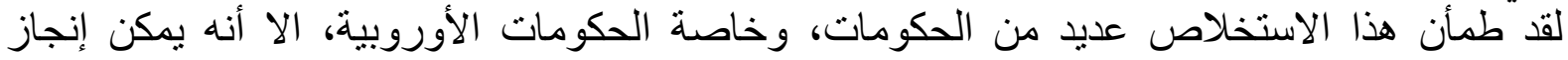

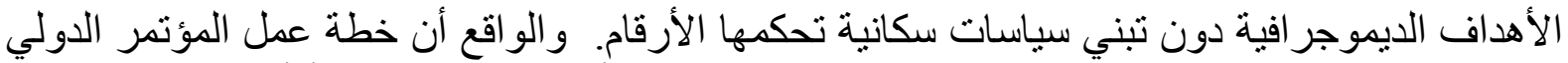

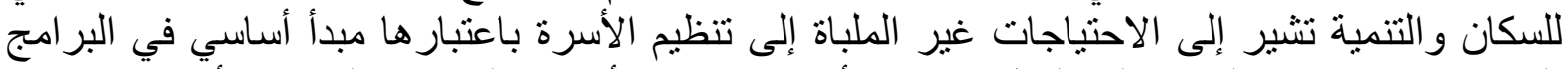

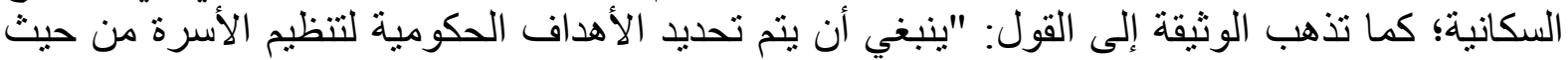
الاحتياجات غير الملباة إلى الحصول على المعلومات والخدمات..كما يجب أن أن تقوم جميع البلدان خلال

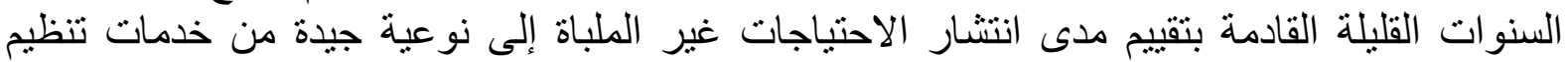
الأسرة على المستوى الوطني.." (United Nations 1994: paragraphs 7.12 and 7.16). لقد مدات 
أصبح التقليل من انتشار الاحتياجات غير الملباة هدفا في حد ذاته، بدلا من أن يظل وسيلة لتحقيق الأهداف الديموجر افية (Sai 1997).

وفي إدراك متأخر عن الحدث، يبدو أن الفترة المحيطة بمؤتمر القاهرة مثلت علامة فارقة بالنسبة

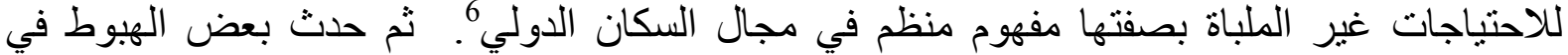

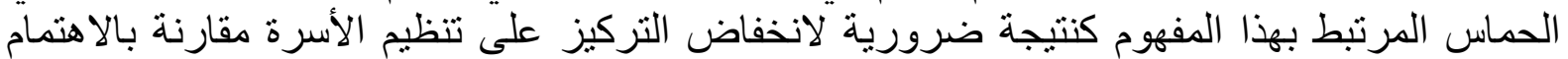

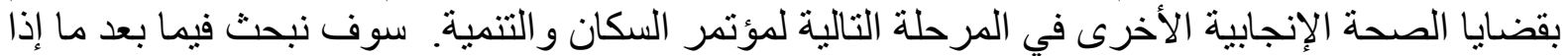

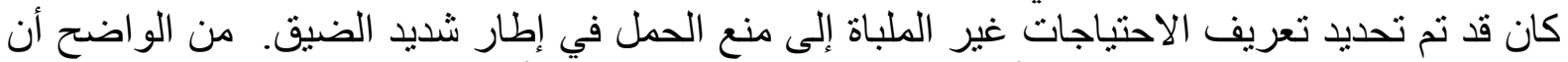

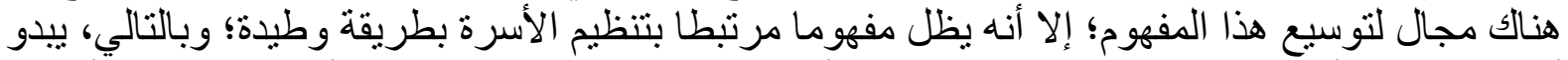

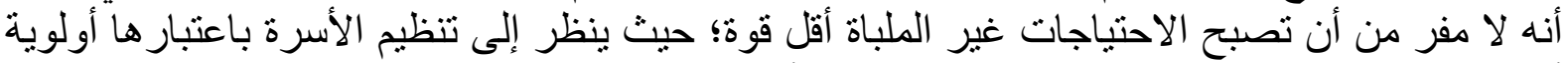

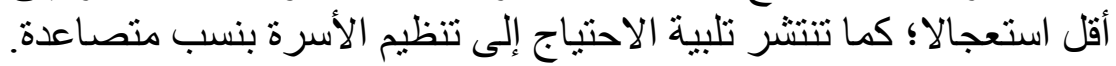

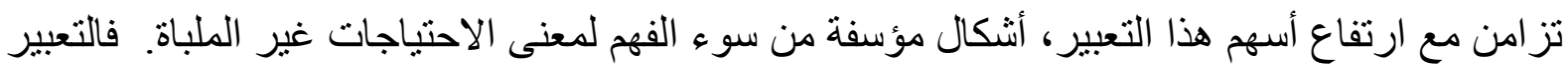

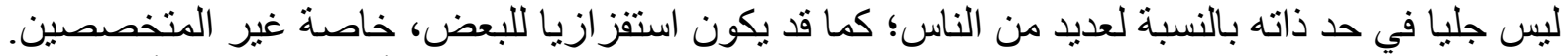

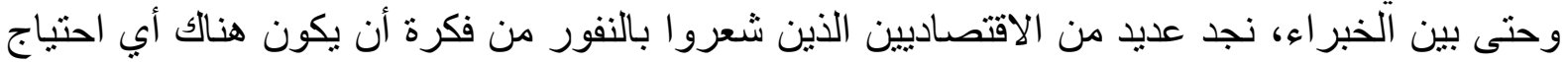

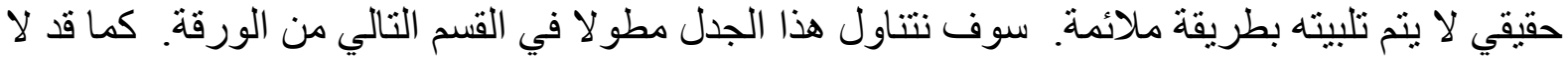

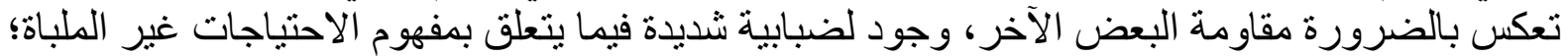

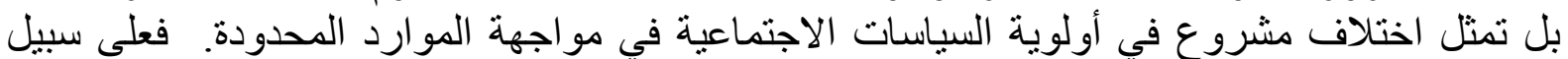

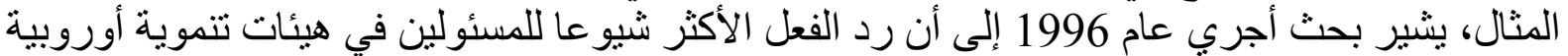

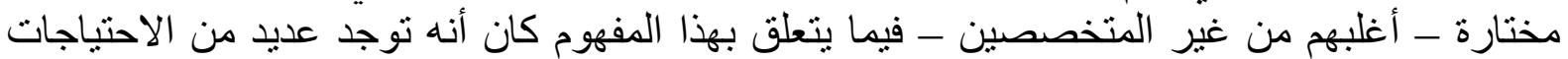

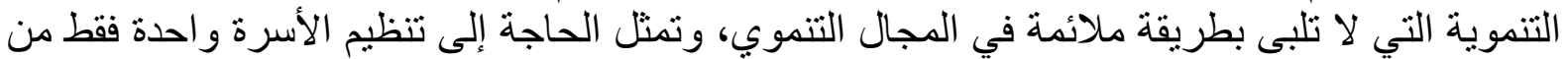
بين هذه الاحتياجات (Market and Opinion Research International 1997).

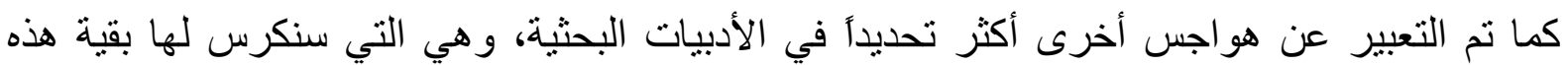

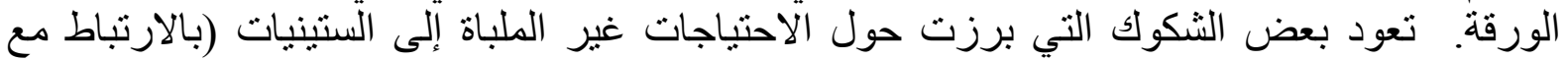

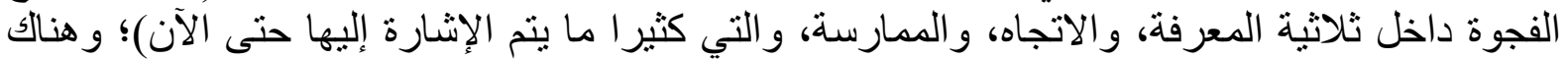

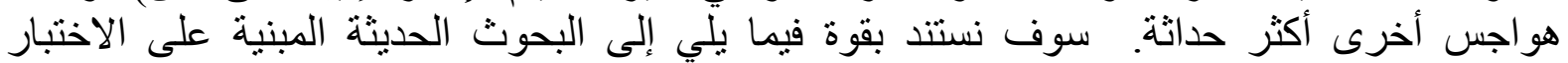

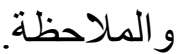

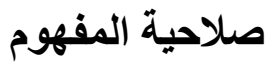

قد يكون أكبر تحدي لمفهوم الاحتياجات غير الملباة هو الزعم بأنه لا يرتبط بظاهرة سلوكية صالحة؛ إلئه

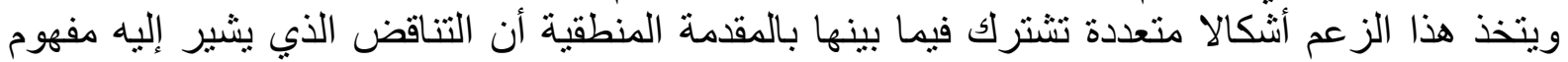

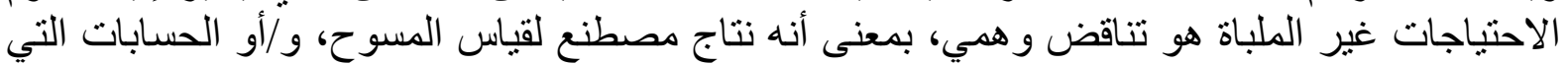

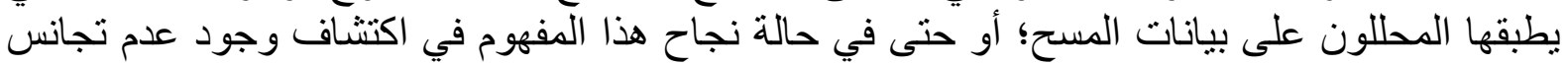

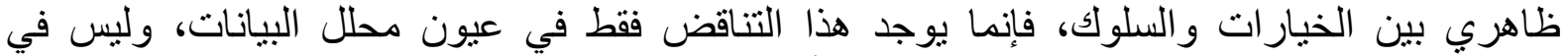

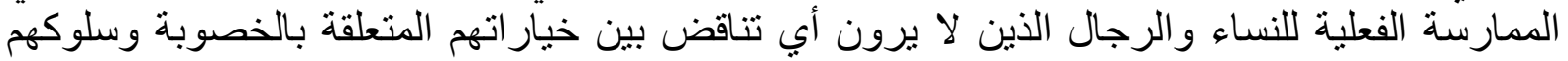

الإنجابي.

تذهب نسخة أولية لهذه الحجة إلى رفض قياس الاختيار ات المتعلقة بالخصوبة على أساس المسوح؛ وهي

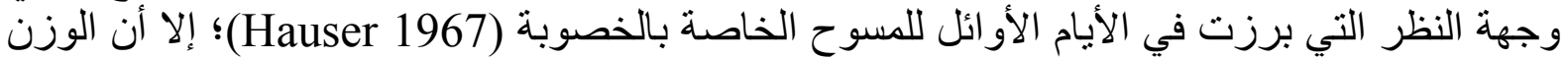

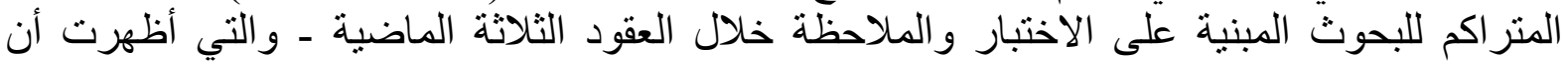

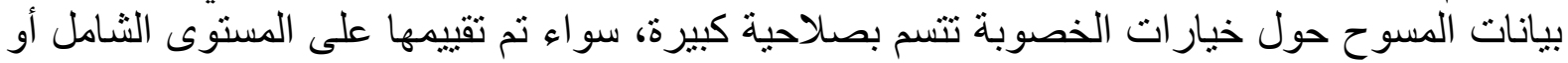


Westoff and Ryder 1977; Hermalin et al. 1979; Westoff) الفردي - قد دحض هذه الحجة في 1998 يقم حتى الآن أهم المشتبكين في الجدل الدائر حول الاحتياجات غير الملباة بمساءلة الصلاحية الإجمالية

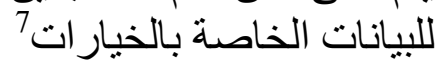

وبدلا من ذلك، انتقلت المناقثة إلى التساؤل حول وجود علاقة بين الاحتياجات غير الملباة وخبرات

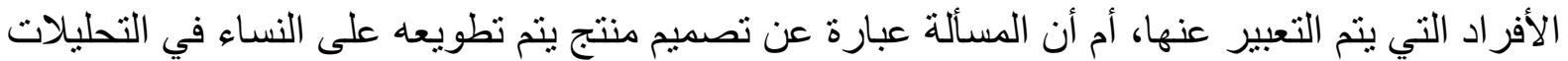

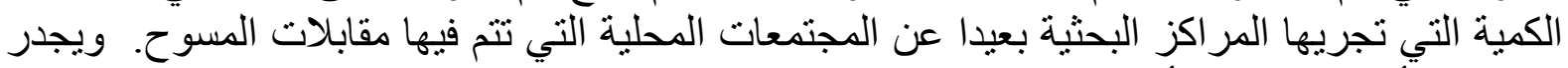

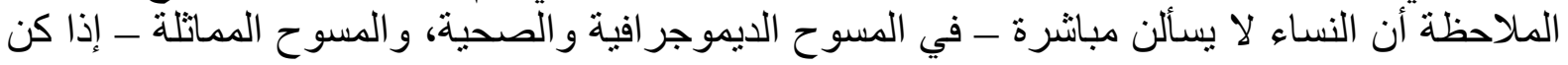

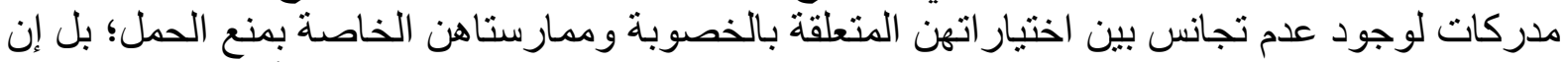

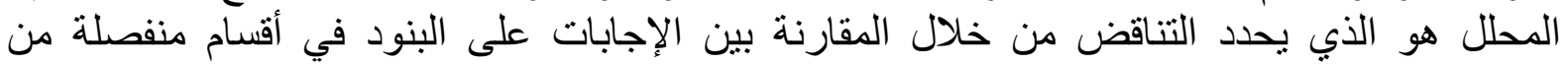

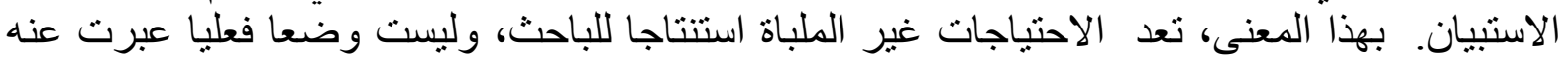

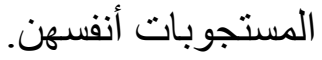

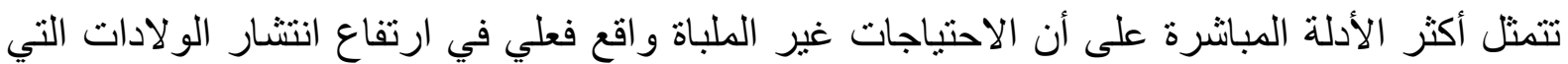

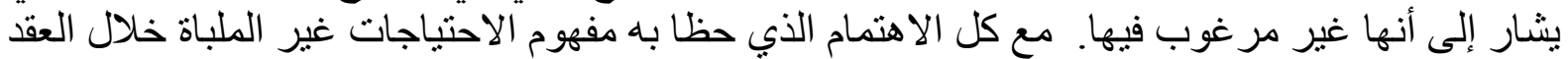

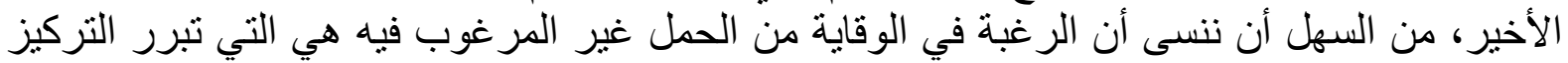

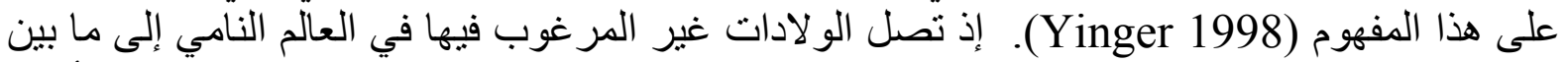

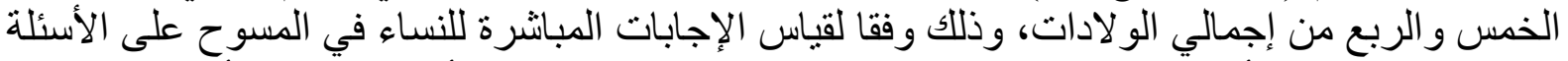

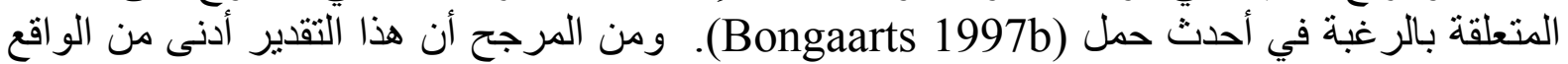

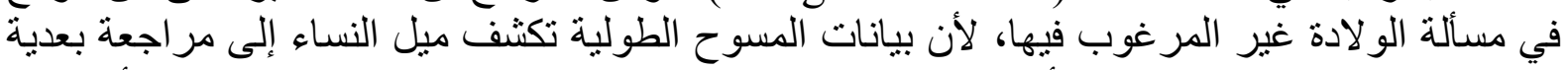

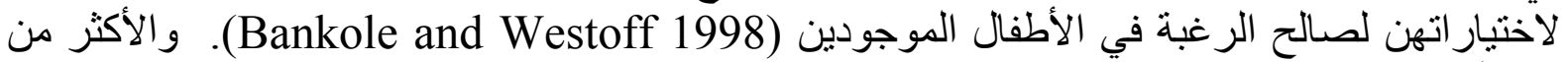

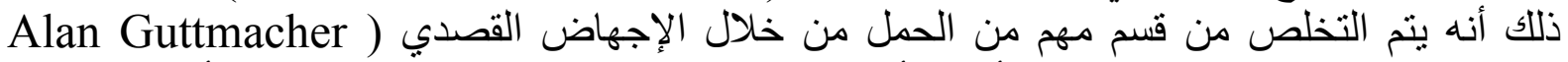
1999

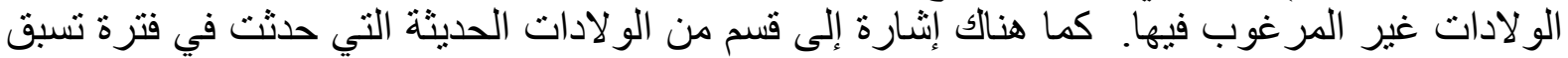

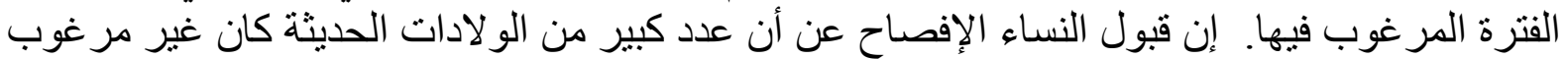

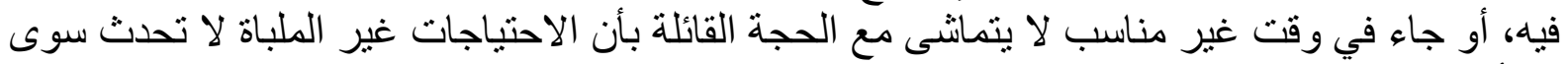
في أذهان الباحثين وصانعي السياسات.

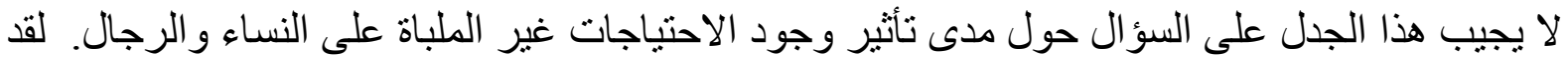

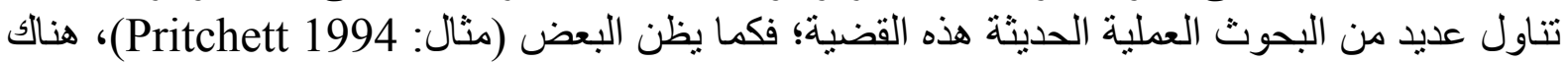

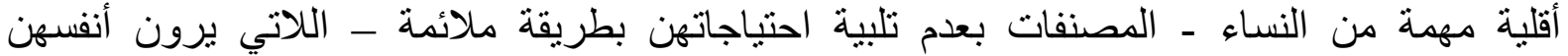

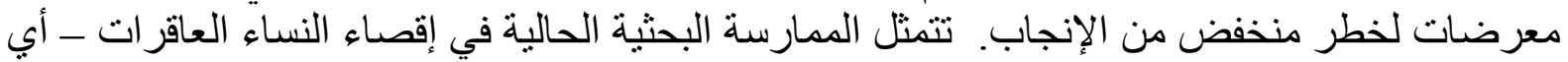

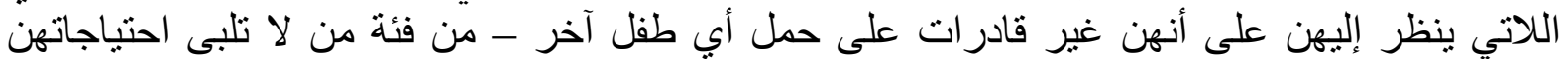

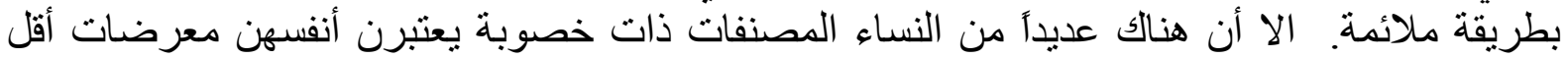

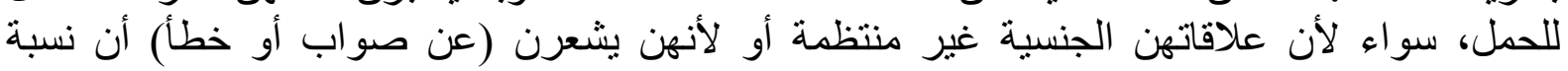

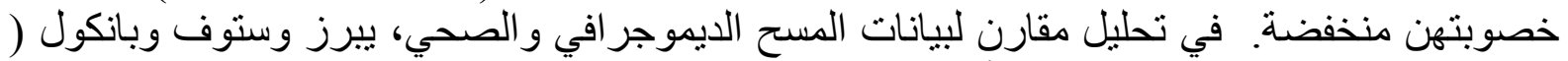

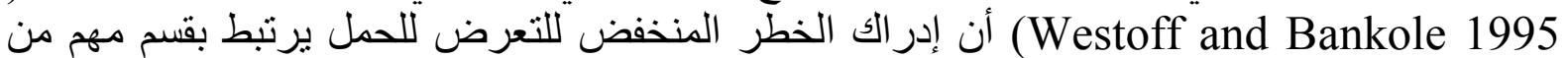

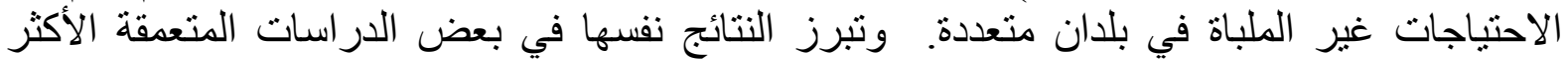

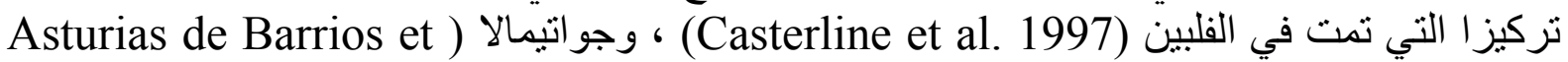
al. 1998 (al-Z ومصر (El-Zanaty et al. 1999)، ونيبال (Stash 1999)؛ إلا أن هذه النتائج تبدو أقل

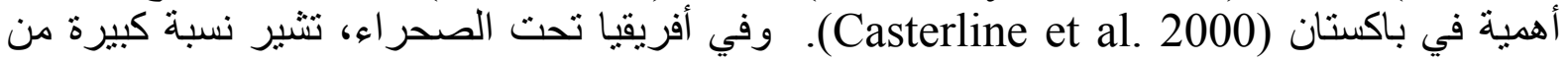
النساء المصنفات بين من لا تلبى احتياجاتهن بطريقة ملائمة فيما يتعلق بتوسيع الفترة بين الو لادات، إلى كن 
أنهن في حالة من الامتناع الجنسي في مرحلة ما بعد الولادة (Westoff and Bankole 1995).

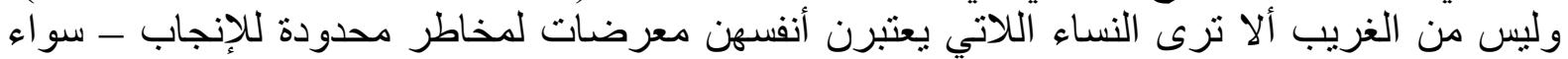

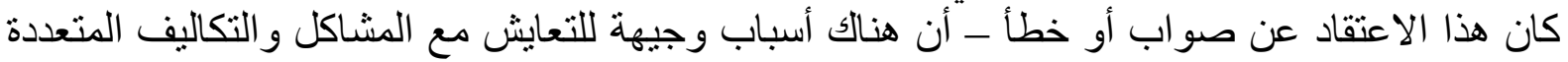

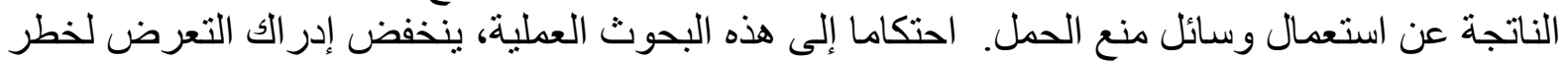

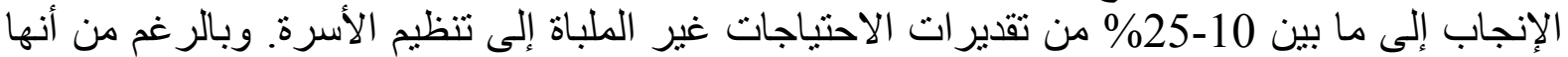
نسبة غير بسيطة، إلا أنها غير كافية لاحض المفهوم.

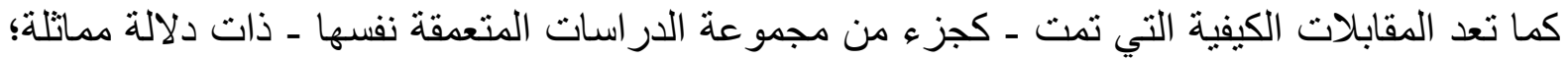

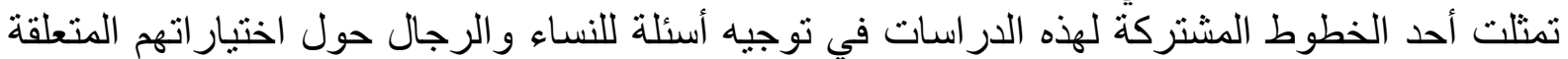

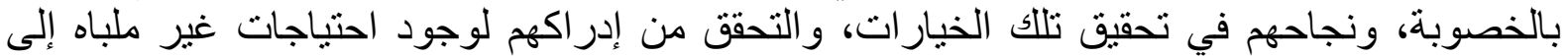

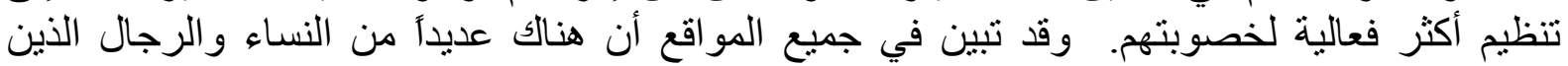

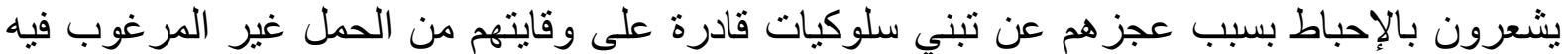

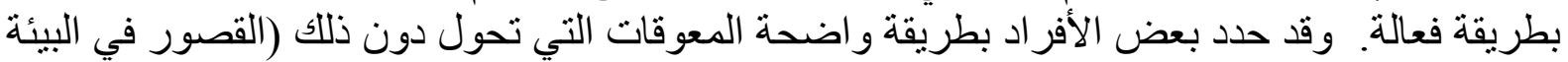

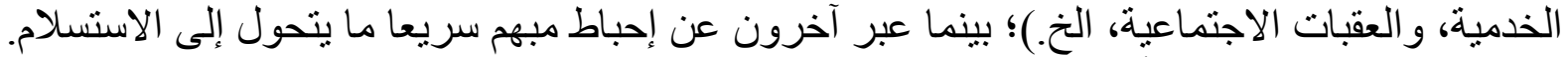

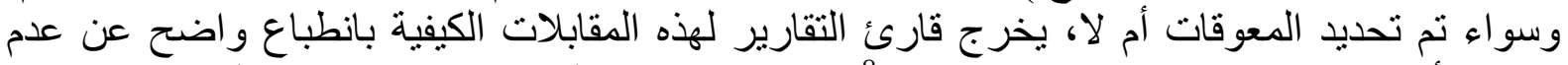

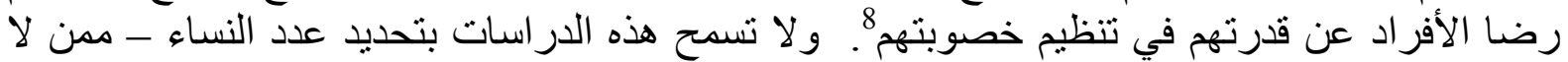

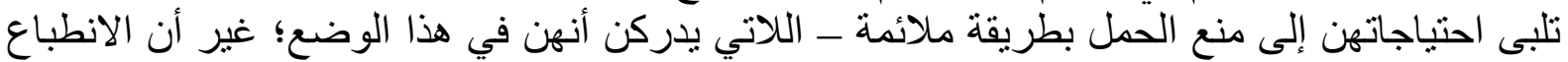

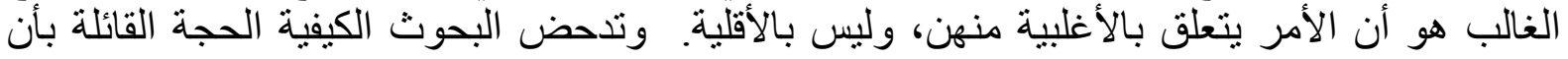
الاحتياجات غير الملباة عبارة عن مجرد خيال قام بتصميمه محلل المسح.

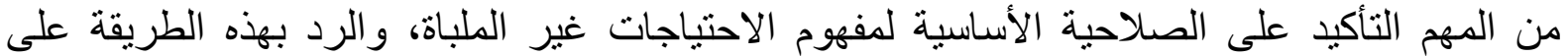

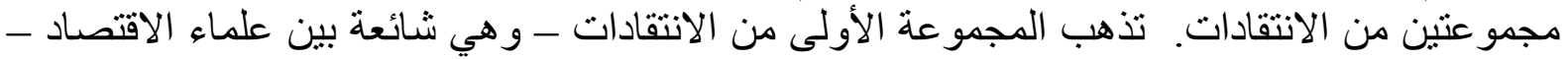

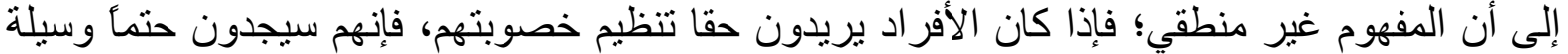

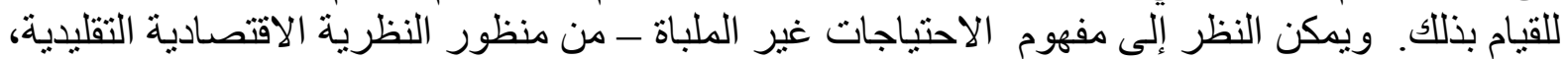

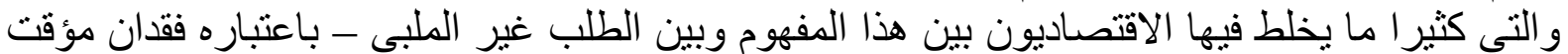

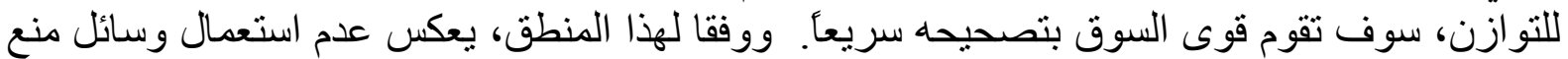

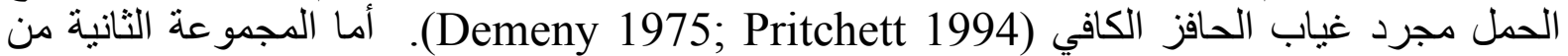
Pritchett ) patronizing النقد، فهي تقول أن مفهوم الاحتياجات غير الملباة له طابع التفضل الأبوي

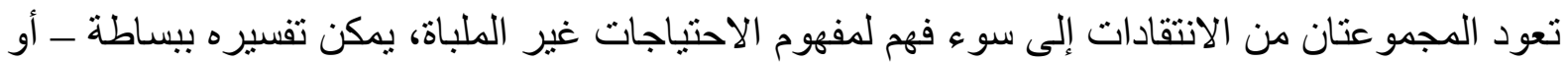

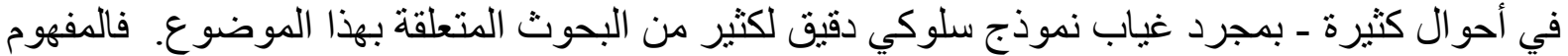

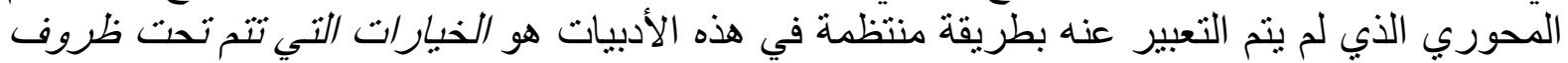

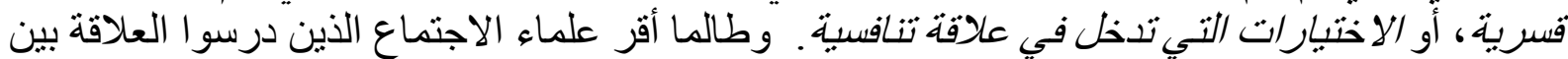

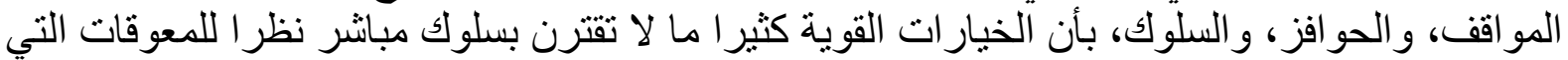

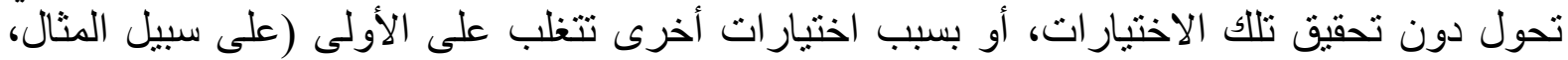
يمكن النظر في مر اجعات كل من: : Ajzen 1993; Eagly and Chaiken 1998; Dawes 1998

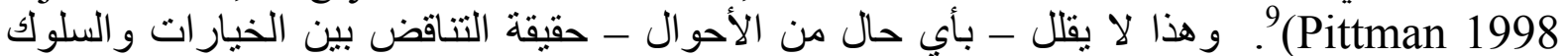

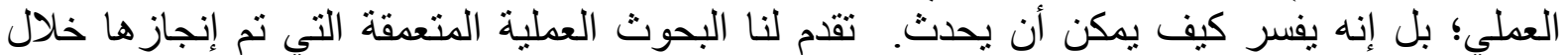

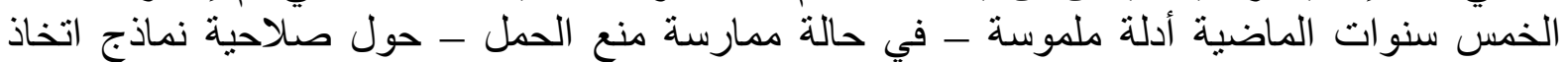

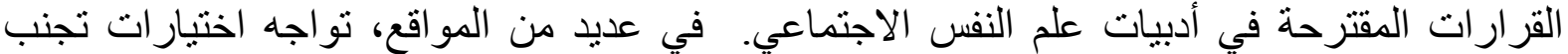

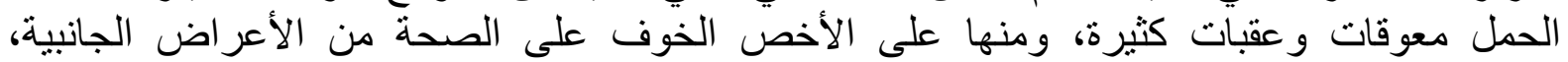

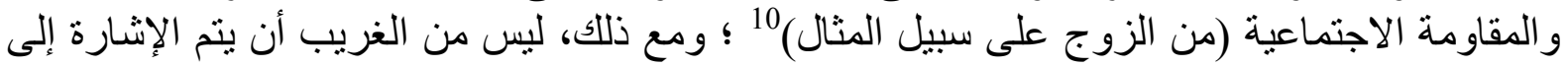


نسبة مهمة من الو لادات باعتبار ها غير مرغوب فيها. يصف ستاش (Stash 1999) الوزن المدروس

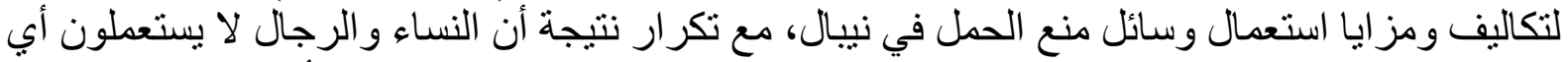

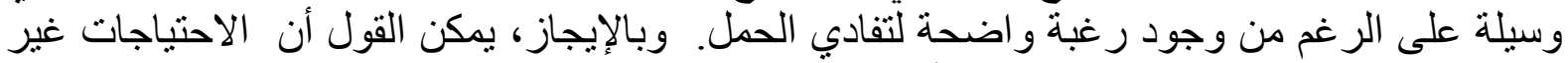

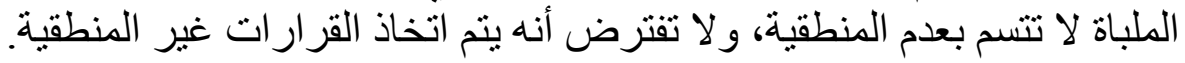

\section{الاحتياجات غير الملباة والانتقال في معدلات الخصوبة}

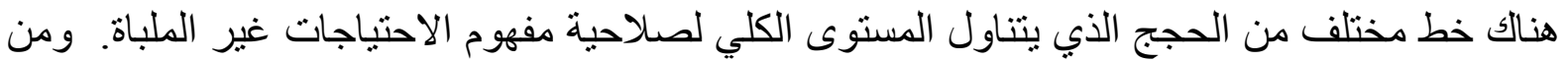

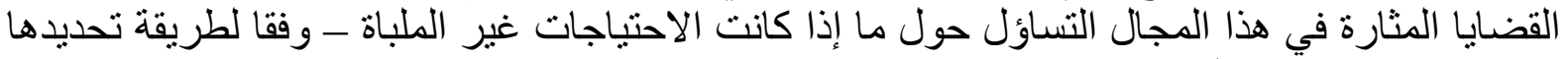

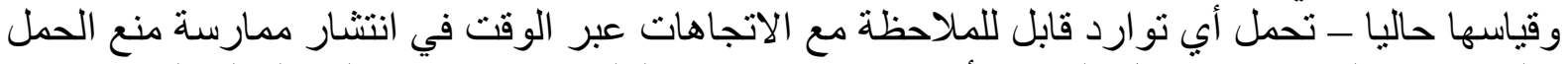

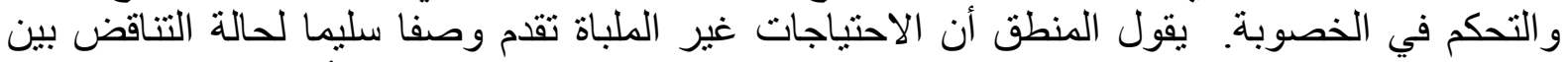

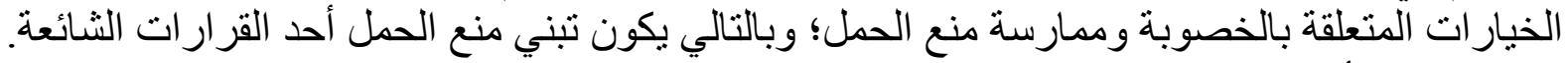

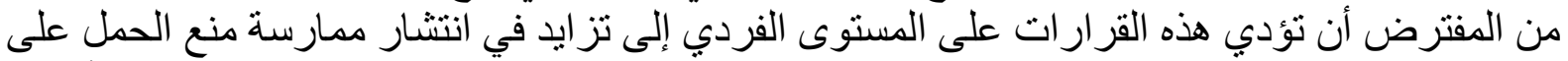

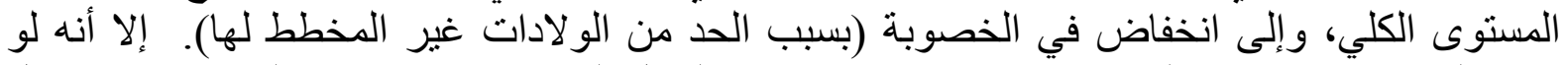

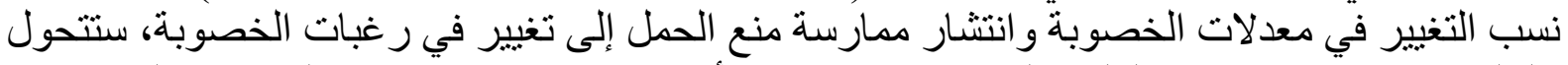

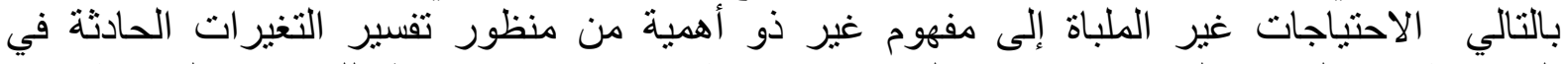
الخصوبة عبر الوقت و الفضاء، وسيكون المفهوم ذو منفعة محدودة كمفهوم منظم للسياسات السكانية السية.

يتبنى بريتشت (Pritchett 1994) هذا الهجوم في مقال مؤثر 11؛ بالإشارة إلى الارتباط الوثيق بين

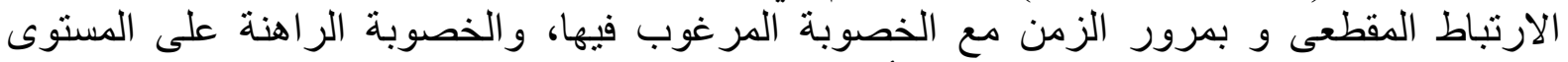

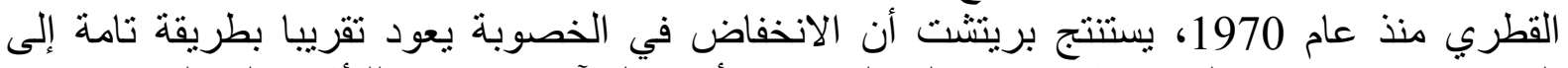

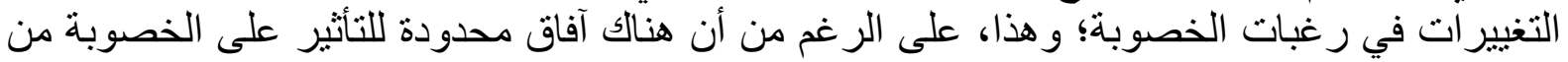

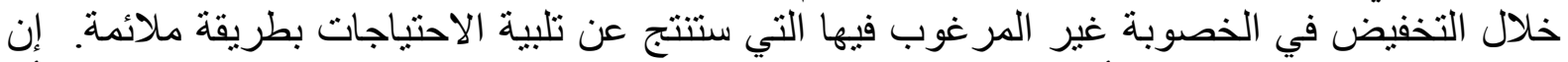

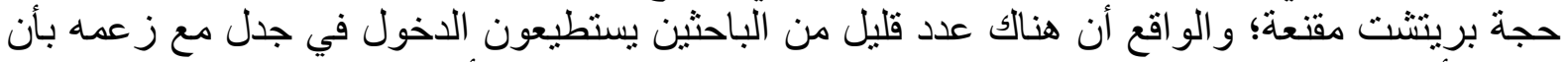

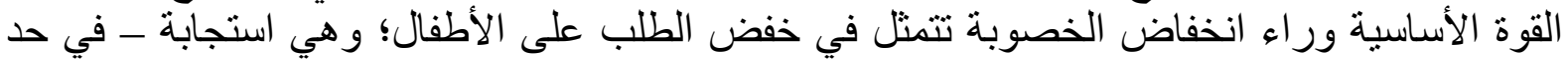

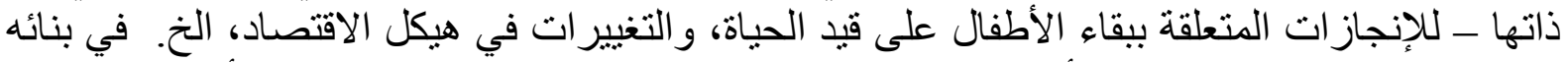

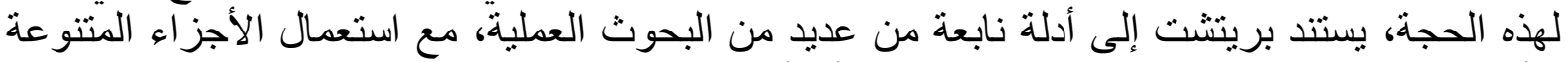

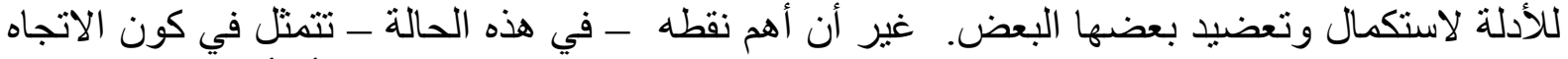

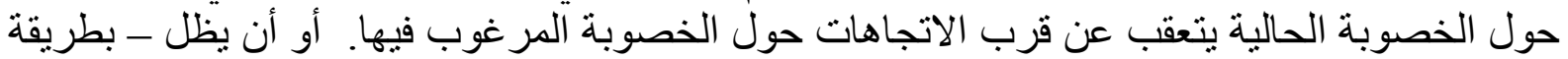
مماثلة - مستوى الخصوبة غير المرغوب فيها مستقر نسبيا خلال مرحلة الانتقال في معدلات الخصوبة الخبة.

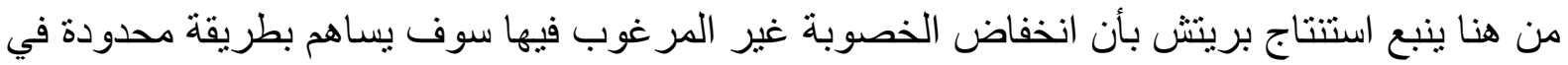

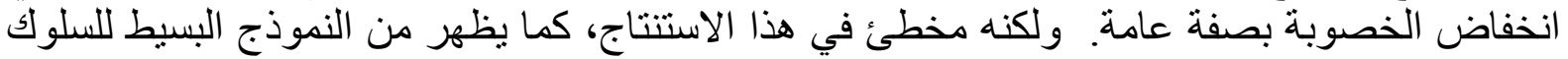

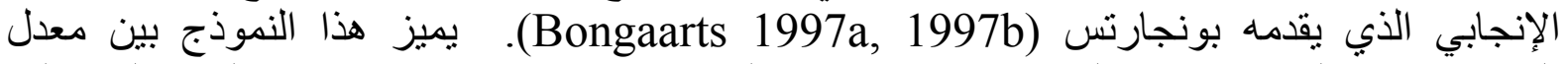

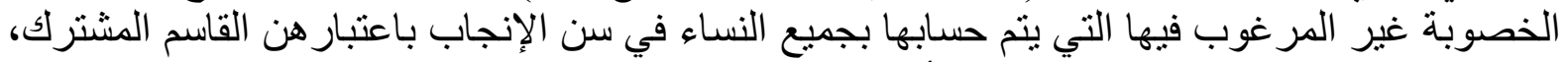

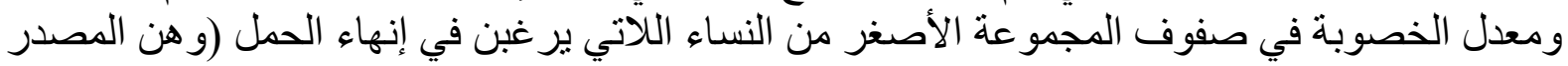

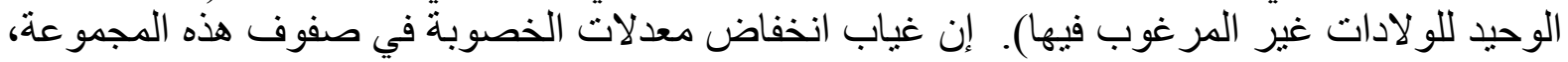

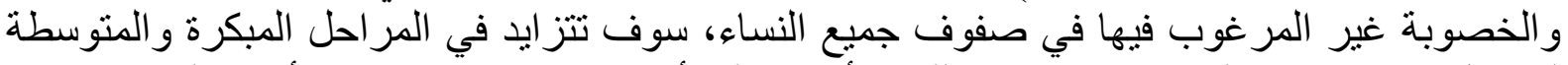

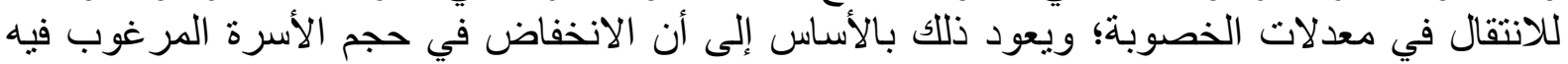

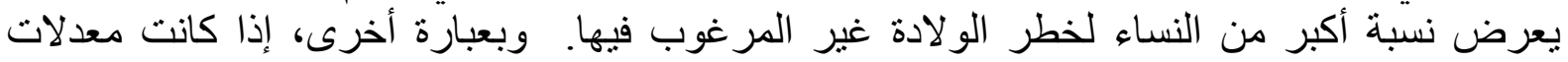

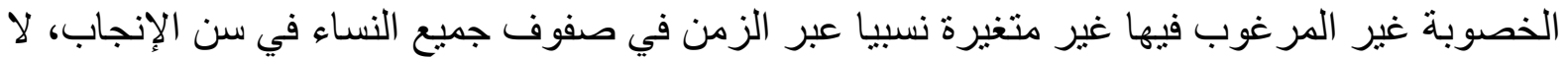

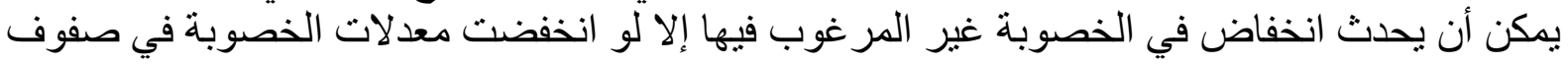

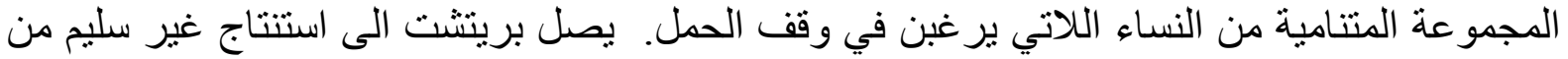




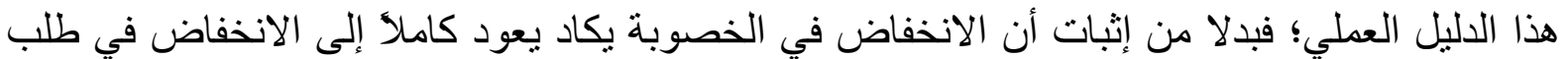

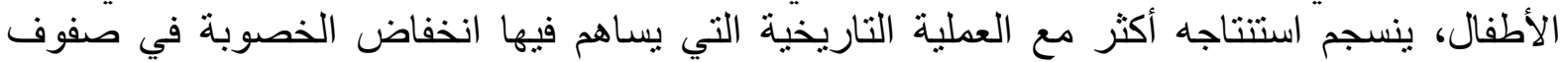

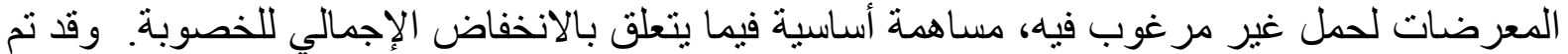

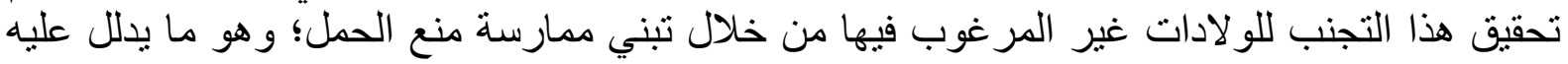
بطريقة واضحة قسم كبير من بيانات المسوح.

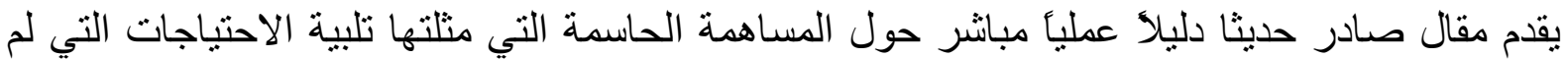

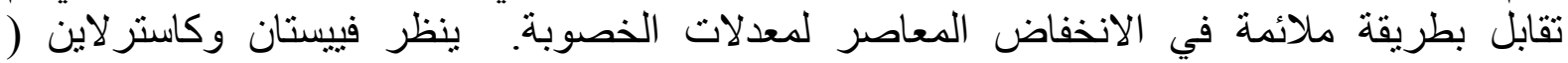

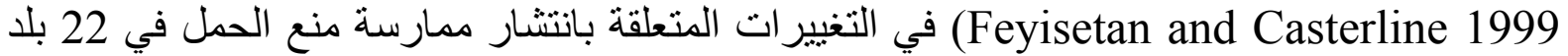

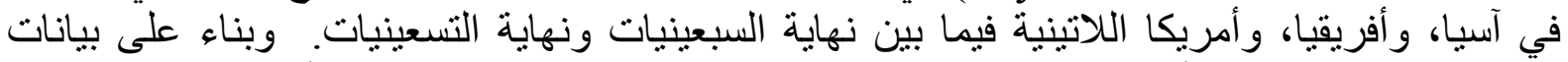

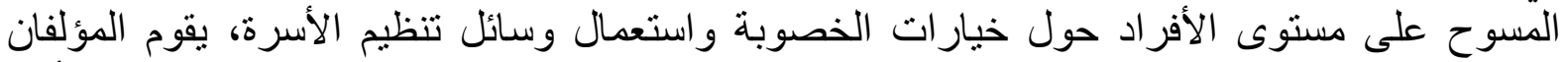

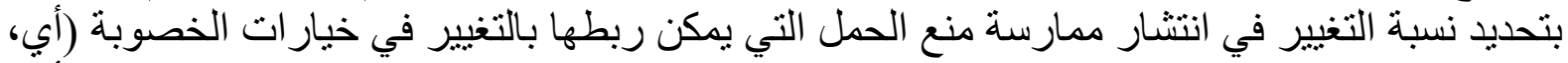

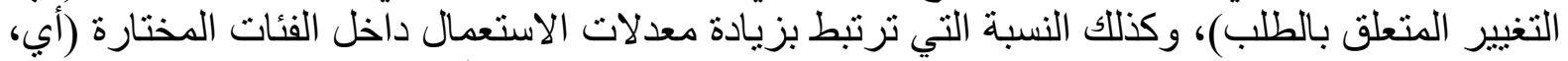

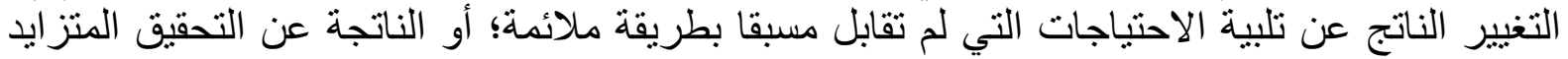

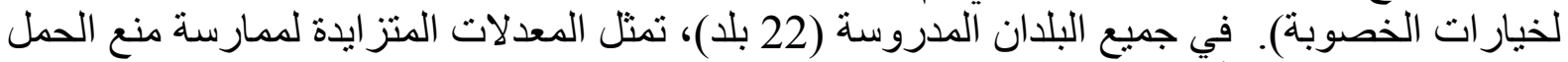

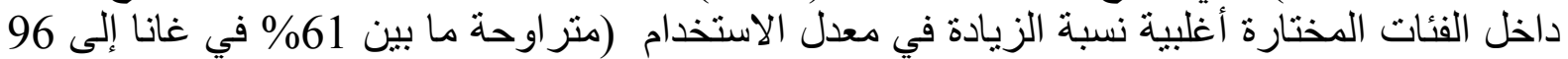

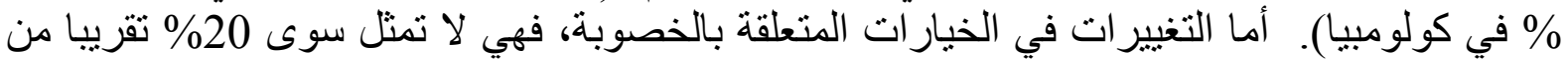

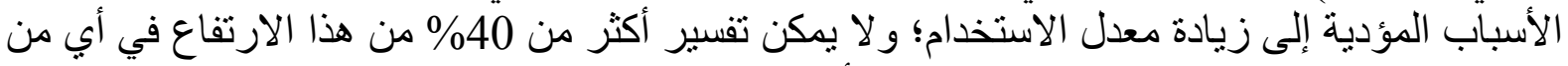

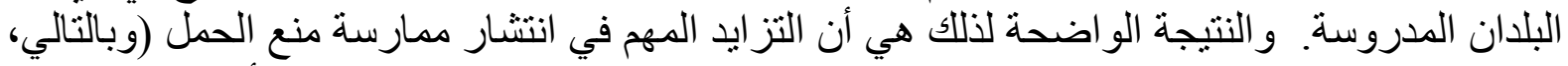

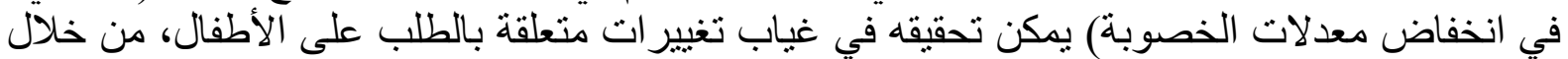

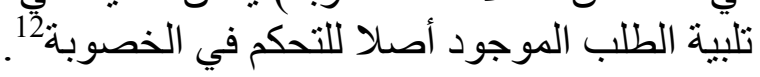

تضفي هذه الدراسة الصلاحية على مفهوم الاحتياجات غير الملباة، وتمثل المقابل على المستوى الكلي

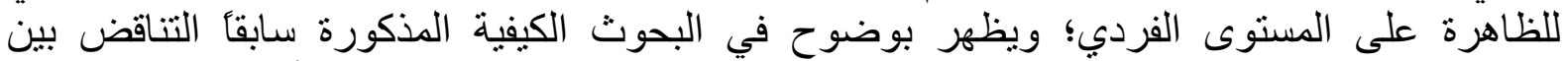

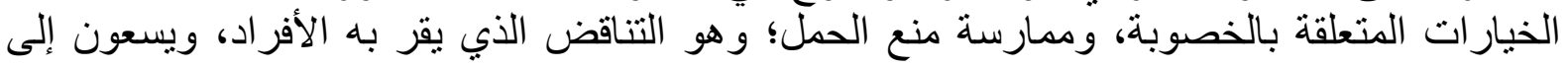

\section{الاحتياجات غير الملباة والطلب على تنظيم الأسرة}

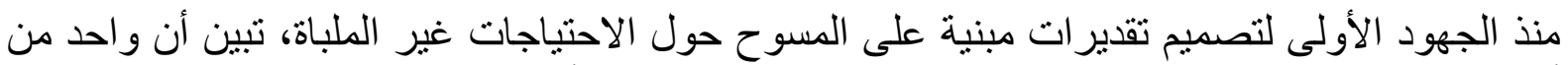

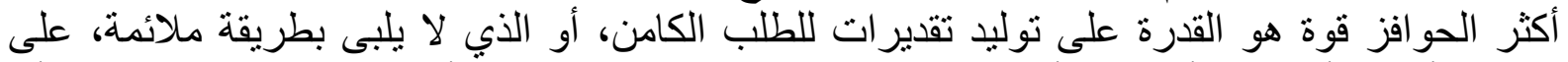

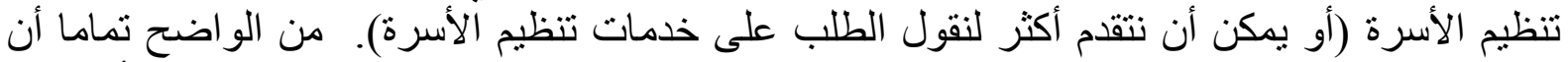

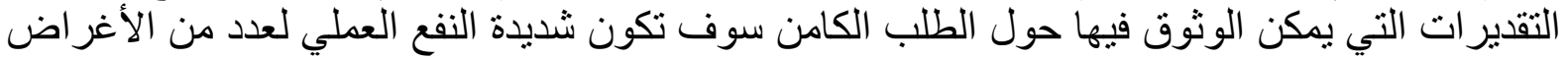

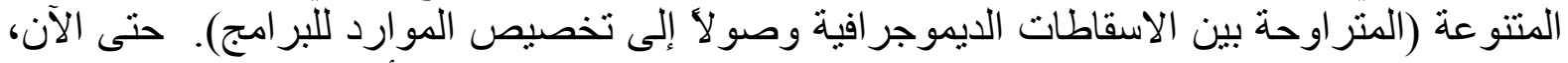

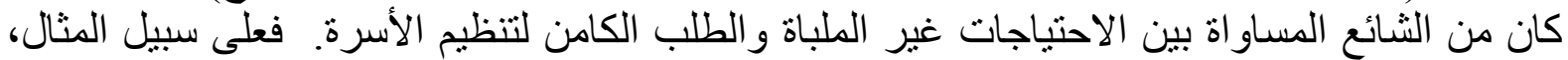

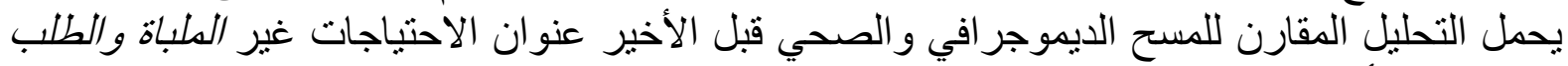

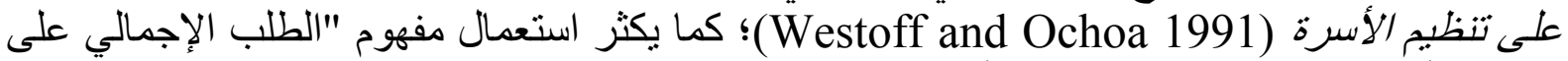

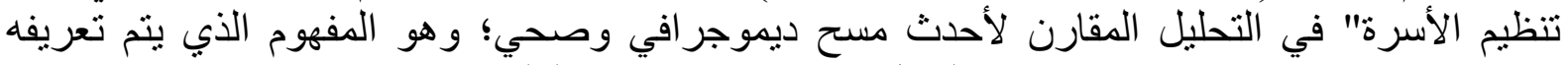

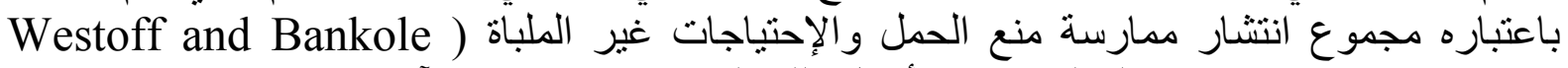

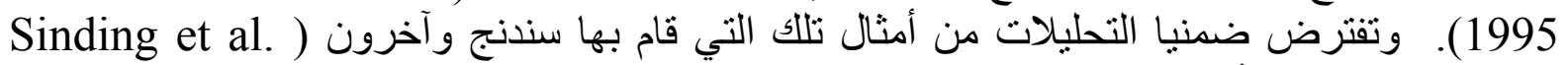

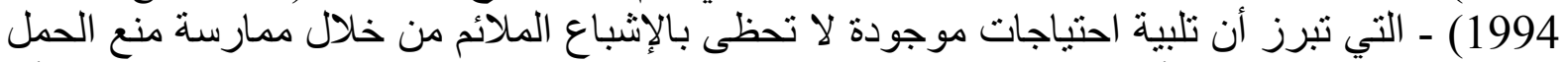

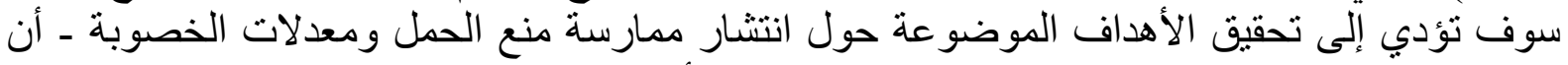
الاحتياجات غير الملباة تساوي الطلب الكامن على تنظيم الأسرة. 


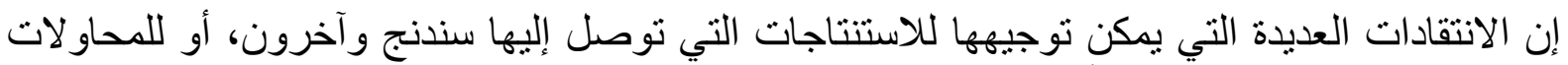

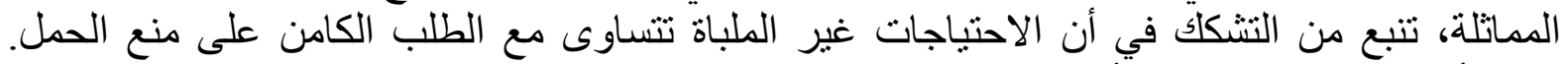

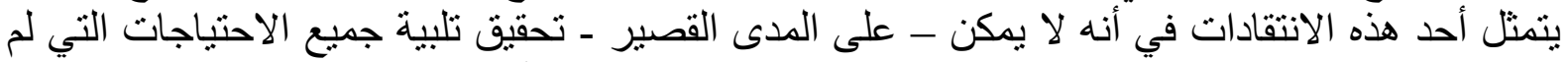

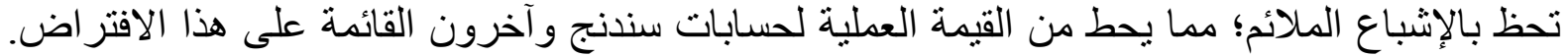

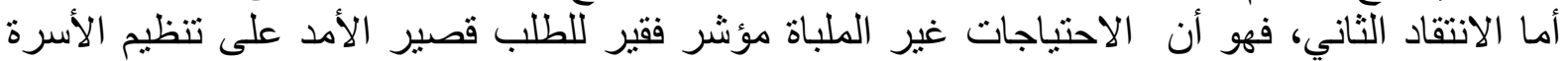
(وخاصة على خدمات تنظيم الأسرة) مقارنة بالمؤشرات الماتئ المتو افرة الأخرى. وسوف نتناول الانتقادين

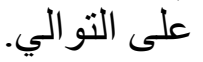

بدءا بالانتقاد الأول، ليس هناك شك بأنه من غير المرجح أن تقوم عديد من النساء لا تلبى احتياجاتهن

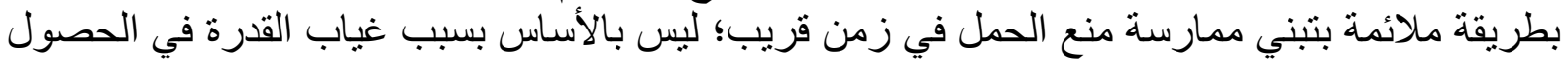

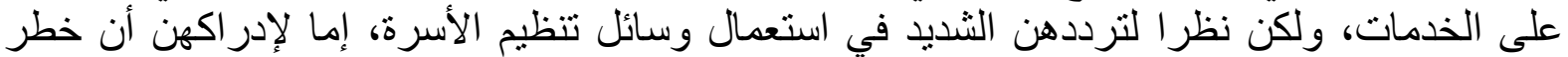
تعرضهن للإنجاب منخفض، أو لأسباب اجتماعية، أو ثقافية، أو صحية أخرى (Pritchett 1994).

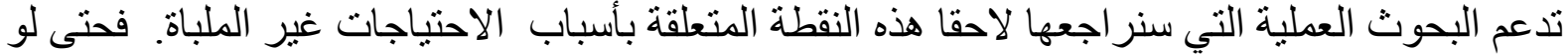

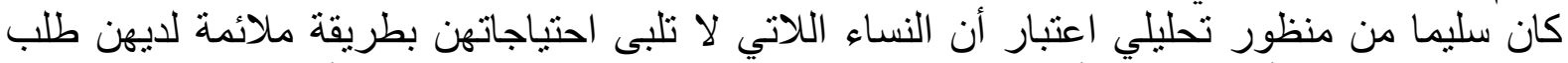

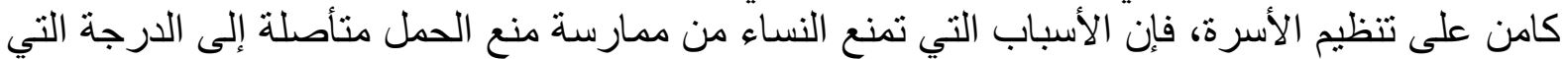

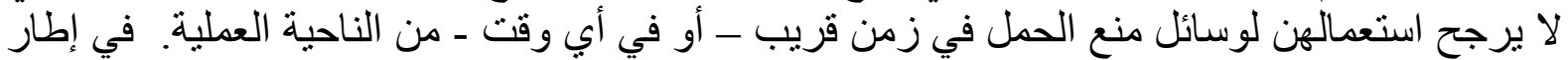

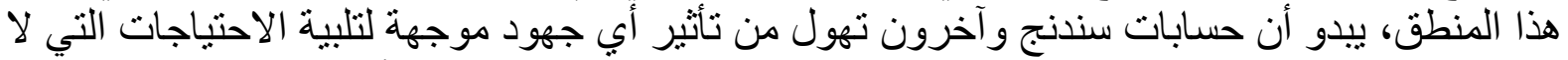

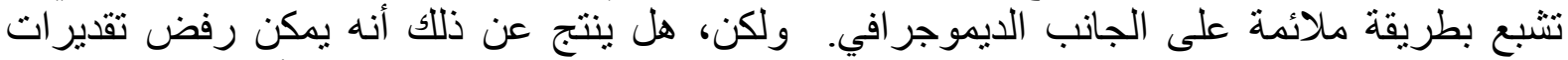

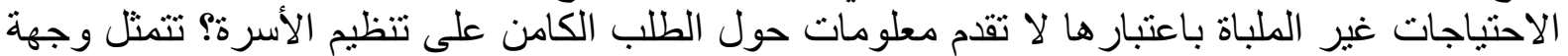

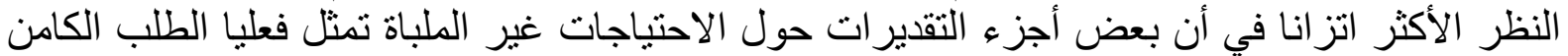
على تتظيم الأسرة الذي يمكن أن يتحول إلى استعمال وسائل منع الحمل.

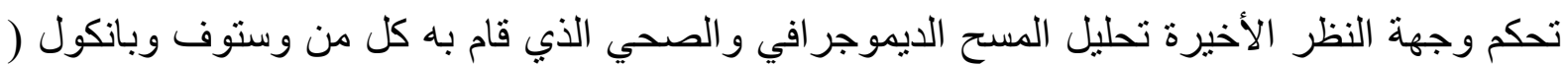
(Westoff and Bankole 1996

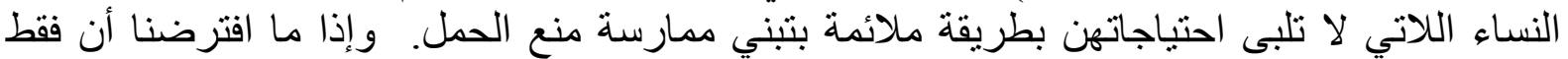

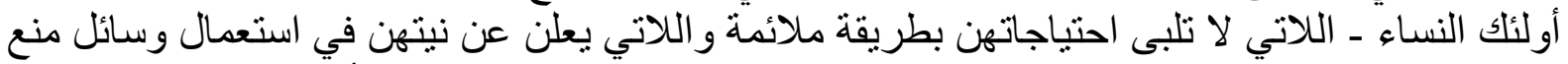

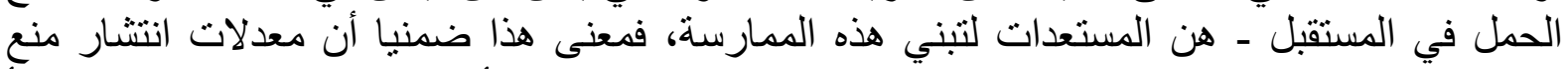

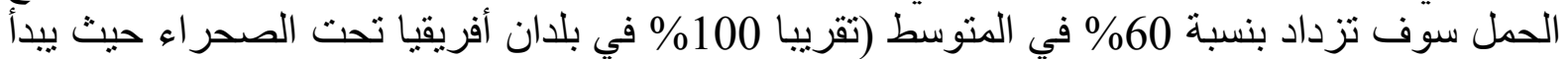

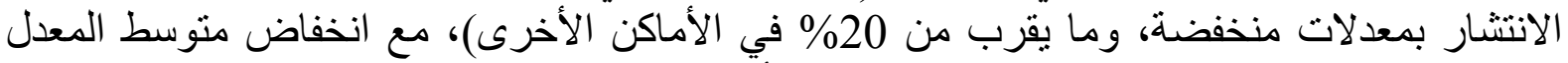

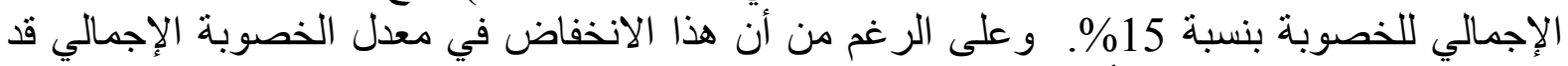
يبدو بسيطا، فهو يمثل في أغلبية البلدان من 20\% الرئ من إلى 50\% من المسافة إلى مسنوى الاحلال

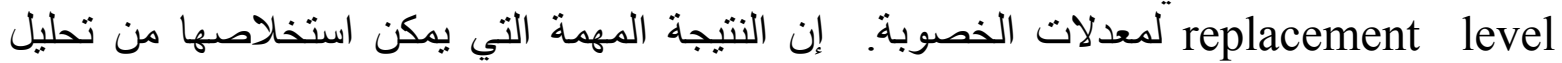

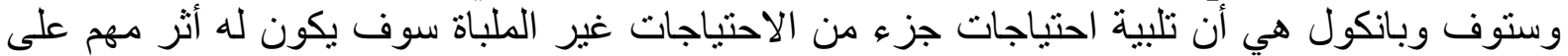

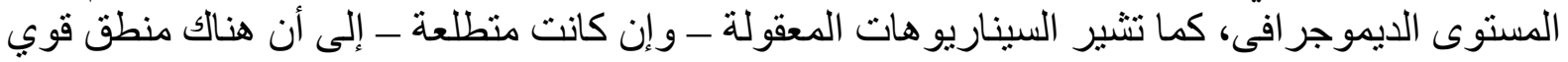
من الناحية الديموجر افية للتركيز على الاحتياجات غير الملباة.

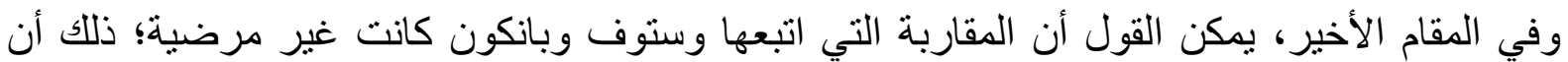

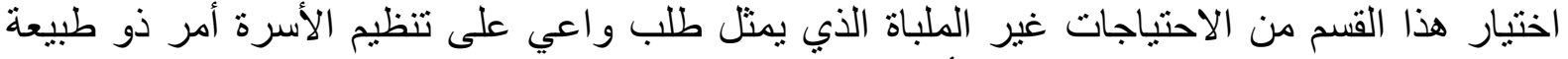

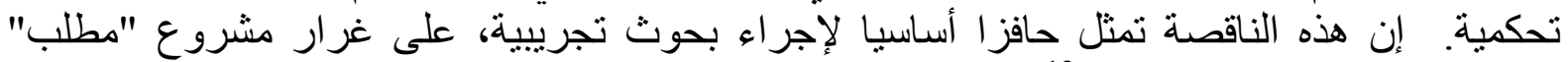
(Matlab)

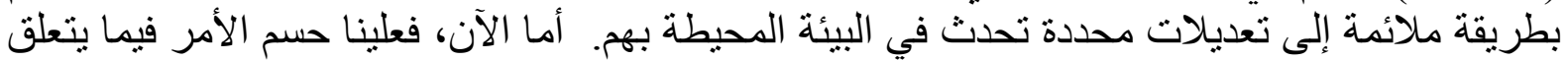

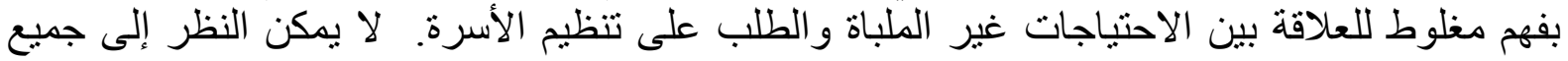




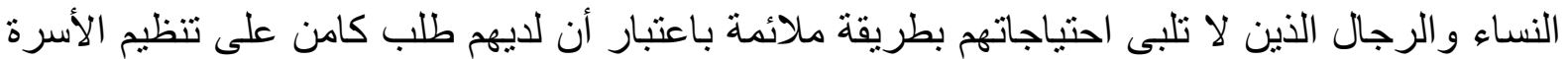

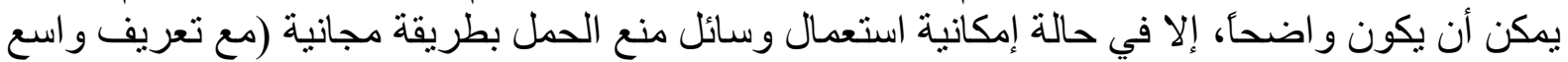

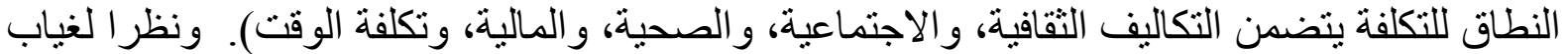

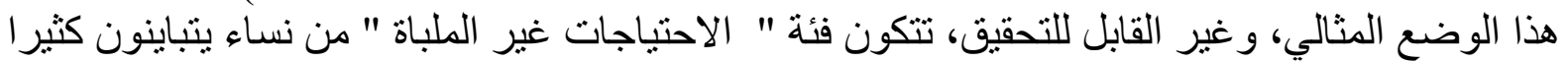

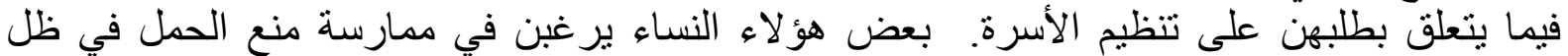

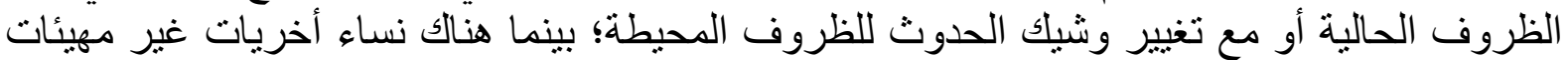

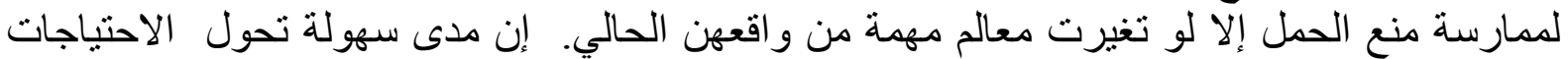

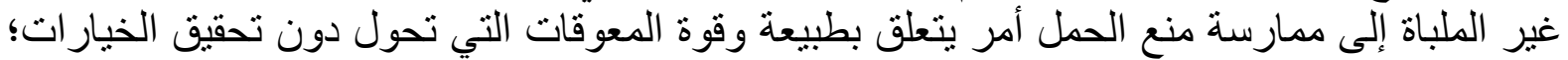

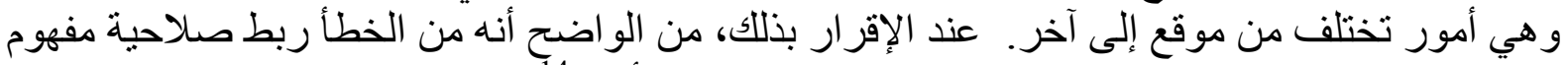

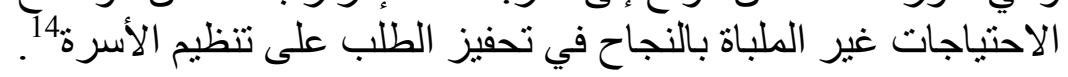

يؤدي بنا هذا إلى الانتقاد الثاني المتعلق باستعمال الاحتباجات غير الملباة كمؤشر للطلب على ممارسة

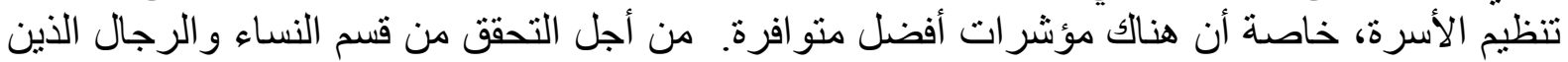

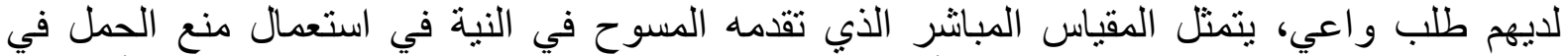

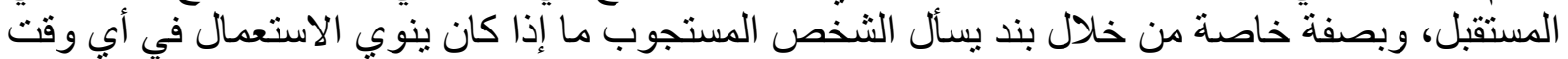

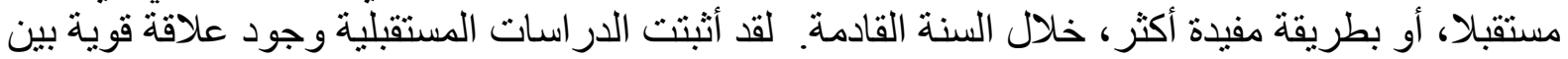
النية في الاستعمال والسلوك اللاحق فيما يتعلق بمنع الحمل (على سبيل المثال:. ;

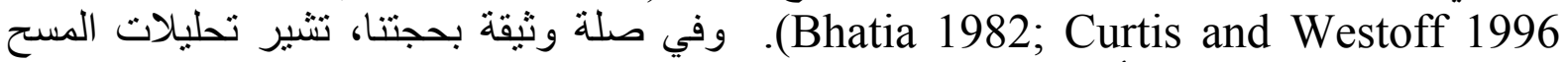

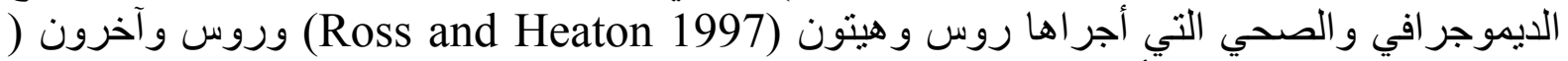

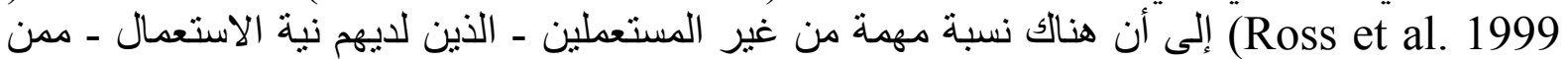

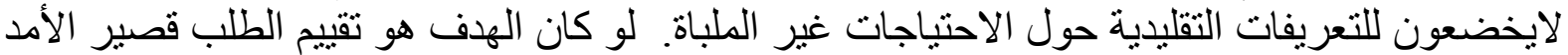

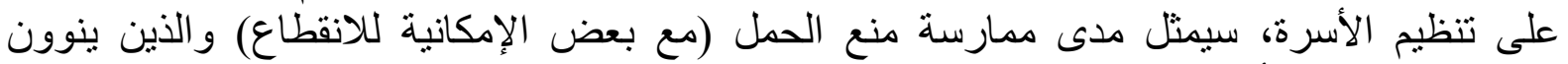

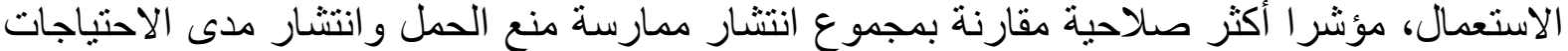

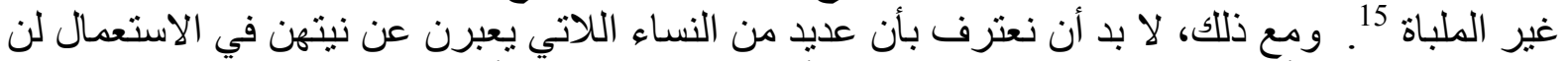
يقمن ذلك، على الأقل في المستقبل القريب، نظر الأنواع الكاع المعوقات التي أثرنا إليها سابقا.

\section{توسيع المفهوم}

تتمثل واحدة من الانتقادات التي تساق بطريقة متنامية حول مفهوم الاحتياجات غير الملباة في أن الدفهوم

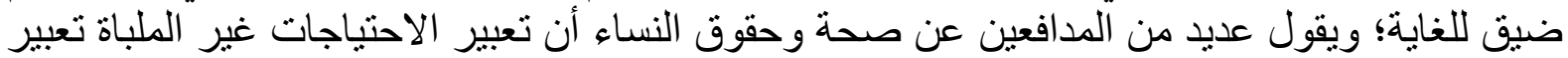

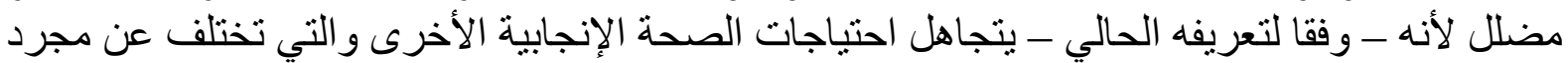

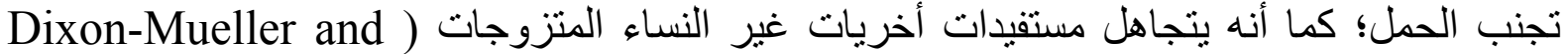
1993

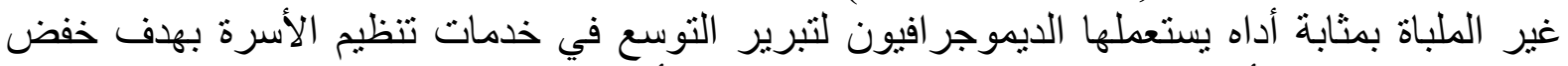

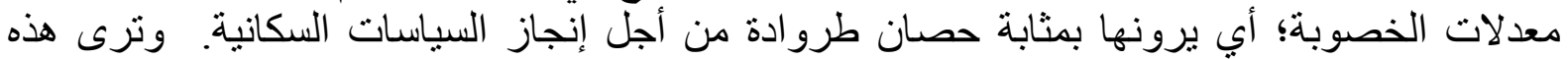

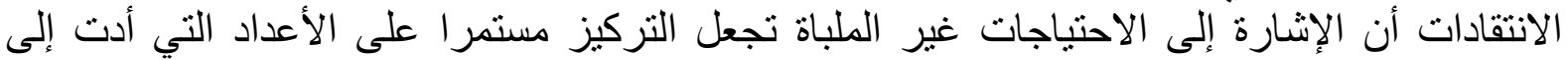

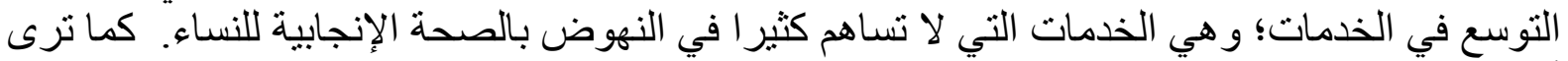

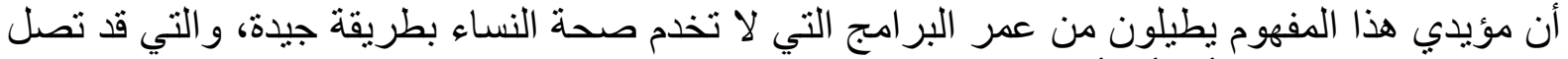

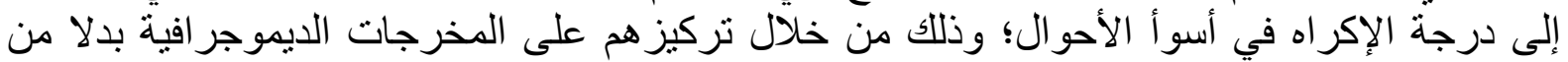

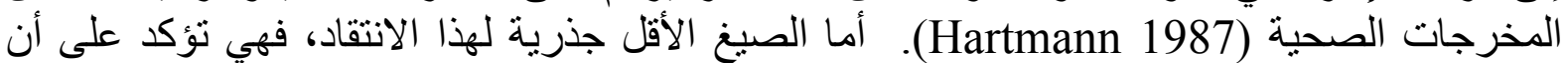

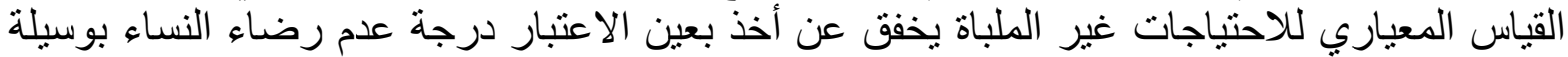

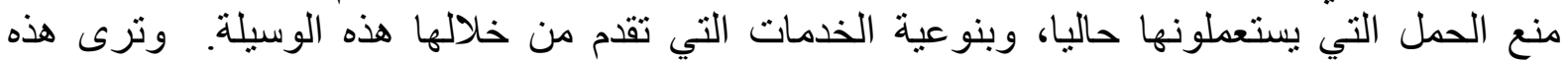

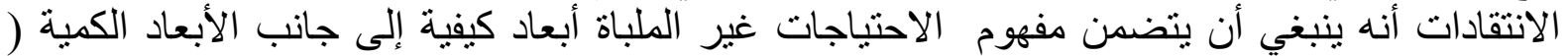




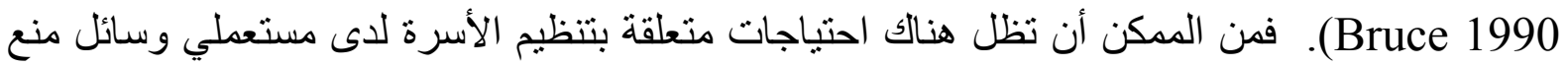

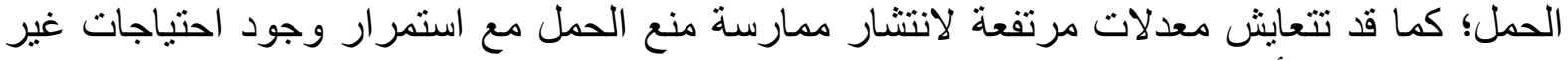

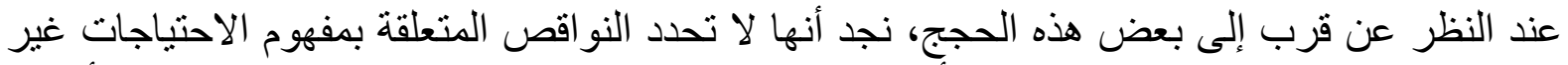

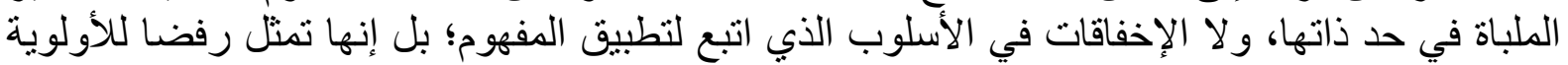

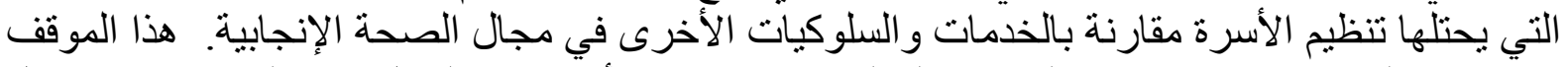

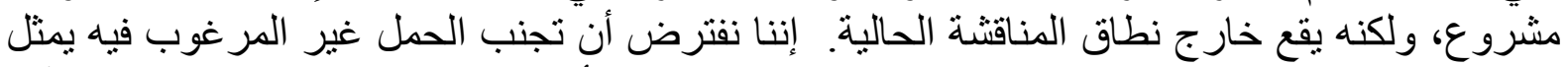

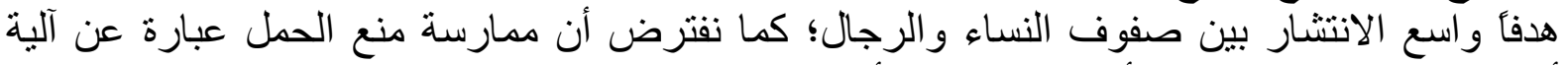

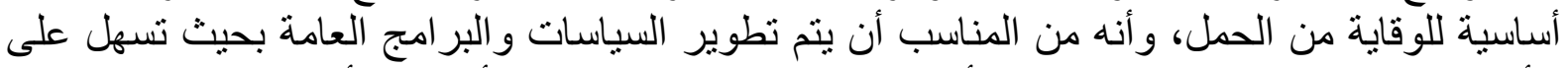

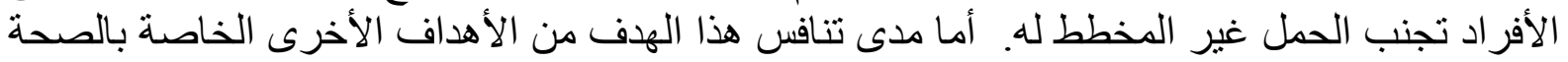

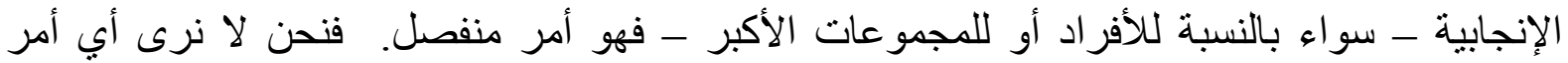

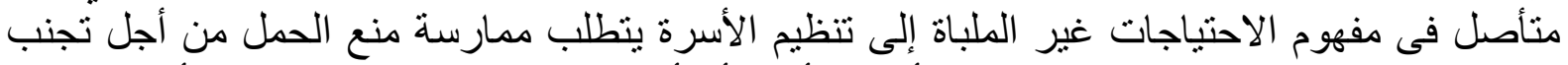

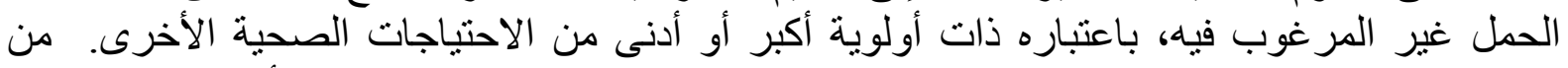

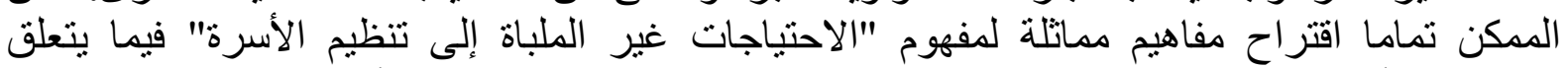

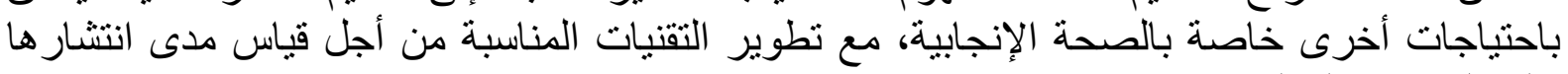
على المستوى العملي (Omran et al. 1992; Short 1994).

أما فيما يتعدى تلك القضايا الفلسفية الأوسع، فندن نعتبر أن هناك قيمة عملية كبيرة في تبني مفهوم يركز

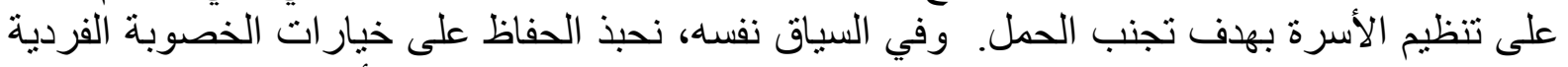

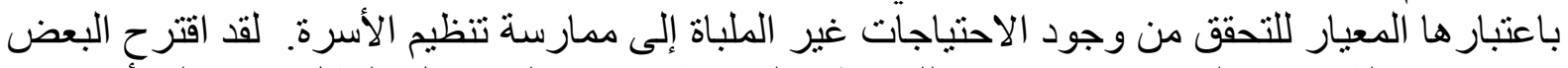

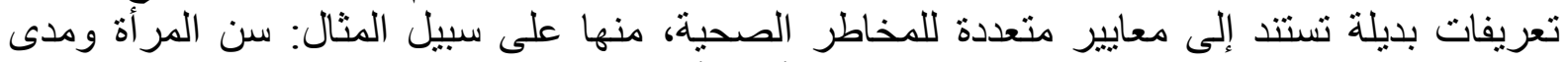

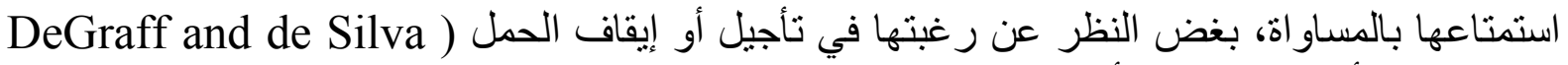

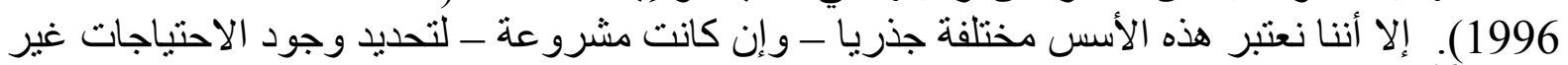

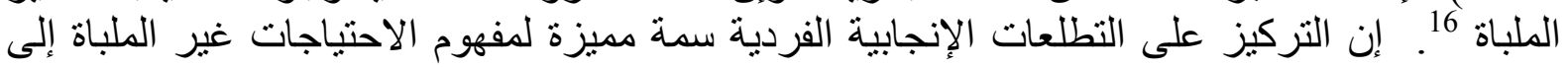
تنظيم الأسرة الذي برز منذ عدة عقود.

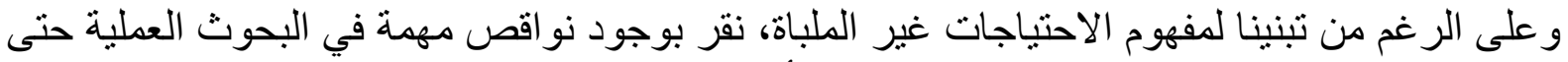

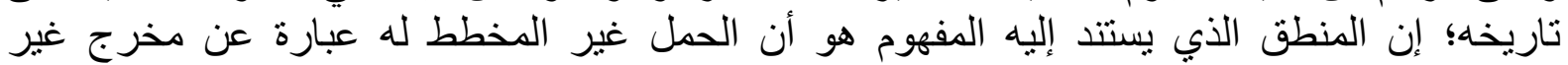

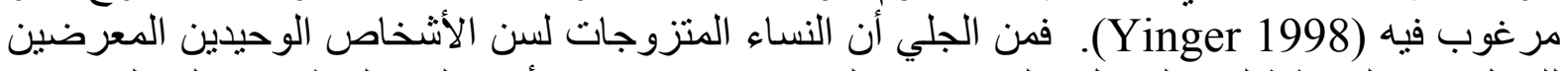

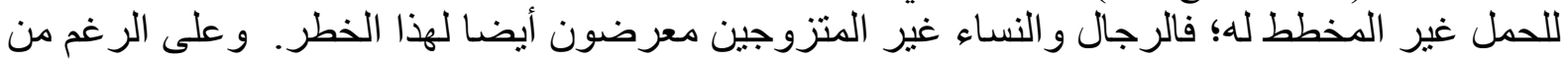

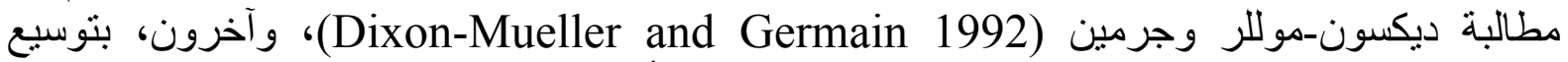
تعريفات الاحتياجات غير الملباة بحيث تمند إلى مجموعات أخرى، فقد كان التقدم في هذا الاتجاه مخيبا

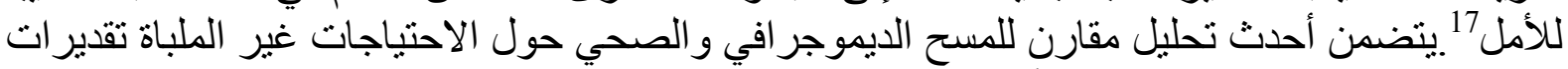

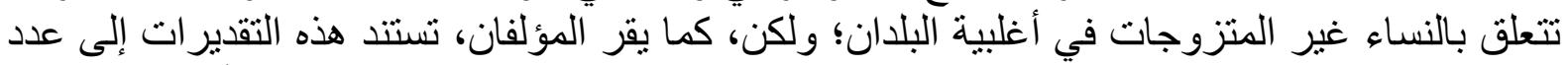

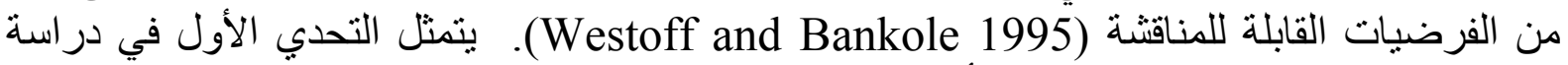

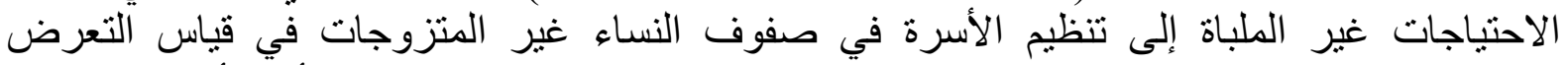

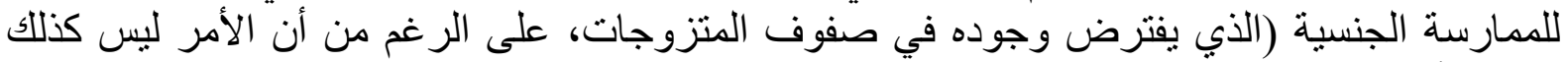

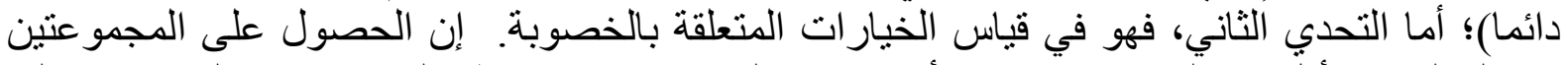

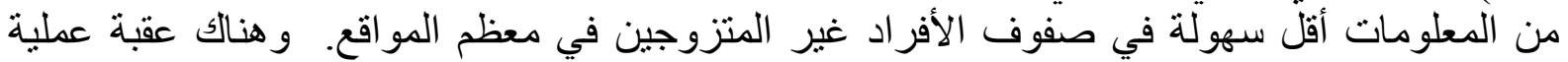

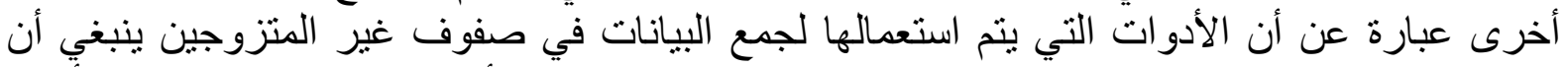

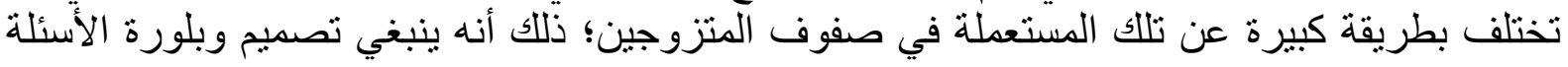


المتعلقة بر غبات الخصوبة، وخبر ات الحمل والإنجاب، و المعرفة بمنع الحمل وممارسته، بحيث تتناسب مع ظروف حياتهم المختلفة.

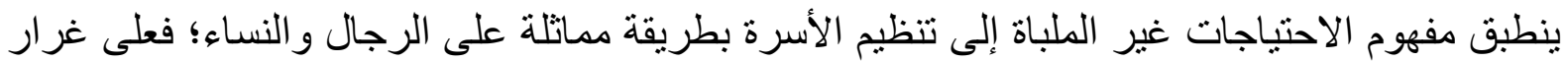

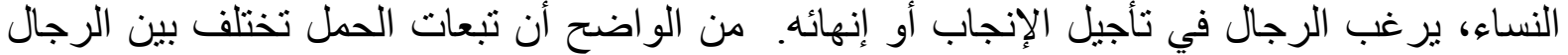

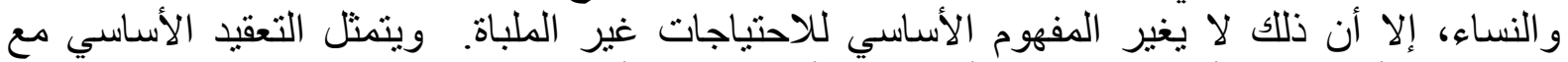

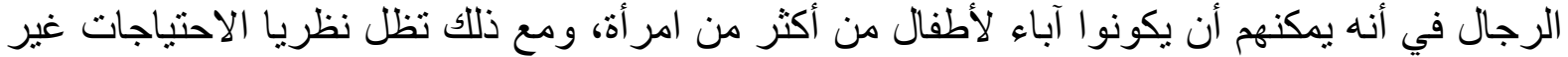

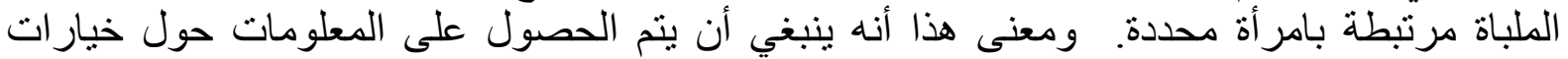

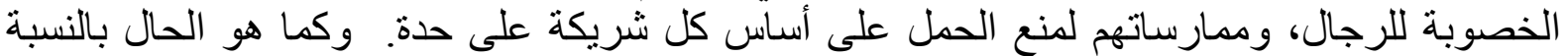

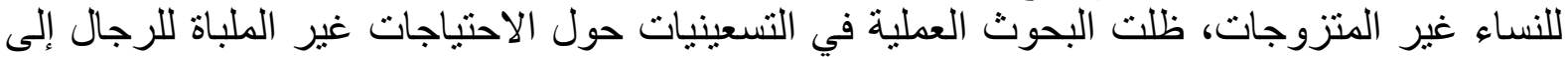

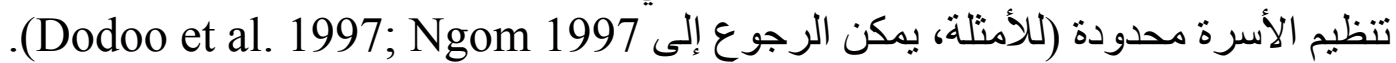

كما أن هناك مجالأ بحتئ مرتبطأ بهذا الأمر وهو " الاحتياجات غير الملباة للزوجين" ( Bankole and

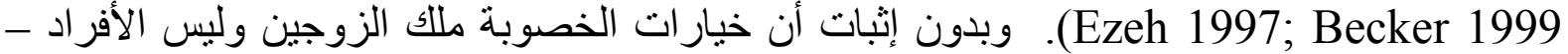

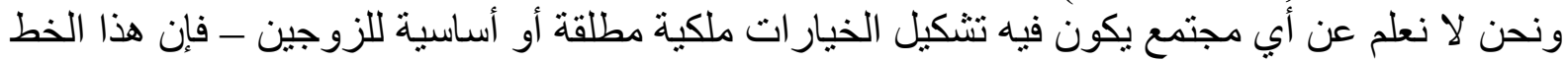

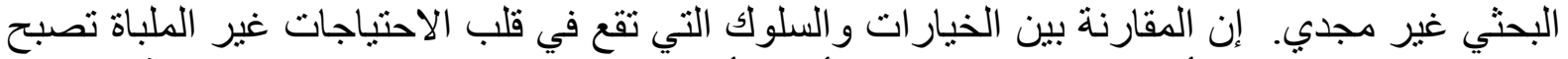

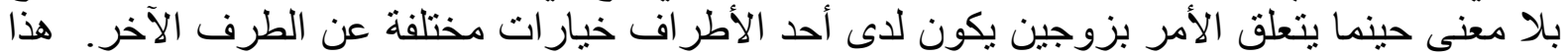

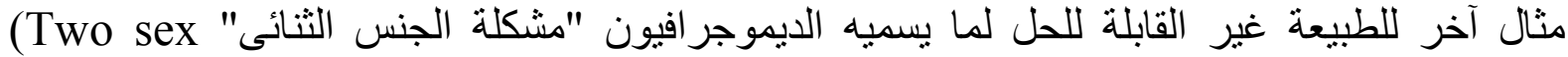

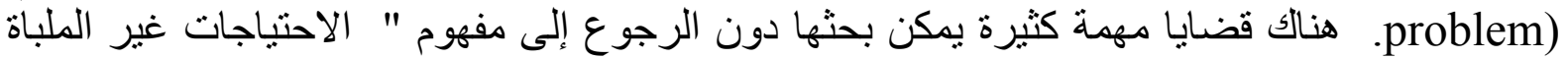

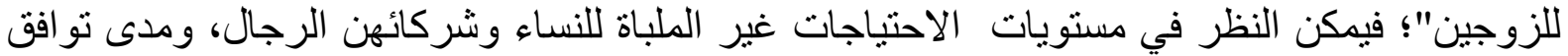

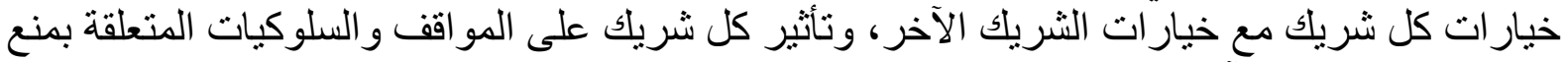
الحمل على الثريك الآخر (Ezeh 1993).

أما المجال الأخير الذي انتقدت فيه المقاربة التقليدية لدفهوم الاحتباجات غبر الملباة بالضيق، فهو تجاهل

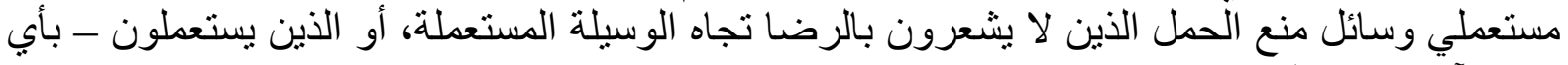

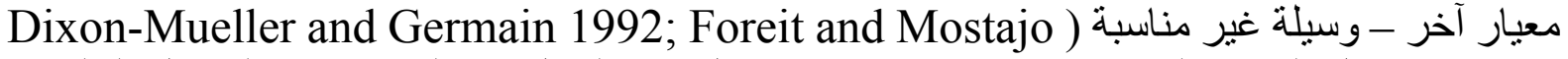

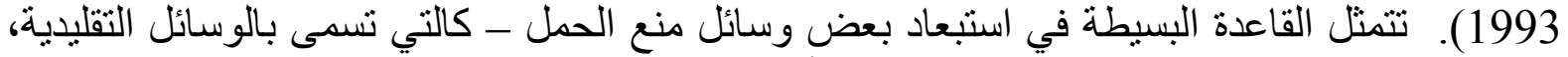

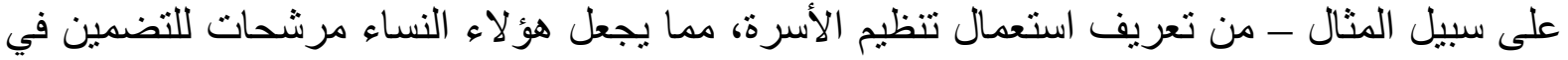

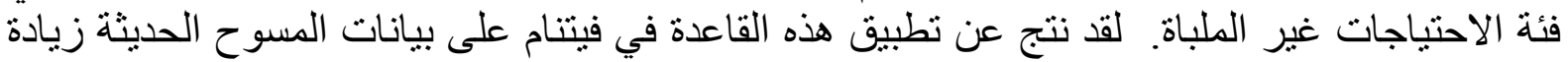

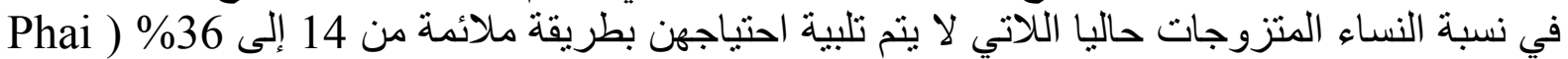

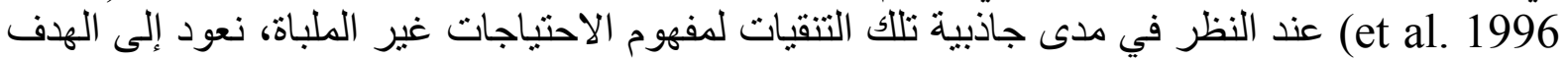

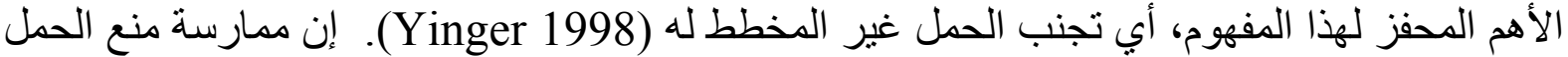

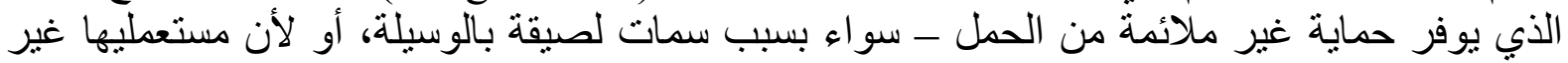

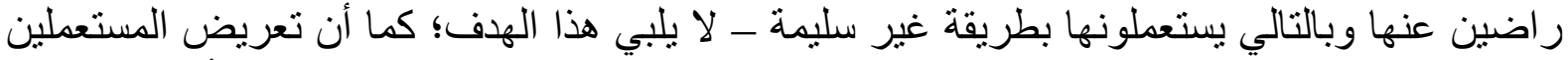

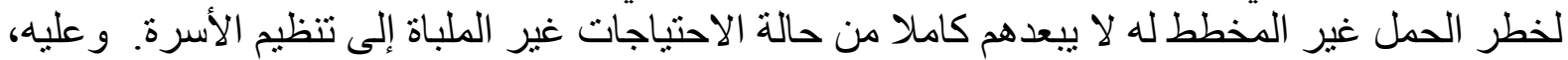

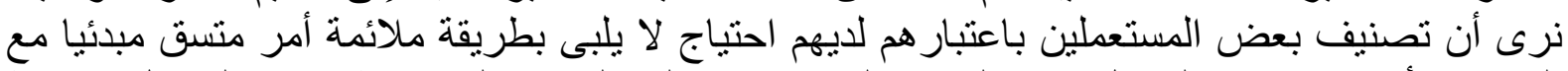

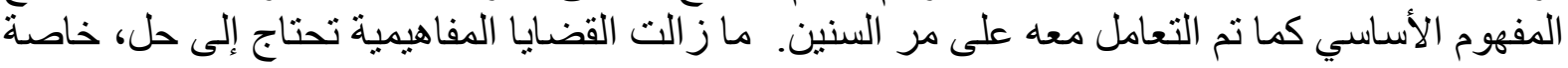

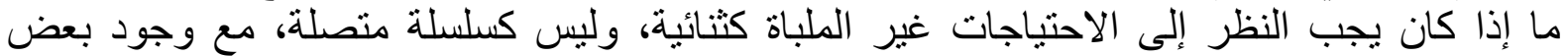

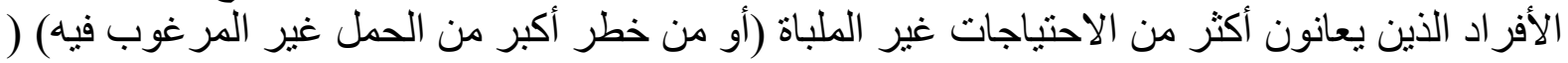
El-Zeini forthcoming

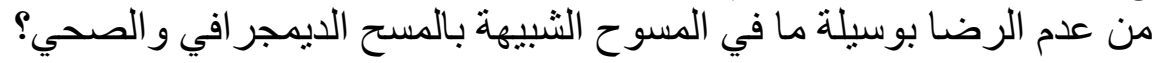




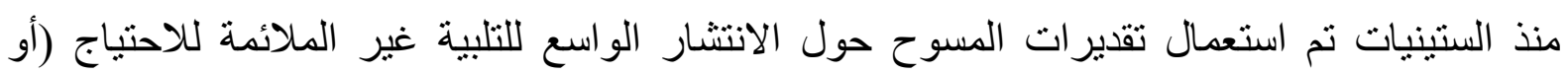

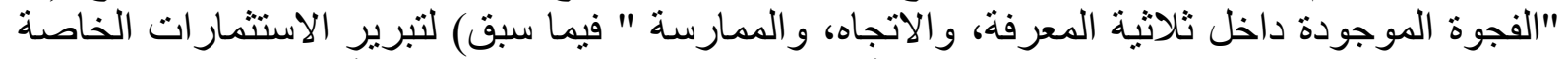

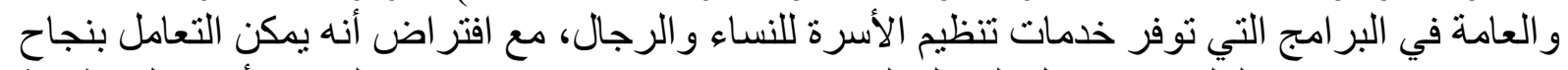

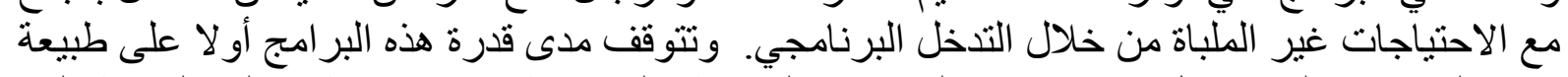

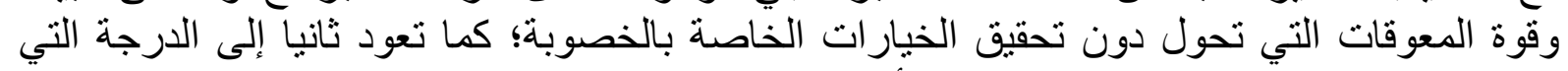

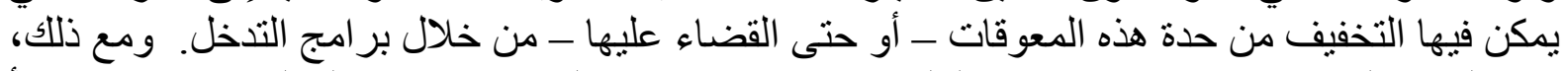

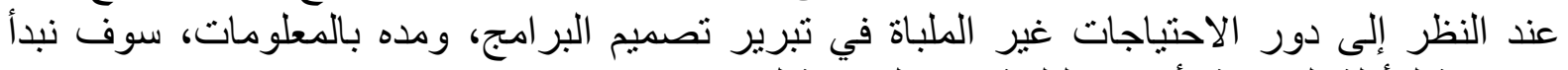
بمر اجعة للأدلة الخاصة بأسباب التلبية غير الملائمة للاحتياج.

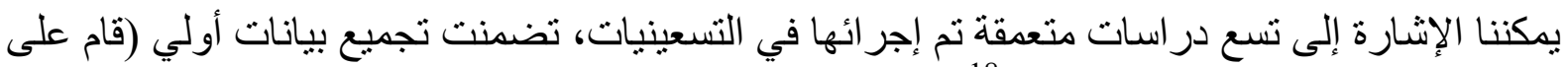

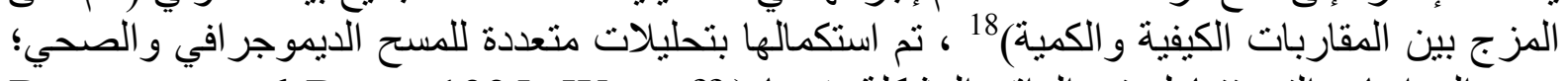

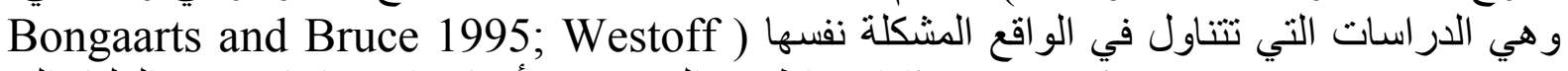

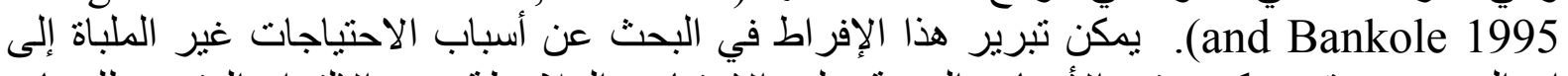

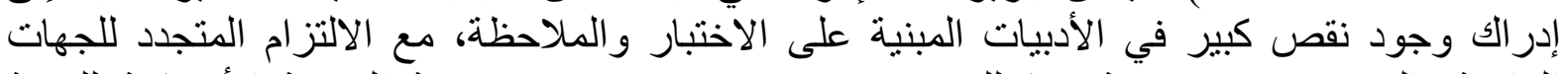

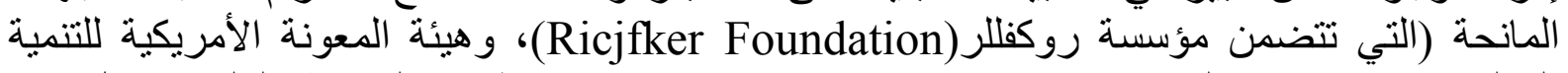

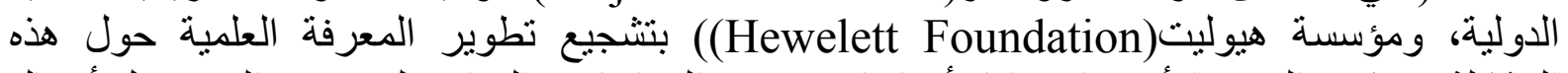

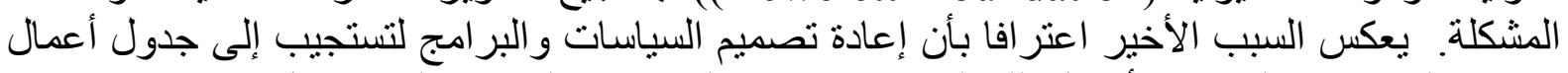

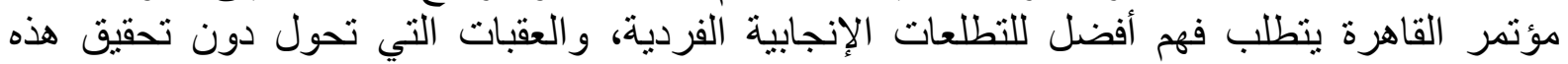

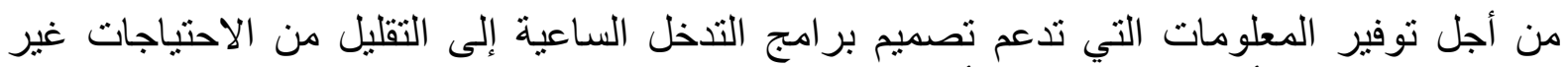

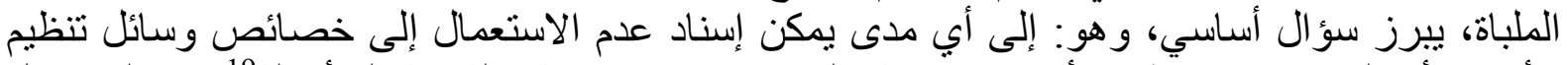

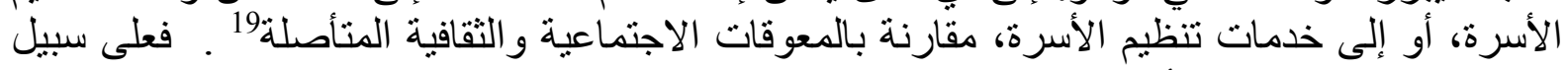

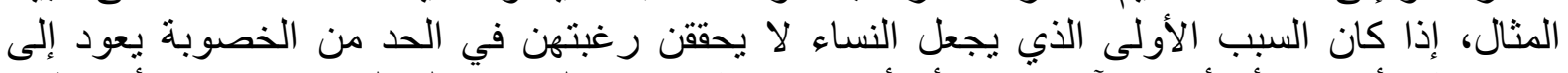

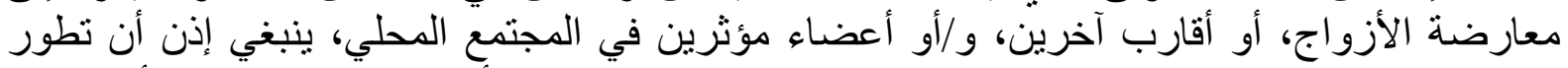

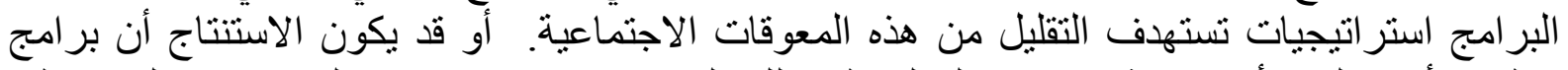

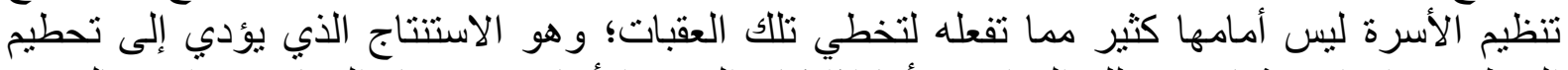

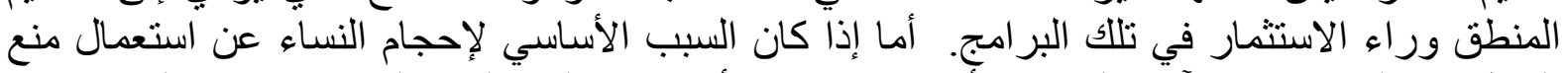

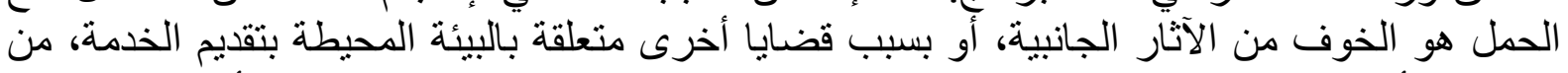

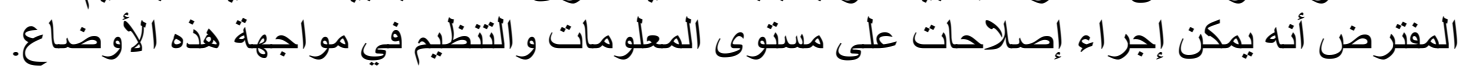

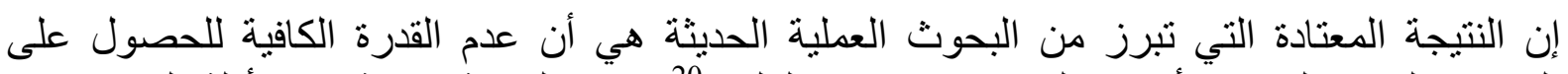

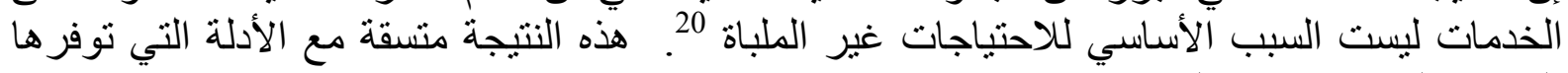

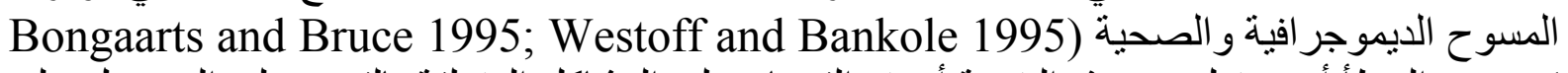

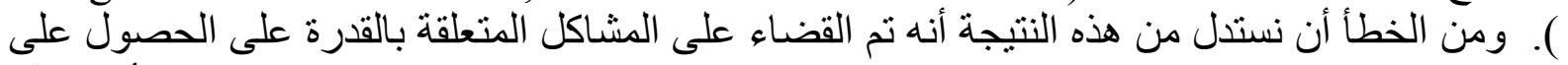

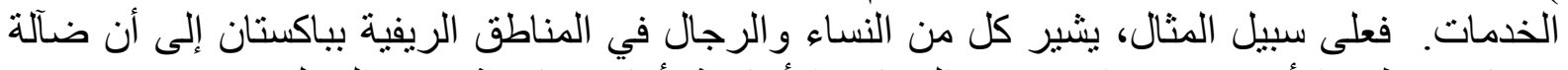

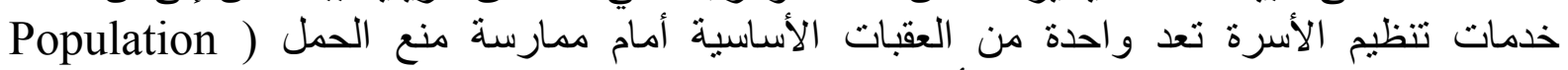

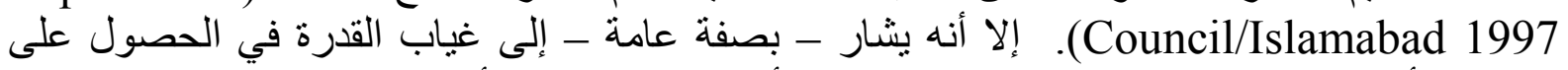

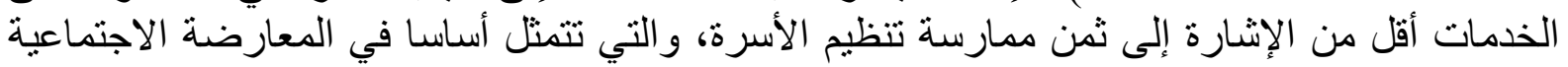

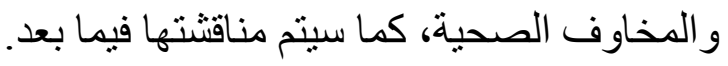


عند تقييم هذه النتيجة، ينبغي أن تبقى نقطتان حاضرتان في الذهن؛ النقطة الأولى هي أنه لا تسمح أي من

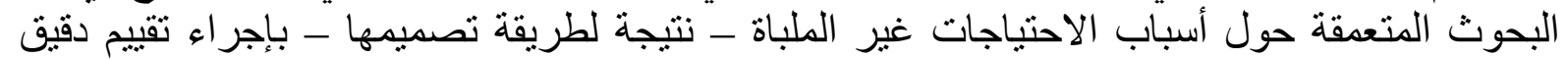

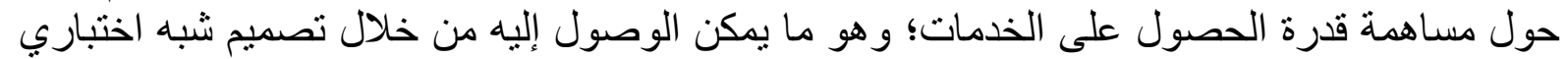

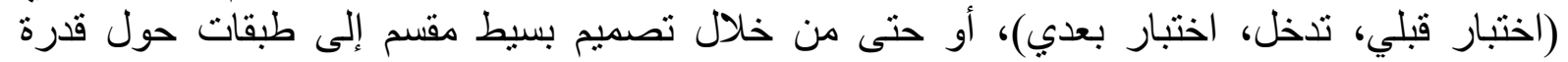

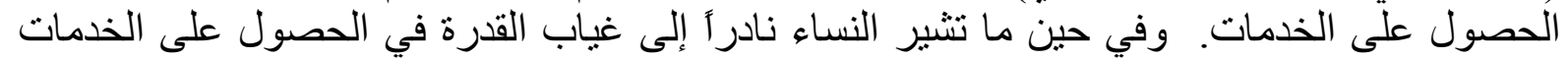

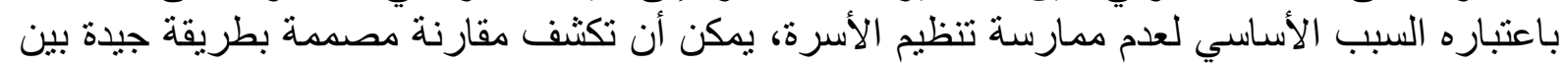

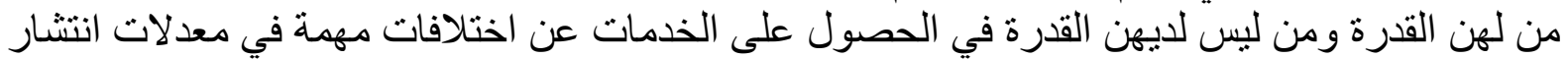

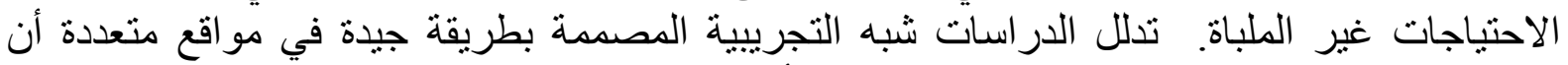

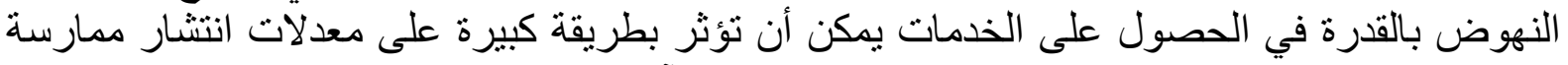

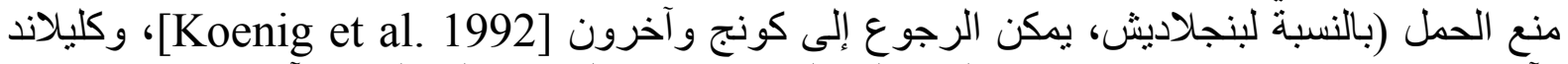

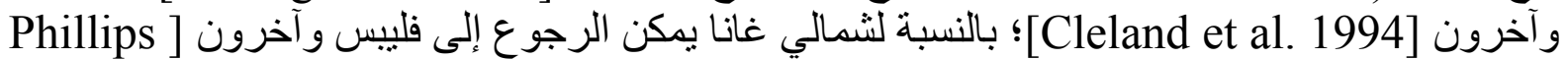

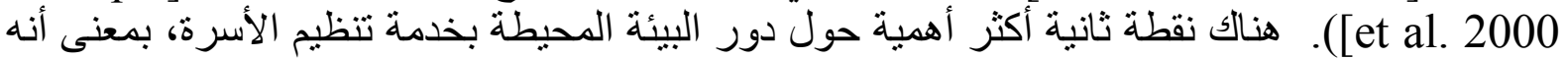

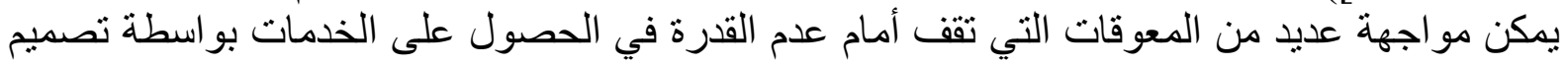
المبادر ات البرامجية المناسبة (Bongaarts and Bruce 1995).

حول أسباب الاحتياجات غير الملباة - بخلاف عدم القدرة في الحصول على الخدمة - تبرز ثلاثة أسباب

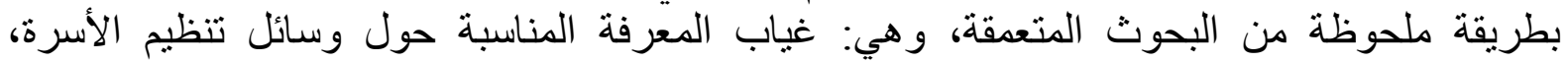
و المعارضة الاجتماعية لاستعمال تلك الوسائل، و المخاوف الصحية حول الآثار الجانبية المحتملة.

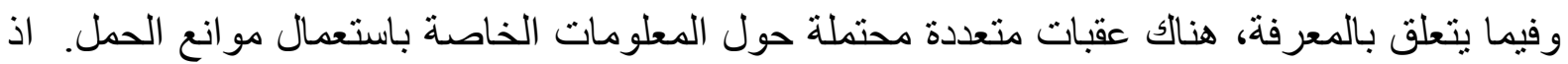

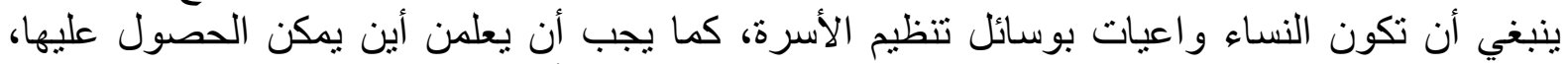

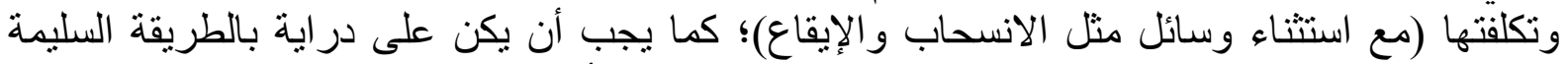

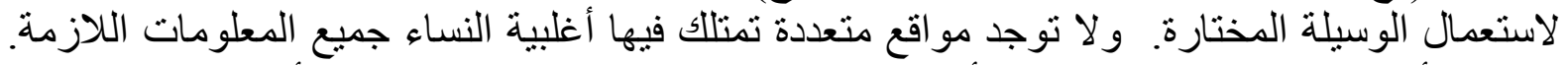

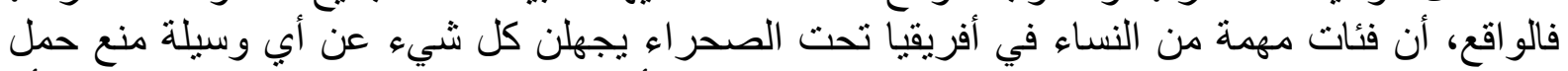

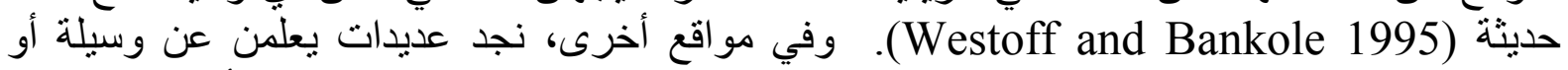

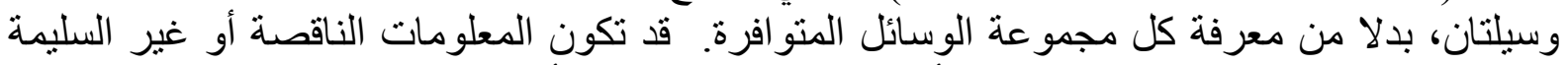

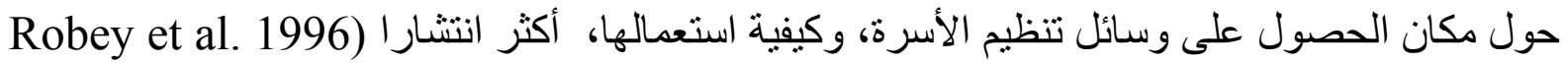

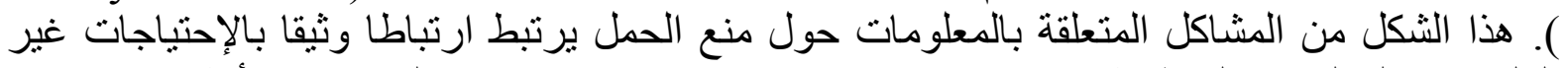

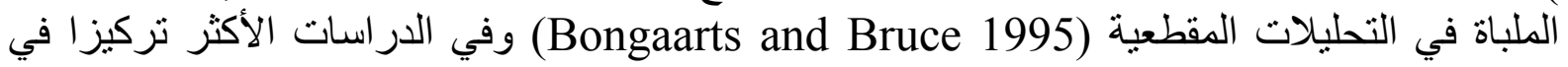
بواكستان (Population Council/Islamabad 1997)، وشمالي الهند ( Viswanathan et al. .(1998; Mishra et al. 1999

إن السببين الثاني والثالث - أي المعارضة الاجتماعية والمخاوف الصحية ـ هما أبرز ما يساق فيما

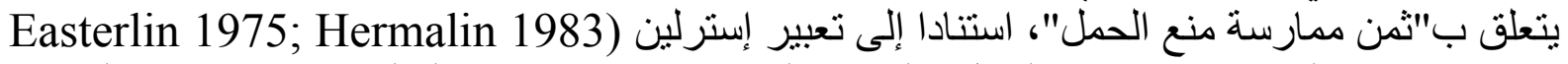

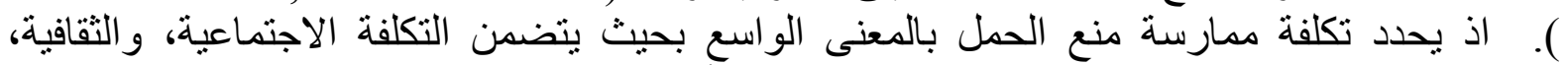

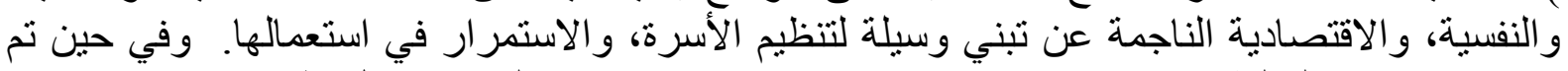

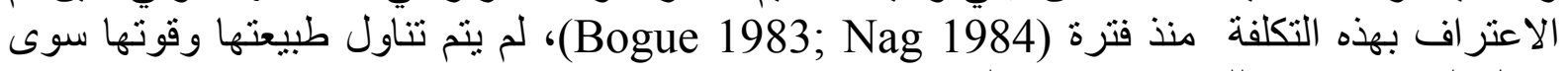
خلال التسعينيات، وذلك في عديد من المو اقع.

لقد ركزت البحوث المتعمقة الحديثة على الاحتباجات غير الملباة في صفوف النساء؛ ومن غير الغريب

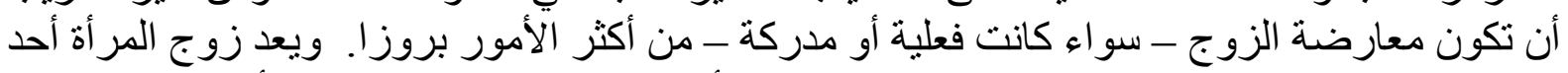

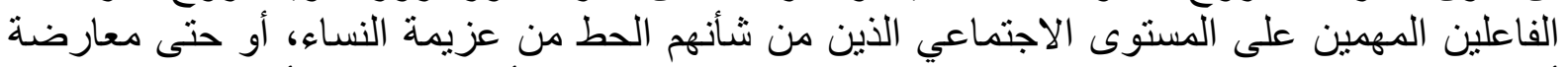

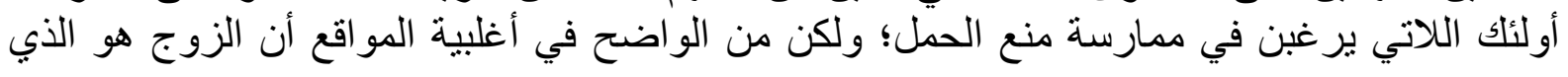


يمثل أكثر المؤثرات سيطرة. فقد تم تحديد معارضة الزوج كسبب أساسي لعدم ممارسة منع الحمل في

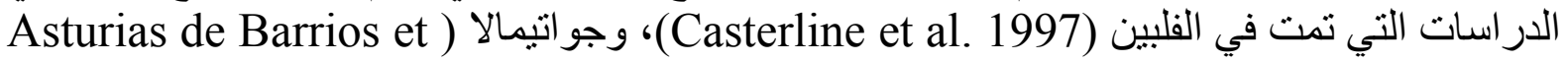

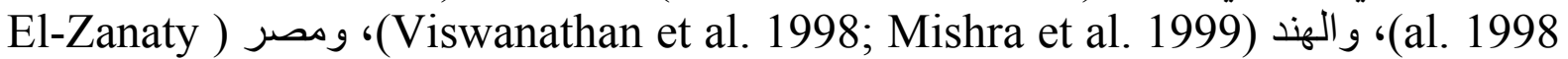

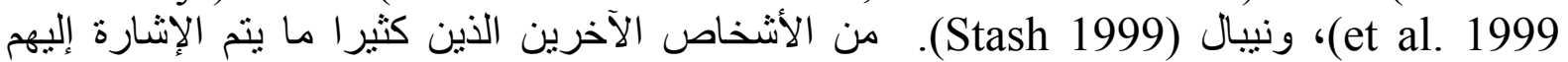

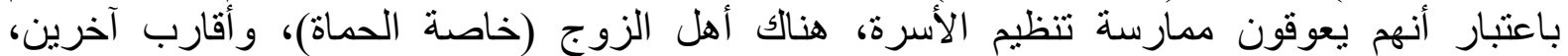
و الجيران، و القيادات السياسية أو الدينية في المجتمع المحلي.

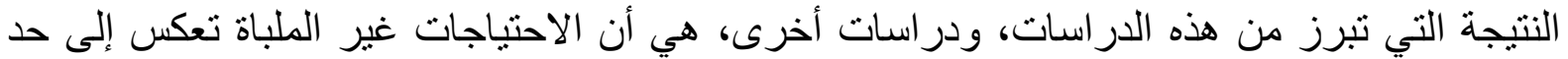

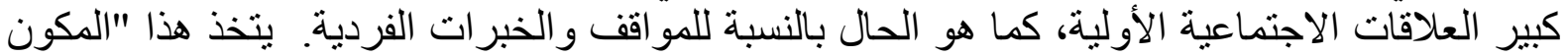

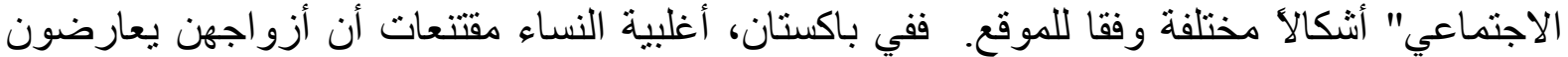

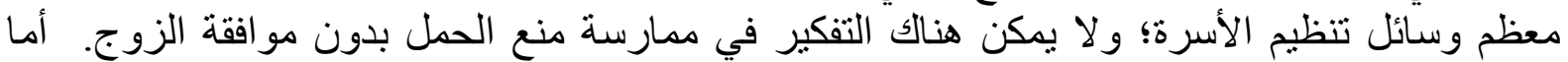

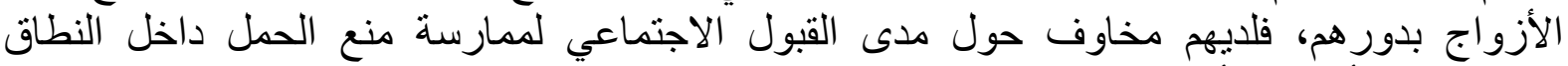

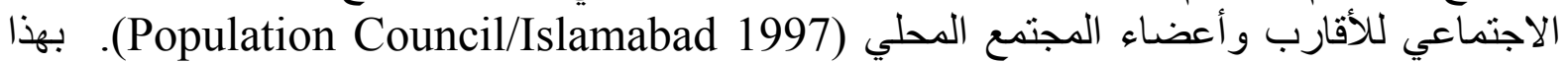

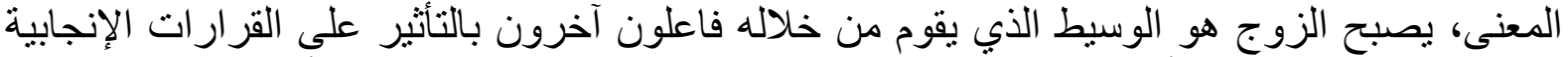

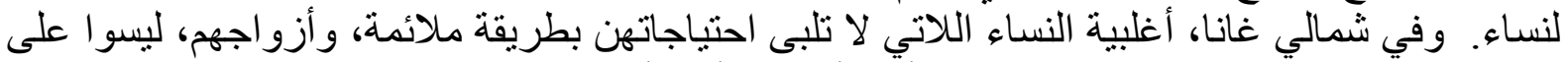

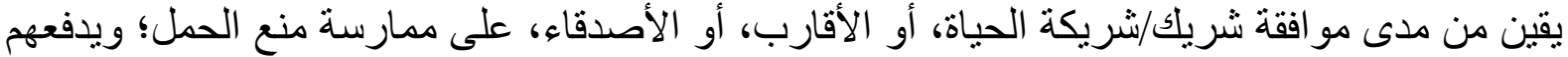

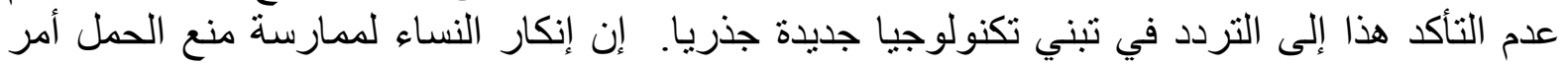

شائع (Biddlecom et al. 1998).

لقد كثفت دراسات عملية حديثة العمليات التي تخلق من خلالها العلاقات الزورجية معوقات أمام ممارسة

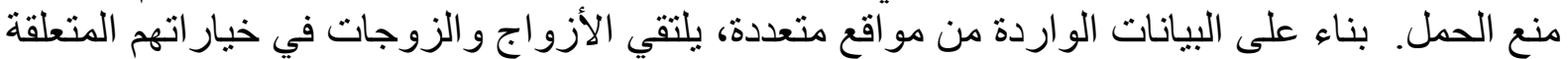

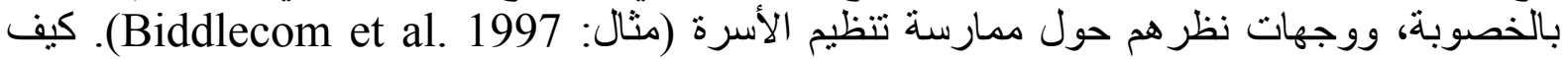

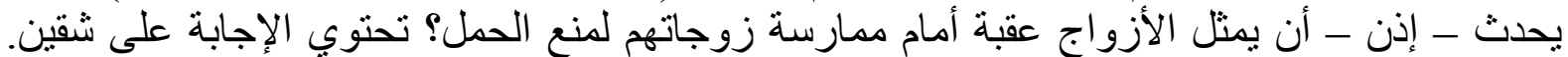

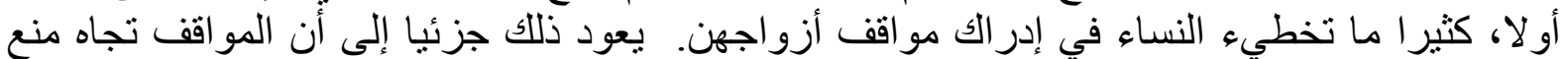

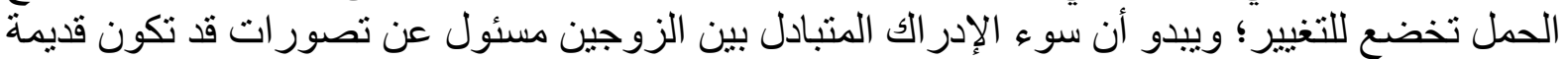

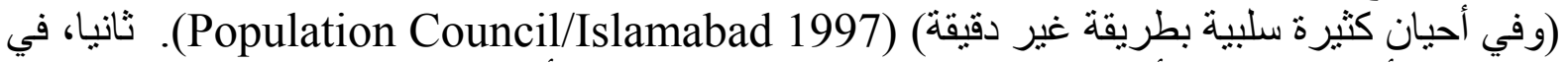

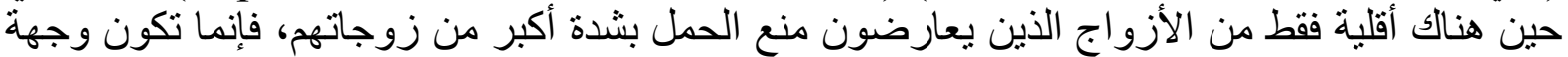

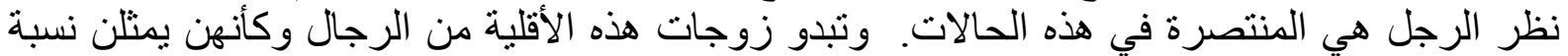
غير متكافئة من النساء اللاتي لا تلبى احتياجاتهن بطريقة ملائمة (Biddlecom et al. 1997).

هناك نتيجة أخرى واسعة الانتشار، وهي أن المخاوف الصحية تمثل عقبة أساسية أمام تبني ممارسة

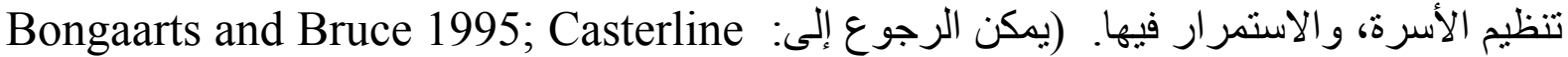
et al. 1997; Asturias de Barrios et al. 1998; Viswanathan et al. 1998; Yinger الإنبام 1999 الأسرة بالإشارة على مدى عقود إلى المخاوف الصحية. توفر الداراسات العملية الحديثة تأكيداً أكثر دقة

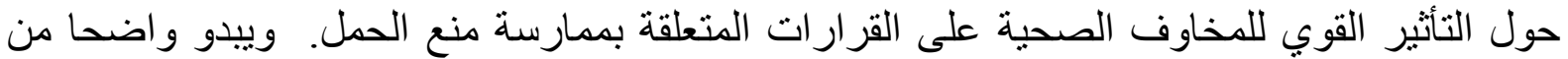

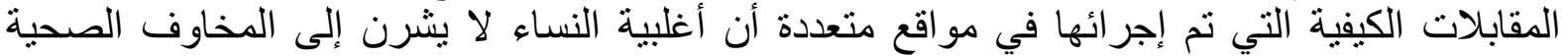

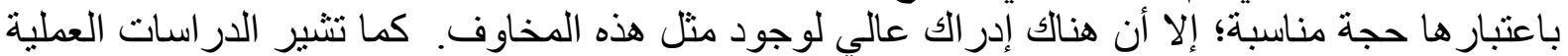

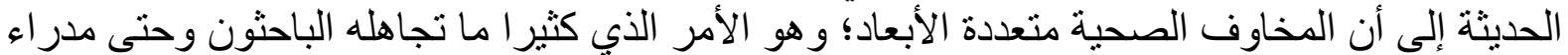

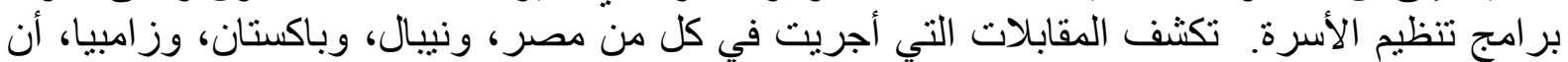

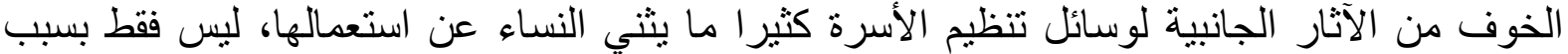

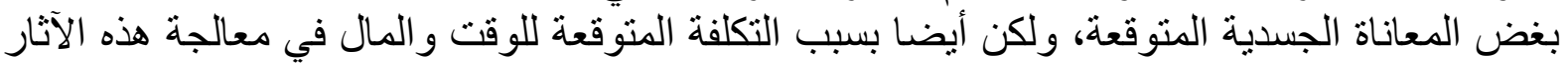

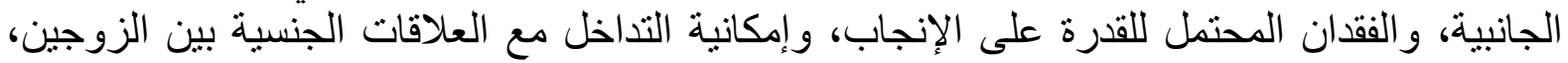


و الثعور بأن الآثار الجانبية تعني عدم الموافقة الإلهية. و وعند التعرف على الطبيعة متعددة الأبعاد لهذه التكلفة، يتضح أن المخاوف الصحية هي السبب وتئية الصنل عقبة مهمة أمام ممارسة تنظيم الأسرة.

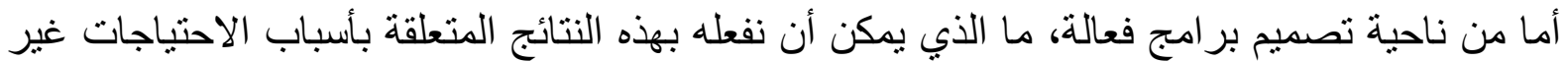

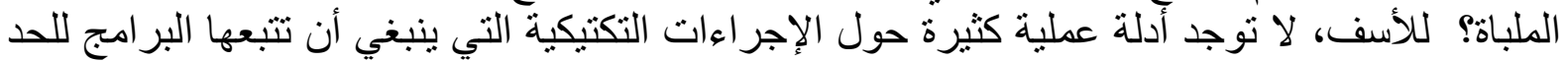

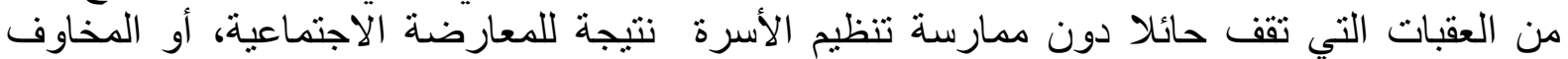

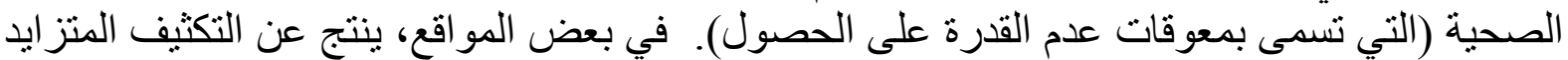

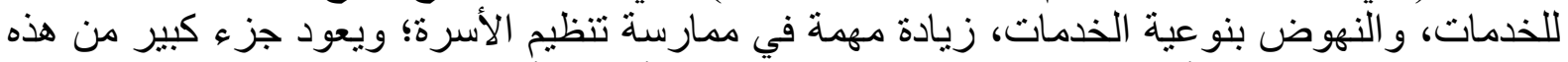

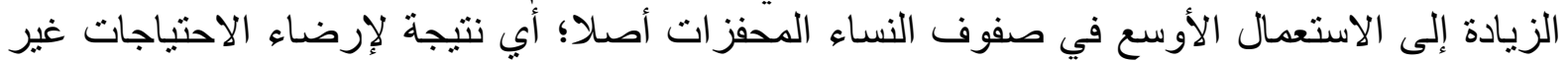

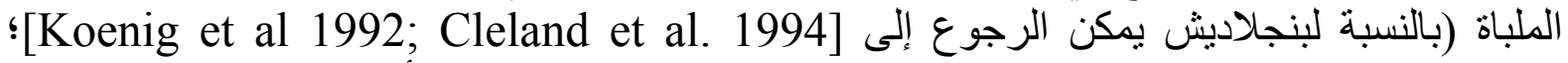

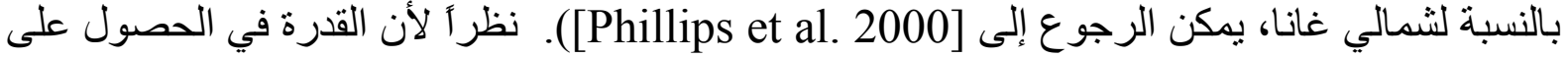

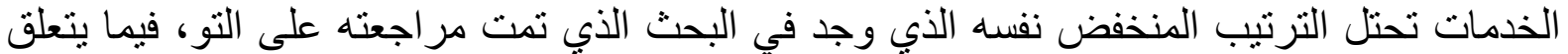

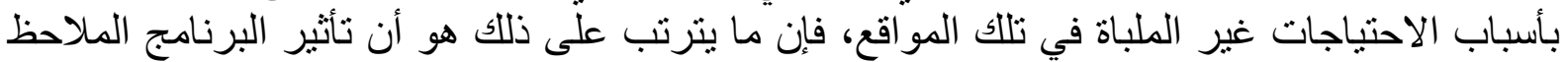

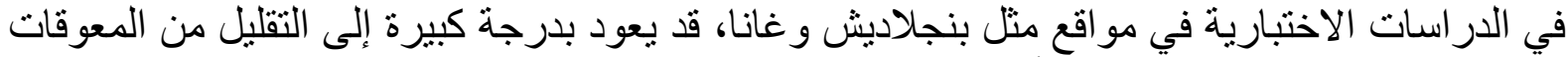

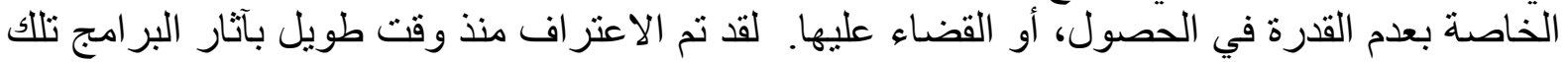

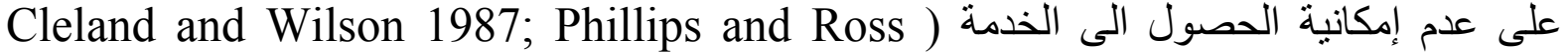

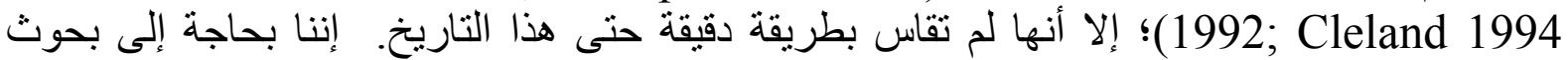

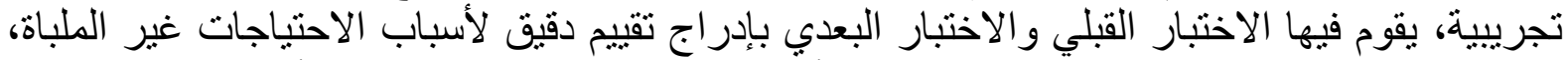

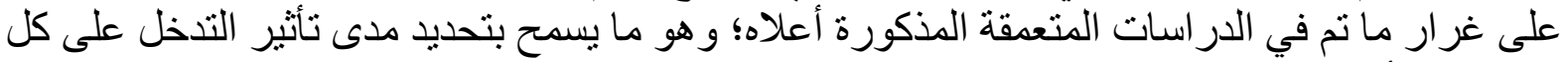

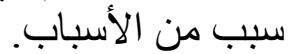

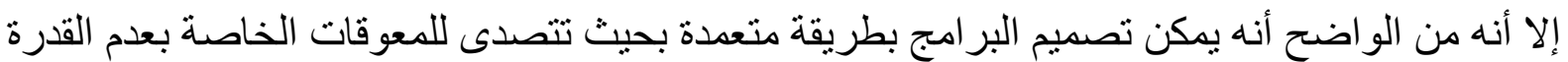

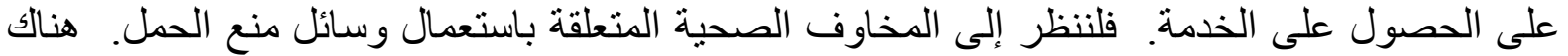

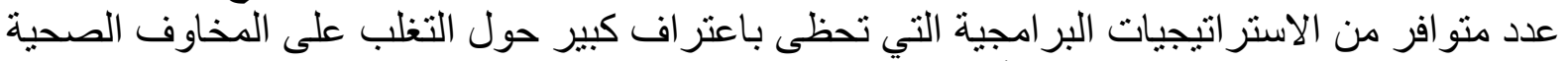

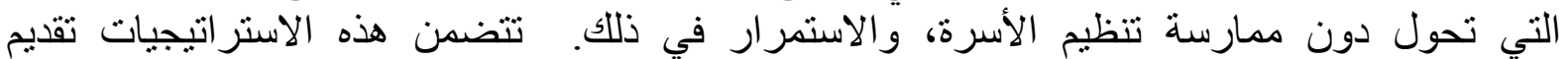

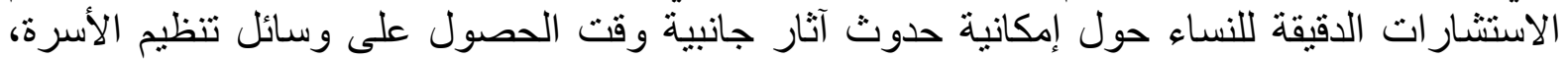

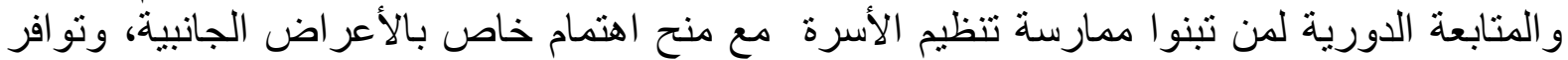

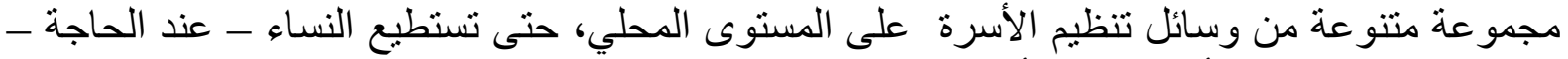

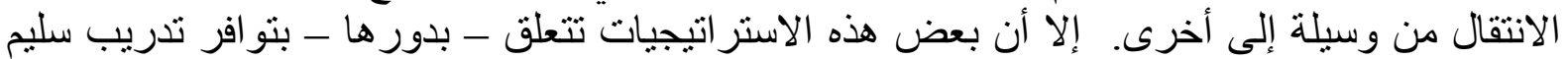

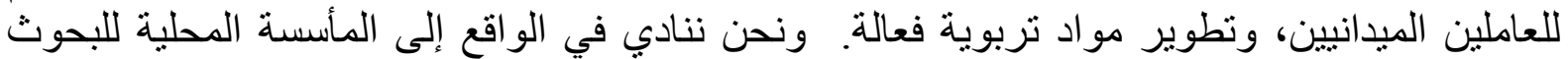

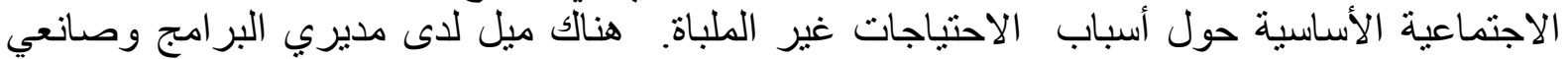

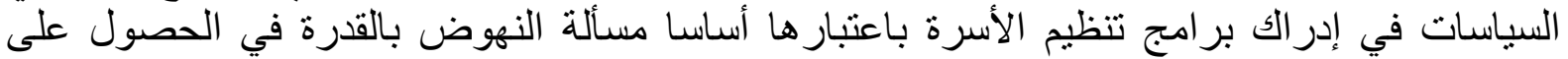

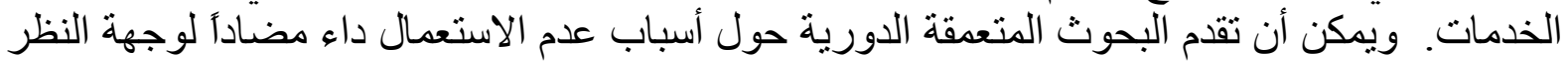
الضيقة تللك حول ما يمكن أن تحققه البر امج البحت

\section{الاحتياجات غير الملباة والسياسة السكانية}

لقد مثل التساوي بين السياسة السكانية و الهدف الأوحد للنهوض بخدمات تتظيم الأسرة (على سبيل المثال

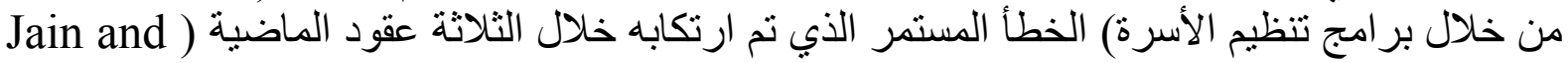

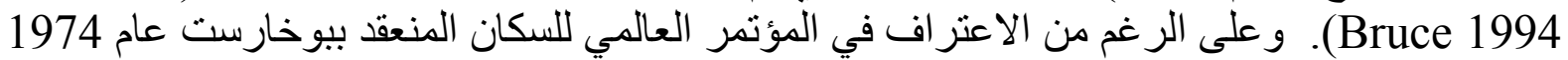

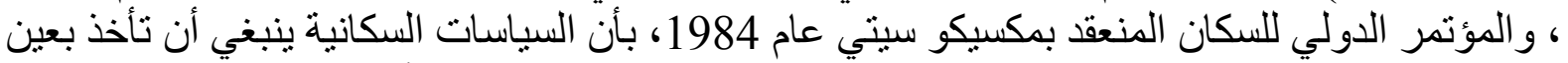

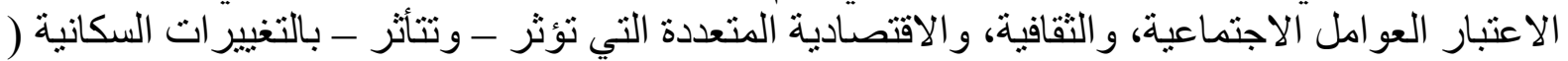
1984 
بتتظيم الأسرة. وفي حين بلورت أغلبية البلان سياسات تعبر أهمية إلى زيادة التحاق الإناث بالمدرسة،

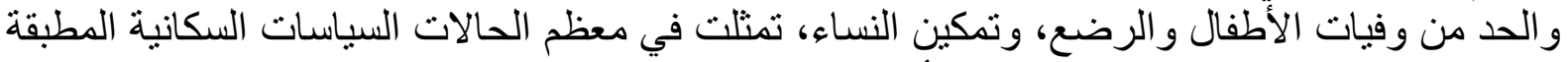

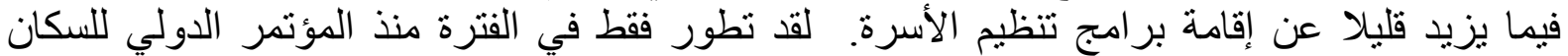

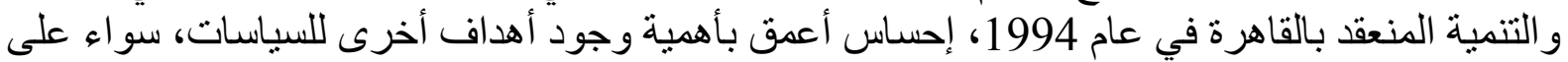

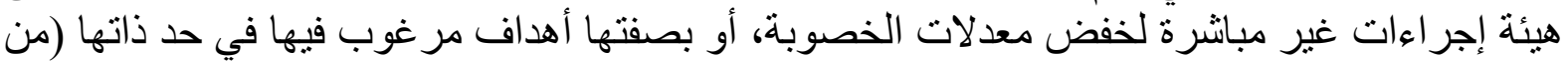

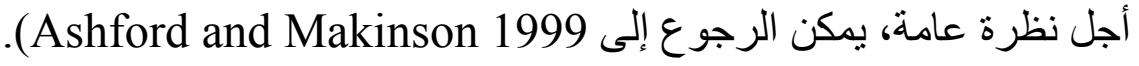

يمكن الجدل بأن التركيز على الوقاية من الحمل غير المخطط له يبقي على اهتمام السياسات السكانية

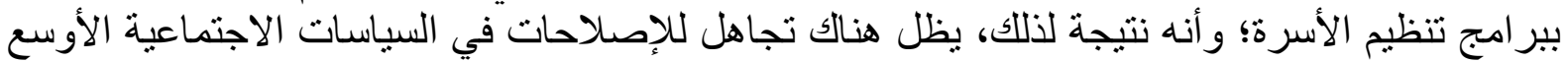

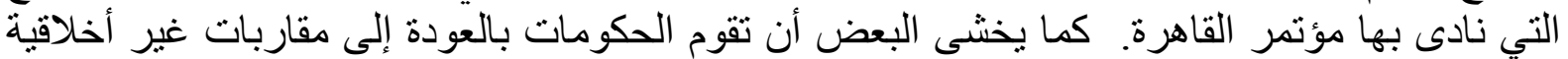

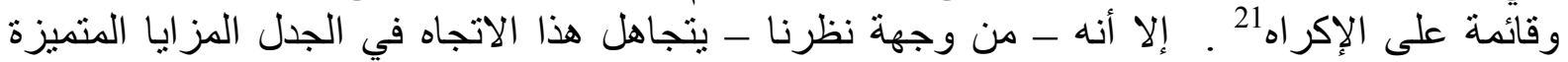

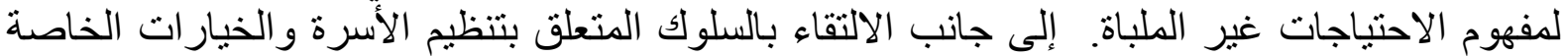

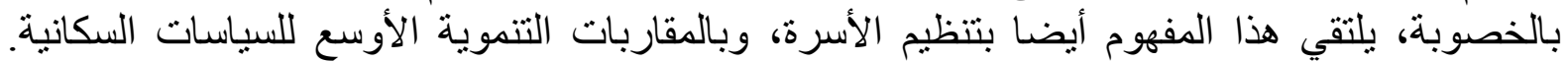

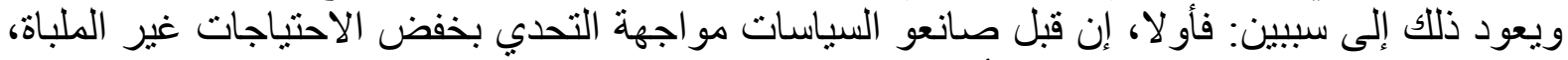

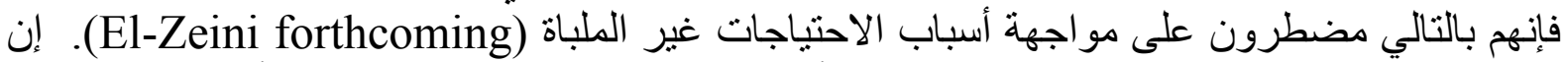

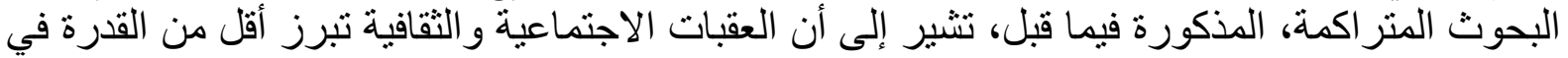

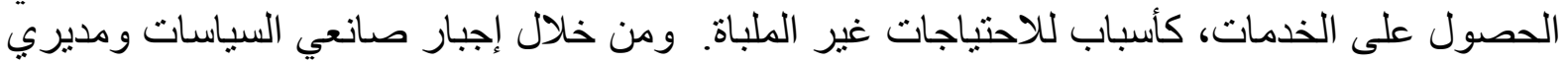

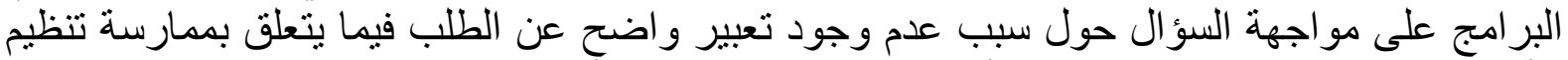

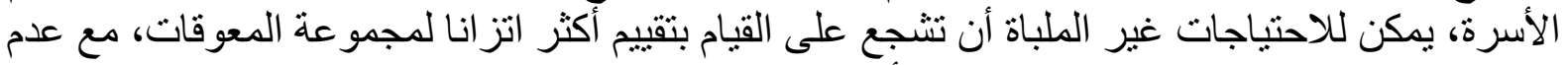

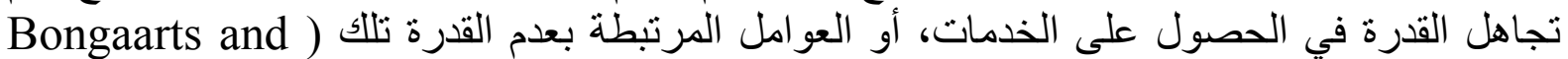

.(Bruce 1995

أما الوسيلة الثانية - و الأكثر عمومية ـ التي تقوم بواسطتها الاحتياجات غير الملباة بربط تنظيم الأسرة

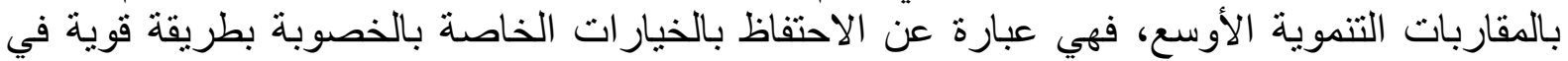

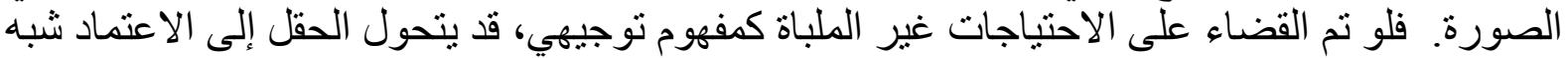

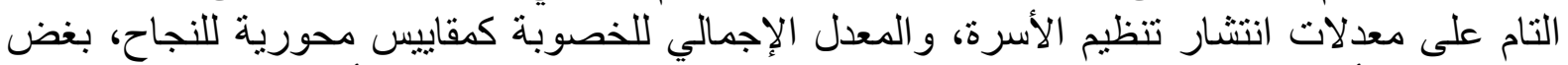

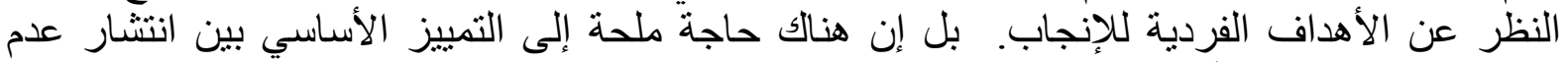

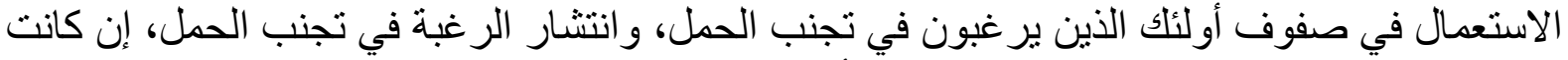

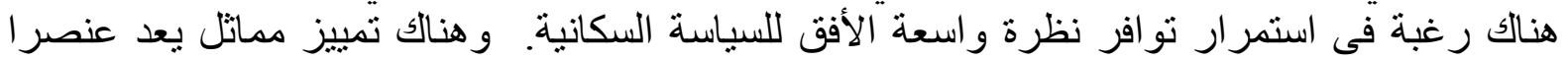

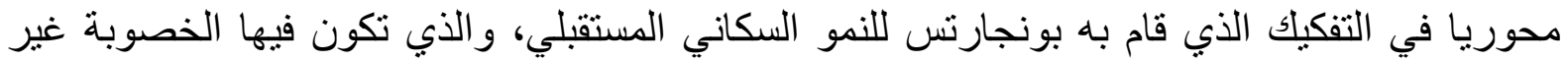

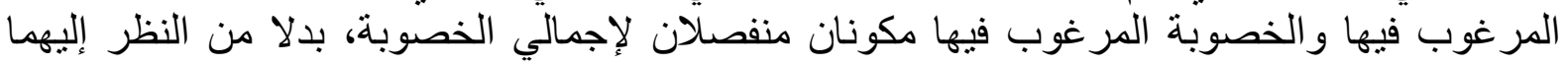

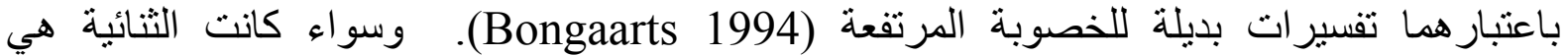

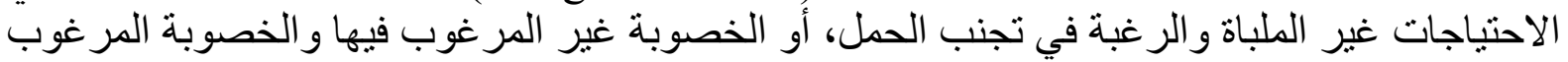

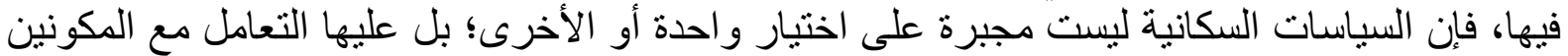

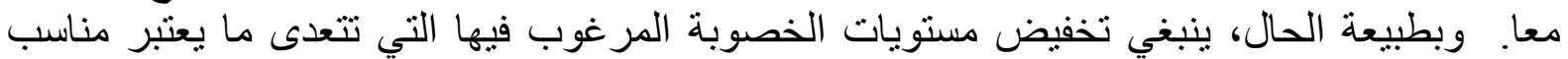

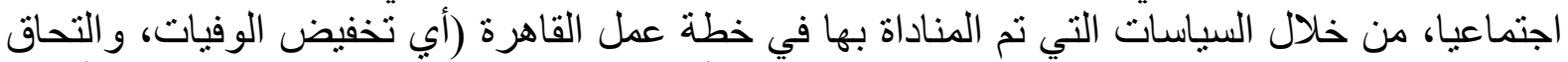

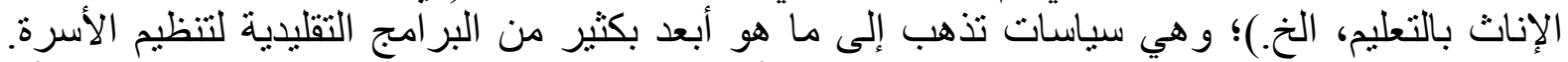

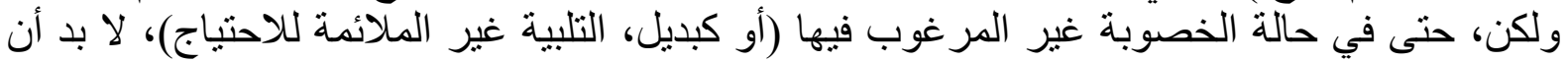

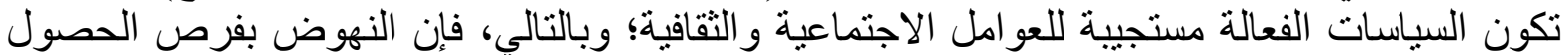
على خدمات تنظيم الأسرة لا يكفي في حد ذاته.

هناك مبرر آخر للاحتفاظ بالاحتياجات غير الملباة كحجر زاوية للسياسات السكانبة؛ فمن خلال اعتبار

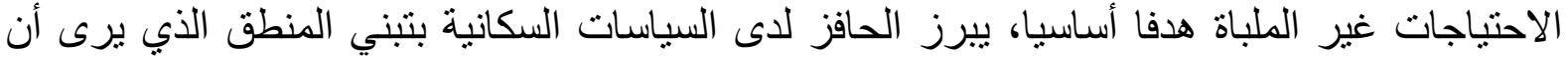


مساعدة الأفر اد على تحقيق تطلعاتهم الشخصية يعد هدفا مشرو عا للسياسات العامة. و ونحن نزعم أن هذا

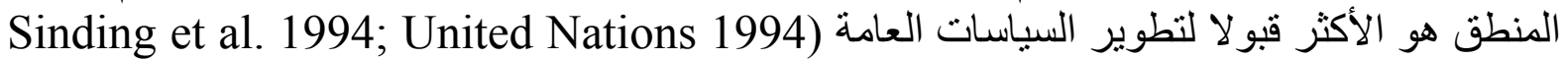

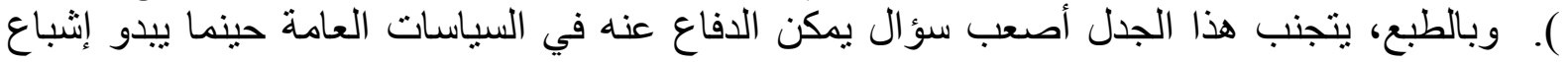
التطلعات الفردية متناقضا مع الصالح العام (Demeny 1986; Lee 1990; MacKellar 1997).

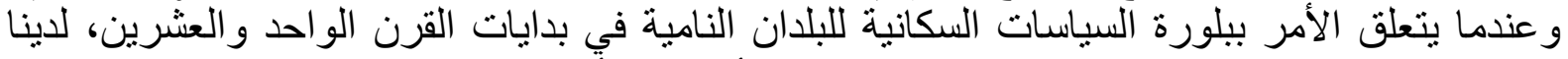

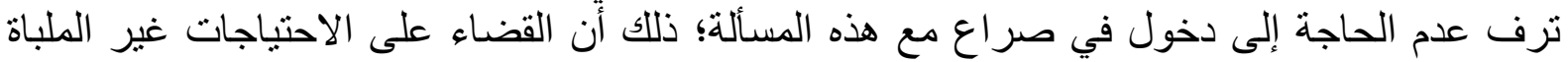
سوف يؤدي في جميع البلدان النامية - نظرياً - إلى انتقال السلوك الإنجابي في اتجاه إيجابي بالنسبة للصالح العام.

ويمكن القول بعبارة أكثر عملية الاحتياجات غير الملباة تولد الخصوبة غير المرغوب فيها؛ وتظل هذه

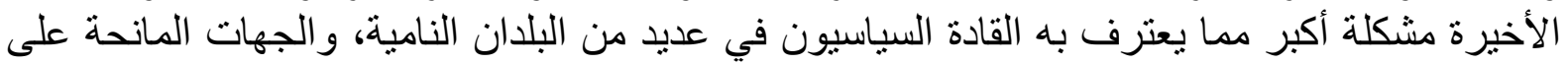

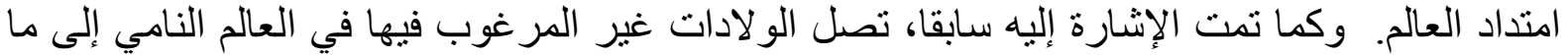

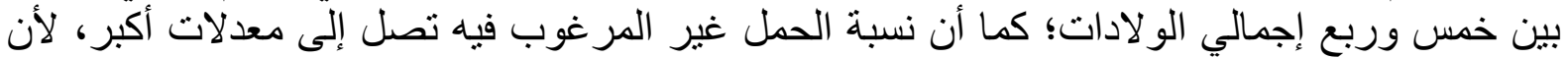
بعض هذا الحمل يتم التخلص منه عن طريق الإجهاض. هناك انطباع مستمر في عديد من العو اصم بأن التان

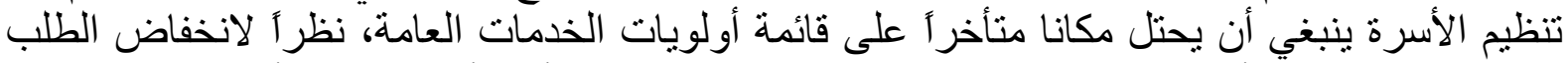

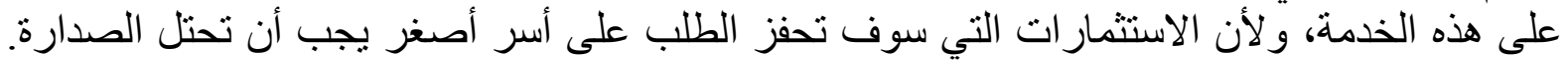

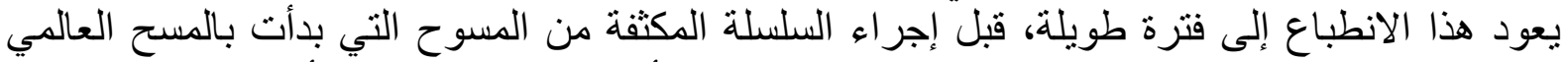

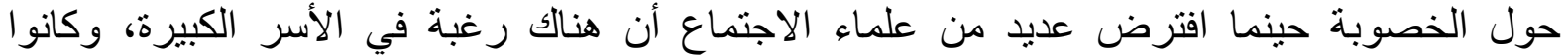

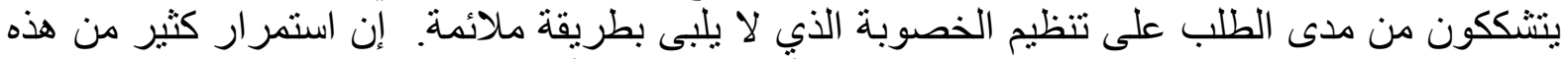

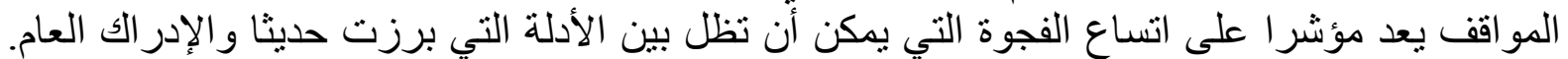

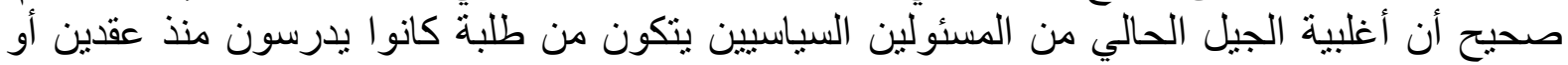
ثناثة، و هم يحتفظون بوجهة نظظر عتيقة حول الخصوبة في البلدان النامية.

وفى سياق السؤال حول الحاجة إلى الاهتمام بالاحتياجات غير الملباة إلى تنظيم الأسرة في تصميم

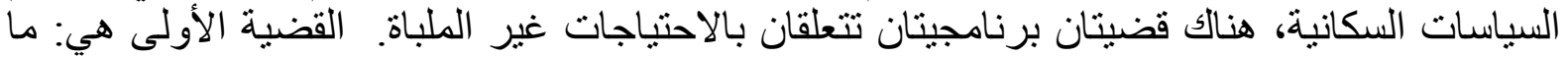

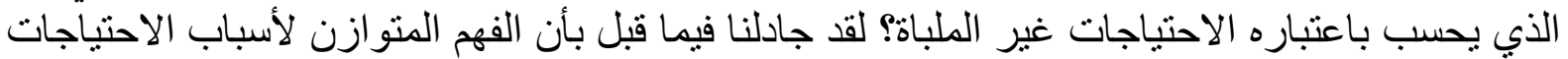

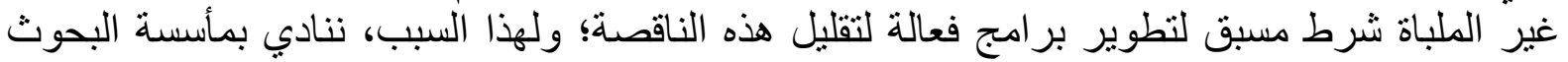

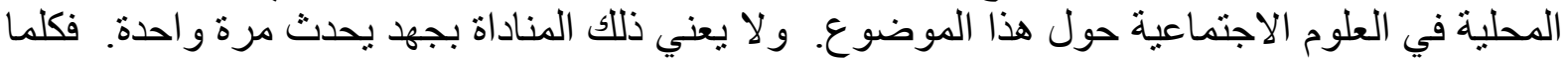

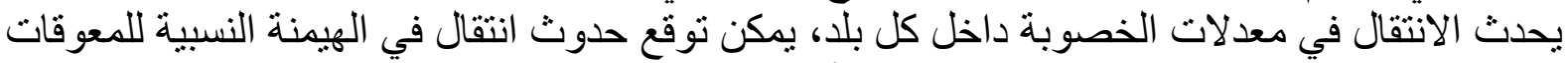

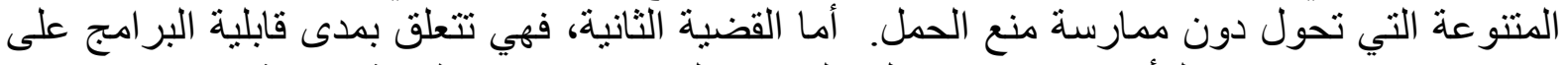

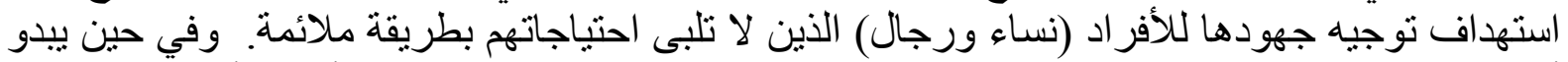

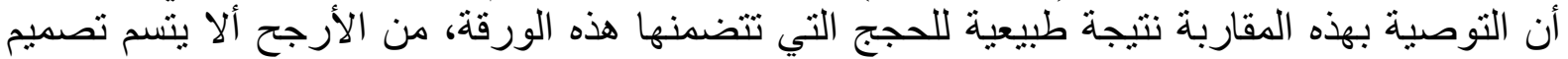

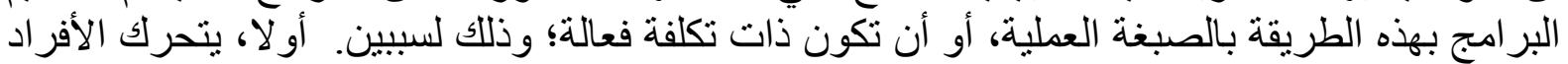

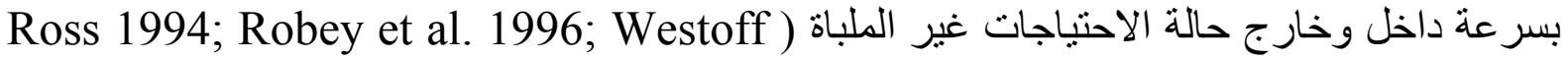
1999 التي لا تلبى احتياجاتها بطريقة ملائمة هدف متحرك (El-Zeini forthcoming). و وثانيا، لأن متابعة

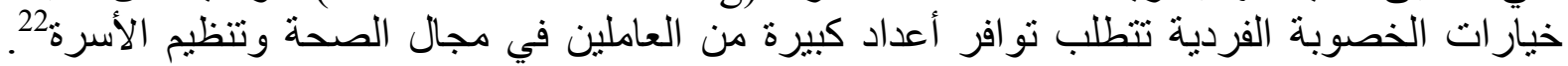

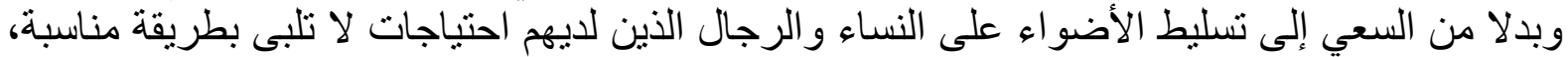

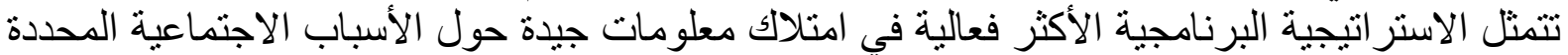

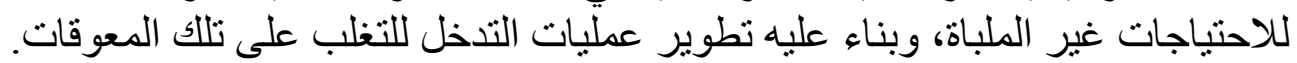


1م: إجراء أغلبية هذه الدرلسلت المتعقة في إطار مشروعلن يضملن بلدان متعدة حول الاحتيلجلت غير الملبة، هما: المشروع الذي أجره

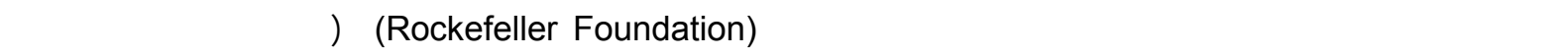

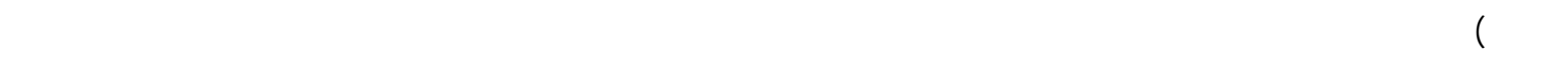

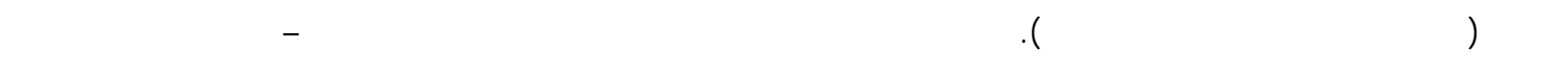

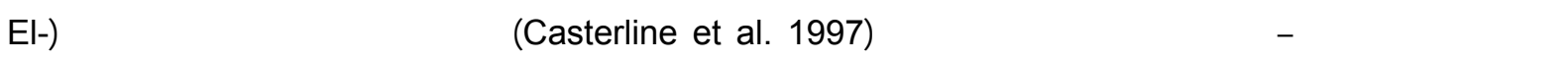

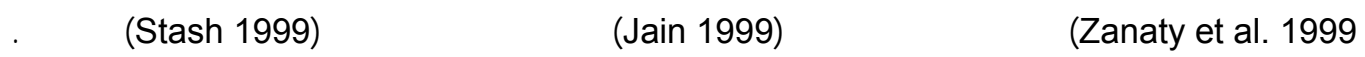

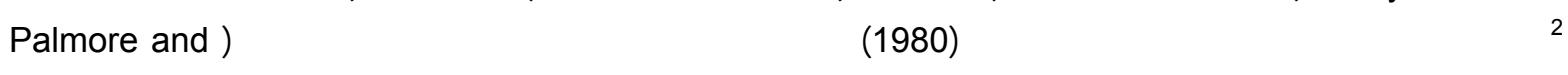
(Concepcion العالمية حول الخصوبة تناوك هذه العلاقة (مثل: Ochoa 1982; Pullum et a. 1984: Johnson-Acsadi and Szykman

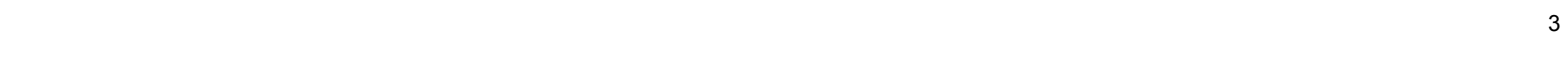

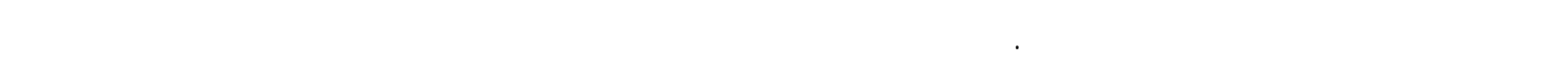
تضمينا لنسبة النساء اللاتي يتمنين تجنب الحطل ولكنهن لا يستعملن وسالل منع الحطل.

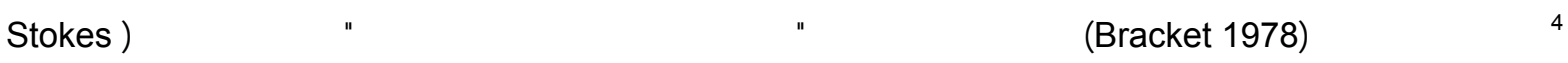

مَّن الصعب التصور أن ظهور فكرة تحقيق الظلعلت الإنجابية للأفرادسوف تلبي الأهدف الاجتماعية بطريقة كبيرة، قد جاء مع مؤتمر

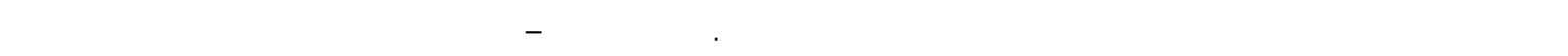

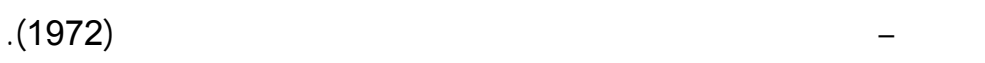

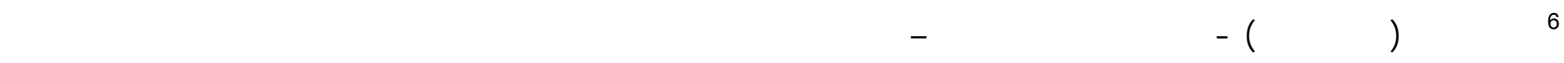

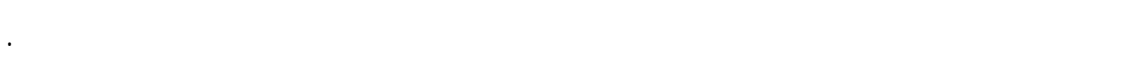

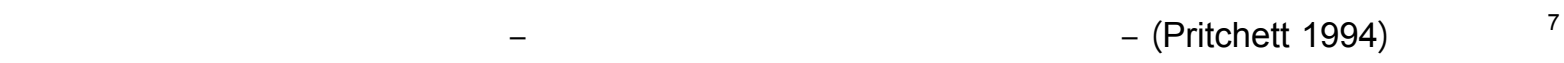

المتعلة بالخصوبة.

أغير أن في الدرلسكت التي لجريت في أفريقيا تهت الصحراء ) Mushingeh and Kurz 1998; Biddlecom et al. 1998;

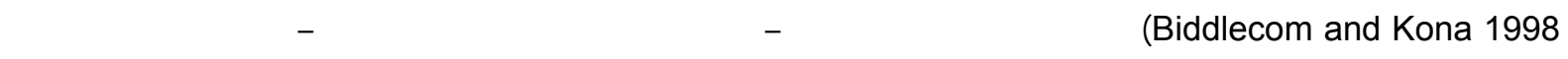

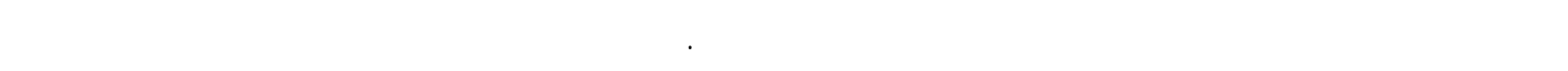

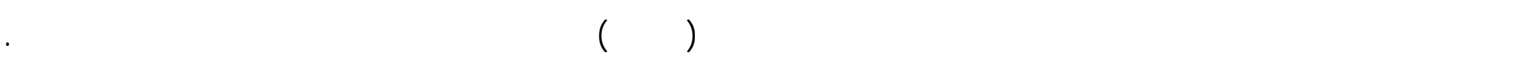

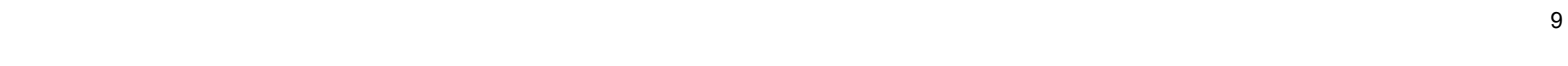

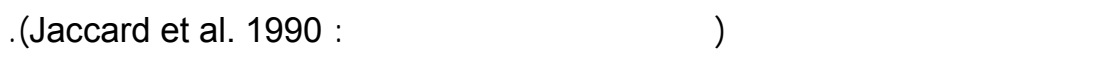
إبئي

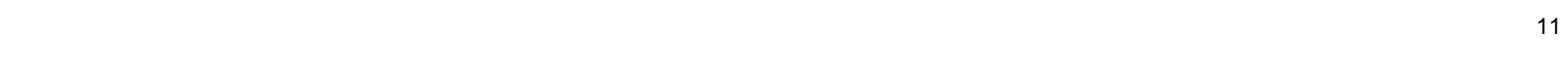

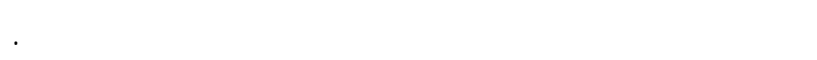

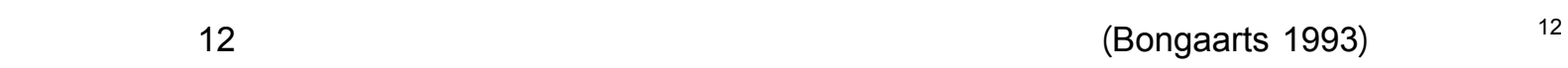

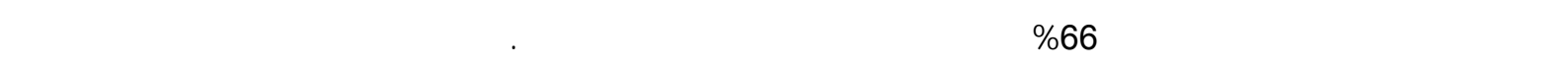

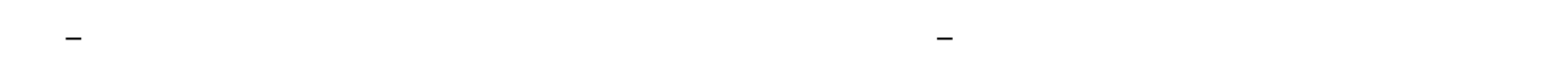

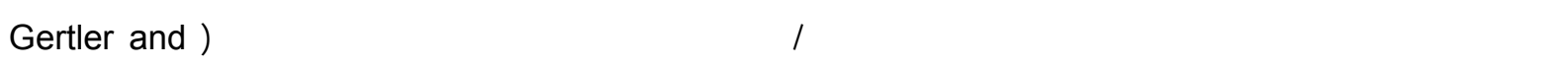

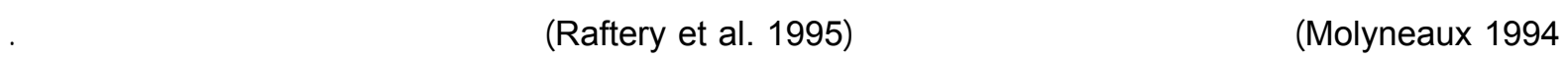

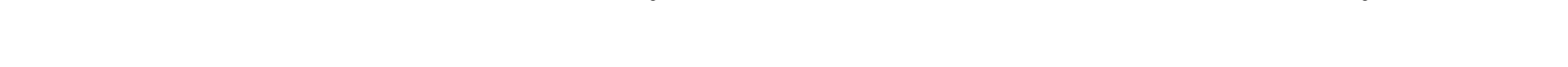

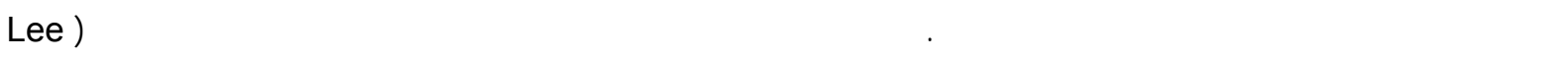

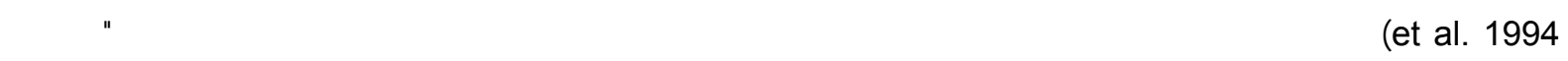

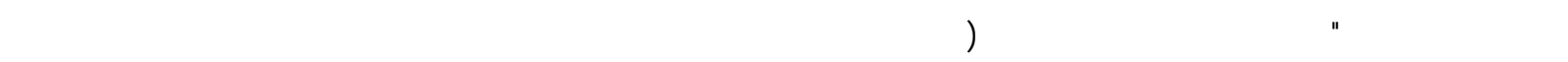




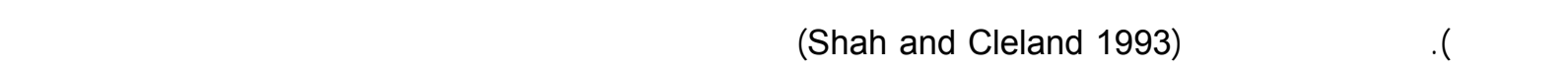

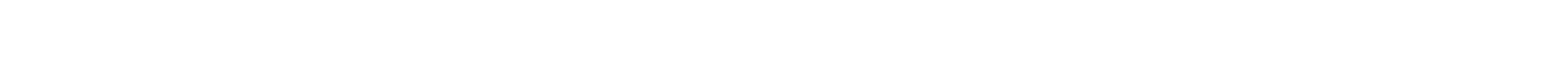

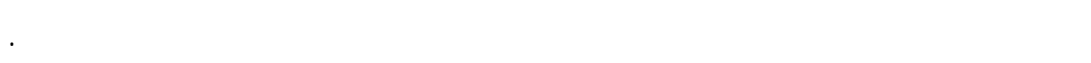

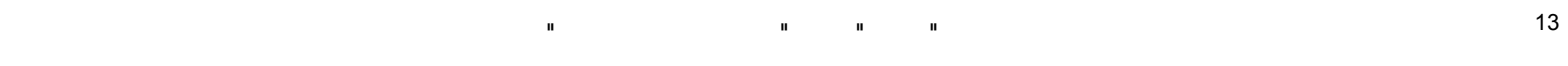

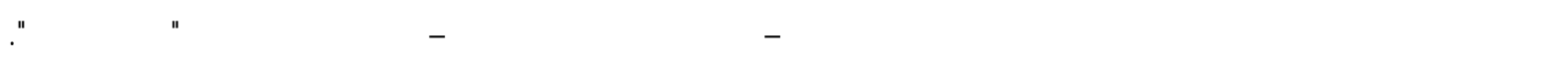

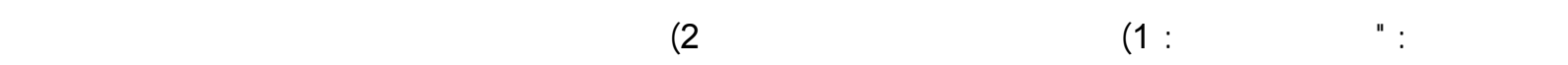

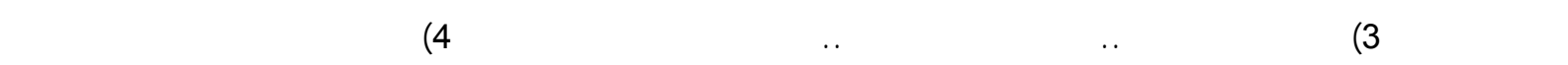

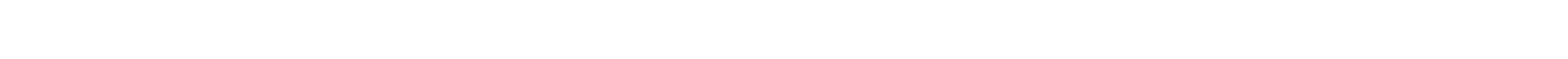

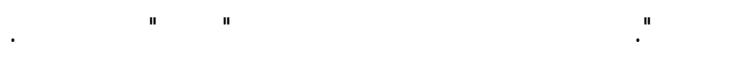

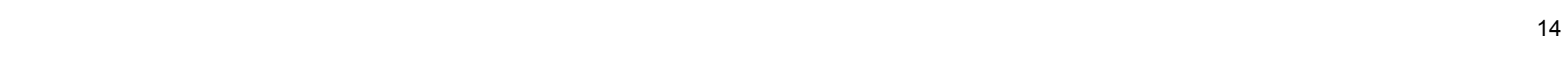

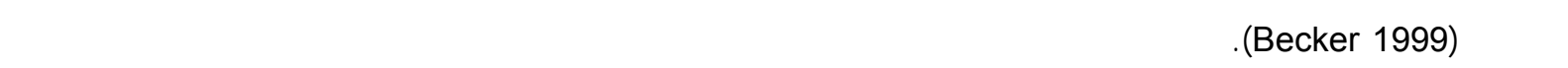

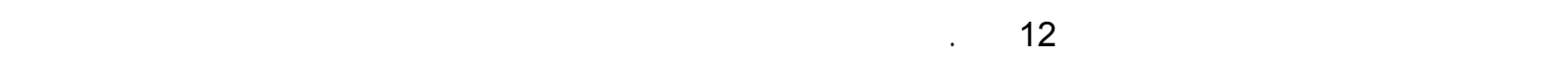

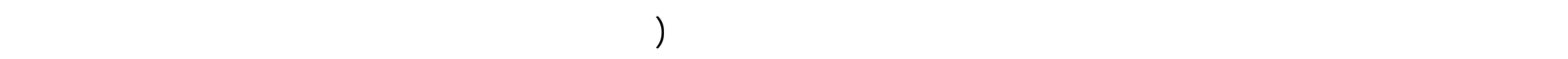

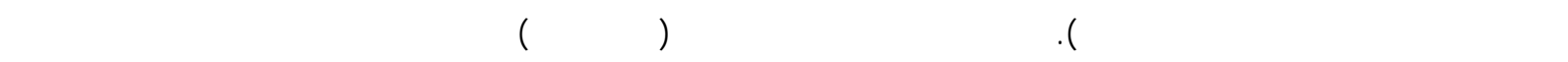

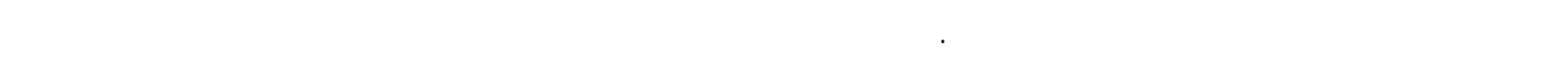

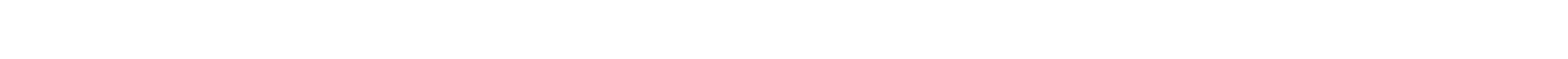

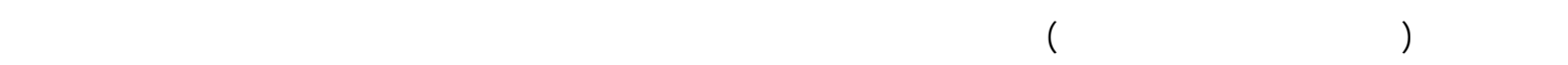

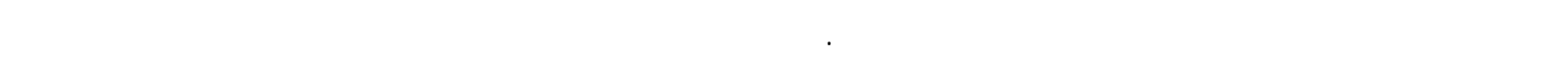

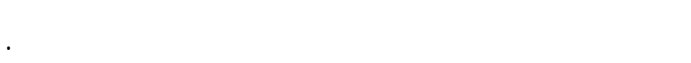

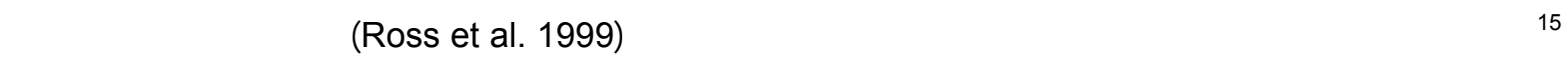

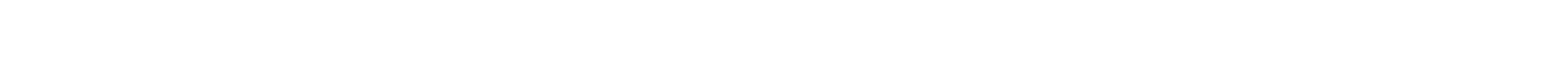

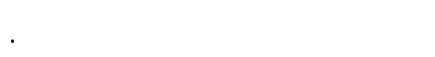

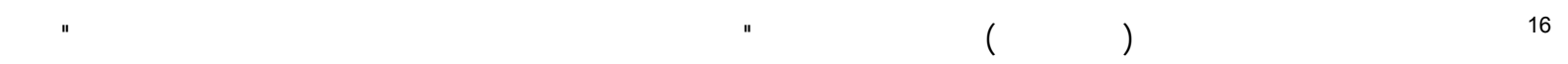

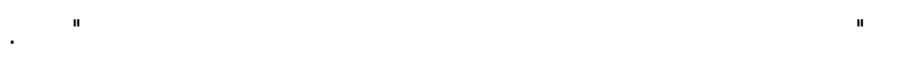

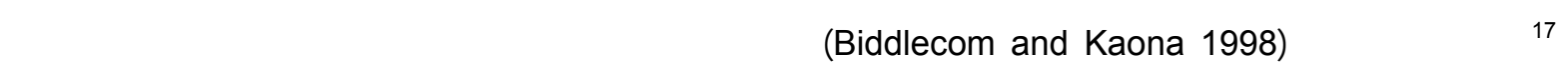
جواتيمالا وزلمبيا (Asturias de Barrios et al. 1998; Mushingeh and Kurz 1998)، لطنتثاء لهذا.

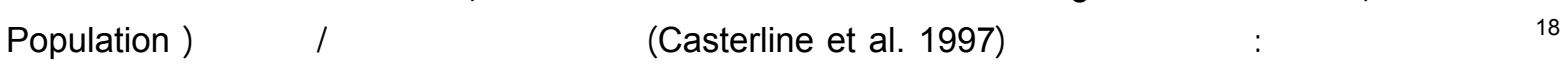

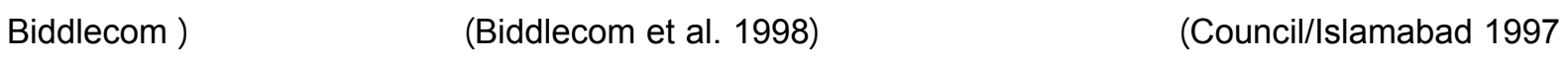

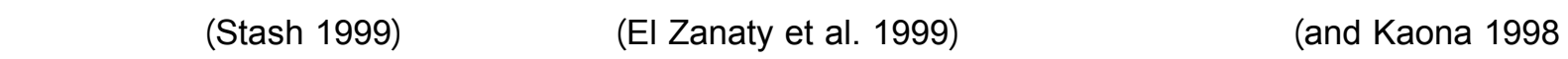

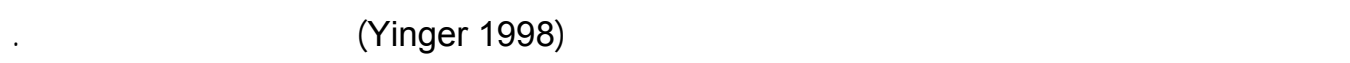

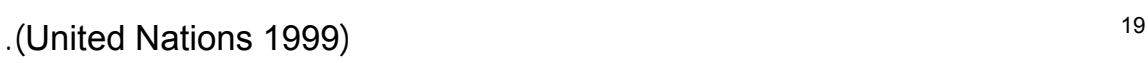

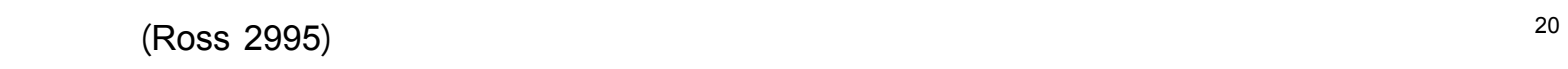

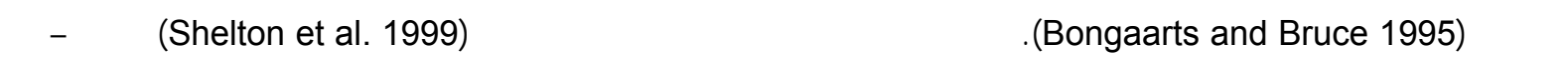

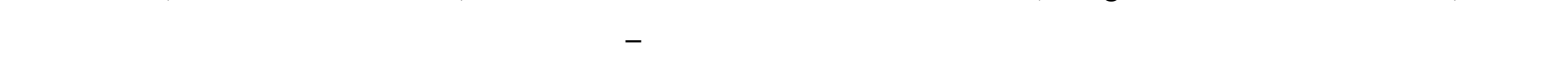

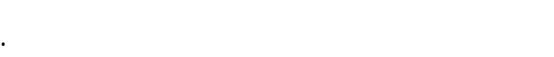

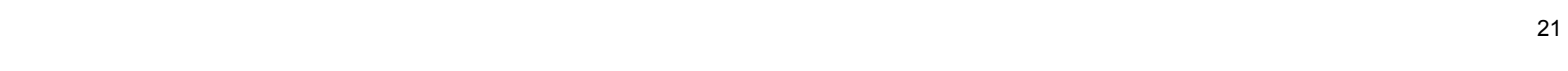

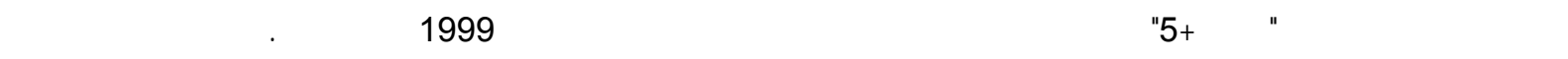

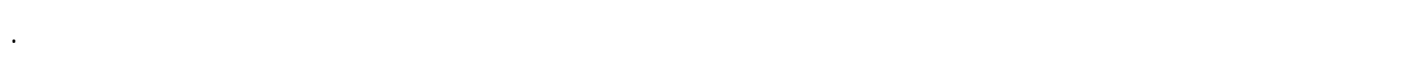

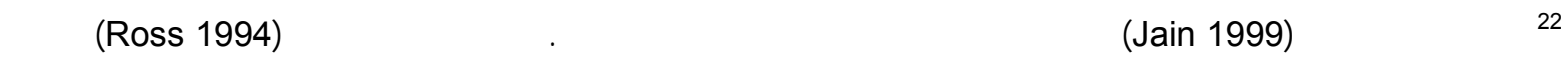
وآخرون (Robey et al. 1996) أنه بينما يتحرك الأفراد دلظل وخان حان حالة التلبية غير الملائمة للاحتياج، قد قطل الخصائص 
الديموجرافية والاجتماعية، والاقتصادية لهؤلاء الأشخاص ثابتة. وقيم ذلك بعض الأسبلس للمستهدف، بعدما تكون البحوث قد حددت الخصائص المهيمنة للمأشخاص النين لا تلب لحتيلجاتهر بطريقة ملائمة في موقع محدد.

\section{References}

Adler, Nancy E., Susan M. Kegeles, Charles E. Irwin, and Charles Wibbelsman. 1990. "Adolescent contraceptive behavior: An assessment of decision processes," Journal of Pediatrics 116(3): 463-471.

Adetunji, Jacob A. 1998. Unintended Childbearing in Developing Countries: Levels, Trends, and Determinants. Demographic and Health Surveys Analytical Reports No. 8. Calverton, MD: Macro International Inc.

Ajzen, Icek. 1993. "Attitude theory and the attitude-behavior relation," in D. Krebs and P. Schmidt (eds.), New Directions in Attitude Measurement. Berlin: Walter de Gruyter, pp. 41-57.

Alan Guttmacher Institute. 1999. Sharing Responsibility: Women, Society and Abortion Worldwide. New York: Alan Guttmacher Institute.

Anderson, John E. and Leo Morris. 1981. "Fertility differences and the need for family planning services in five Latin American countries," International Family Planning Perspectives 7(1): $16-21$.

Ashford, Lori and Carolyn Makinson. 1999. Reproductive Health in Policy and Pra tice. Washington, DC: Population Reference Bureau.

Asturias de Barrios, Linda et al. 1998. Unmet Need for Family Planning in a Peri- Urban Community of Guatemala City. Washington, DC: International Center for Research on Women.

Bankole, Akinrinola and Alex Chika Ezeh. 1997. "Unmet need for couples: A conceptual framework and evaluation with recent DHS data," paper presented at the Annual Meeting of the Population Association of America, Washington, DC, March.

Bankole, Akinrinola and Charles F. Westoff. 1998. "The consistency and validity of reproductive attitudes: Evidence from Morocco," Journal of Biosocial Science 30(4): 439-455.

Becker, Stan. 1999. "Measuring unmet need: Wives, husbands or couples?" Interna tional Family Planning Perspectives 25(4): 172-180.

Berelson, Bernard. 1969. "Family-planning programs and population control," in Bernard Berelson (ed.), Family-Planning Programs: An International Survey. New York: Basic Books, pp. 291303.

Bhatia, Shushum. 1982. "Contraceptive intentions and subsequent behavior in rural Bangladesh," Studies in Family Planning 13(1): 24-31.

Biddlecom, Ann E., John B. Casterline, and Aurora E. Perez. 1997. "Spouses' views of contraception in the Philippines," International Family Planning Perspectives 23(3): 108-115.

Biddlecom, Ann E. and Fred Kaona. 1998. "The nature of unmet need for contraception in an urban African setting," paper presented at IUSSP Seminar on Fertility Transition in sub- 
Saharan Africa, Nairobi, November.

Biddlecom, Ann E., Eva Tagoe-Darko, and Kubaje Adazu. 1998. "Unmet need for con- traception in a traditional West African setting," manuscript, Policy Research Division, Population Council, New York.

Bogue, Donald J. 1974. "Population perspectives: Some views from a sociologist," Population Dynamics Quarterly 2(2): 2-20.

. 1983. "Normative and psychic costs of contraception," in R. A. Bulatao and

R. D. Lee (eds.), The Determinants of Fertility in Developing Countries, Volume

2. New York: Academic Press, pp. 151-192.

Bongaarts, John. 1993. "The supply-demand framework for the determinants of fertility: An alternative implementation," Population Studies 47(3): 437-456.

. 1994. "Population policy options in the developing world," Science 263: 771-776.

. 1997a. "The role of family planning programmes in contemporary fertility transitions," in G. W. Jones, R. M. Douglas, J. C. Caldwell, and R. M. D’Souza

(eds.), The Continuing Demographic Transition. New York: Oxford University

Press, pp. 422-443.

1997b. "Trends in unwanted childbearing in the developing world," Studies in Family Planning 28(4): 267-277.

Bongaarts, John and Judith Bruce. 1995. "The causes of unmet need for contraception and the social content of services," Studies in Family Planning 26(2): 57-75.

Brackett, James W. 1978. "Family planning in four Latin American countries: Knowledge, use, and unmet need," International Family Planning Perspectives and Digest 4(4): 116-123.

Bruce, Judith. 1990. "Fundamental elements of the quality of care: A simple framework," Studies in Family Planning 21(2): 61-91.

Casterline, John B., Aurora E. Perez, and Ann E. Biddlecom. 1997. "Factors underlying unmet need for family planning in the Philippines," Studies in Family Planning

28(3): 173-191.

Casterline, John B., Zeba Sathar, and Minhaq ul Haque. 2000 (forthcoming). "Obstacles to contraceptive use in Pakistan: A study in Punjab," Policy Research Division Working Paper. New York: Population Council.

Cleland, John. 1994. "Different pathways to demographic transition," in F. Graham- Smith (ed.), Population-The Complex Reality. Golden, CO: North American Press, pp. 229-247.

Cleland, John, James F. Phillips, Sajeda Amin, and G. M. Kamal. 1994. The Determinants of Reproductive Change in Bangladesh: Success in a Challenging Environment. Washington, DC: The World Bank.

Cleland, John and Christopher Wilson. 1987. "Demand theories of the fertility transition: An iconoclastic view," Population Studies 41(1): 5-30.

Curtis, Siân L. and Charles F. Westoff. 1996. "Intention to use contraceptives and subsequent contraceptive behavior in Morocco," Studies in Family Planning 27(5): 239-250. 
Davis, Kingsley. 1967. "Population policy: Will current programs succeed?" Science 158: 730-739.

Dawes, Robyn M. 1998. "Behavioral decision making and judgment," in D. T. Gilbert, S. T. Fiske, and G. Lindzey (eds.), The Handbook of Social Psychology, Volume I. Boston: McGraw-Hill, pp. 497-548.

DeGraff, Deborah S. and V. de Silva. 1996. "A new perspective on the definition and measurement of unmet need for contraception," International Family Planning Perspectives 22(4): 140-147.

Demeny, Paul. 1975. Letter to the editor, Scientific American, 232(5), May. 1986. "Population and the invisible hand," Demography 23(4): 473-487.

De Silva, W. Indralal. 1991. "Consistency between reproductive preferences and behavior: The Sri Lankan experience," Studies in Family Planning 22(3): 188-197.

Dixon-Mueller, Ruth. 1993. Population Policy and Women's Rights: Transforming Reproductive Choice. New York: Praeger.

Dixon-Mueller, Ruth and Adrienne Germain. 1992. "Stalking the elusive 'unmet need' for family planning," Studies in Family Planning 23(5): 330-335.

Dodoo, F. Nil-Amoo, Y. Luo, and E. Panayotova. 1997. "Do male reproductive preferences really point to a need to refocus fertility policy?" Population Research and Policy Review 16(5): 447-455.

Eagly, Alice H. and Shelly Chaiken. 1998. "Attitude structure and function," in D. T. Gilbert, S. T. Fiske, and G. Lindzey (eds.), The Handbook of Social Psychology, Volume I. Boston: McGraw-Hill, pp. 269-322.

Easterlin, Richard A. 1975. "An economic framework for fertility analysis," Studies in Family Planning 6(3): 54-63.

El-Zanaty, Fatma, Ann Way, Sunita Kishor, and John Casterline. 1999. Egypt Indepth Study on the Reasons for Nonuse of Family Planning. Cairo: National Population Council.

El-Zeini, Laila O. Forthcoming. "Categorizing the need for family planning: A story of evolution," in S. Szreter, A. Dharmalingam, and H. Sholkamy (eds.), Qualitative Demography: Categories and Contexts in Population Studies. Oxford: Oxford University Press.

Ezeh, Alex Chika. 1993. "The influence of spouses over each other's contraceptive attitudes in Ghana," Studies in Family Planning 24(3): 163-174.

Feyisetan, Bamikale and John B. Casterline. 1999. "Fertility preferences and contraceptive change in developing countries," Policy Research Division Working Paper No. 130. New York: Population Council.

Foreit, Karen and Patricia Mostajo. 1993. "Prevalence is not enough: The importance of appropriateness of contraceptive method in evaluating unmet need for contra- ception," paper presented at the Annual Meeting of the Population Association of America, Cincinnati, April.

Freedman, Ronald, Lolagene C. Coombs, and M. C. Chang. 1972. "Trends in family size 
preferences and practice of family planning: Taiwan, 1965-1970," Studies

in Family Planning 3(12): 281-296.

Freedman, Ronald and Lolagene C. Coombs. 1974. Cross-cultural Comparisons: Data on Two Factors in Fertility Behavior. New York: Population Council.

Garcia-Moreno, Claudia and Ampara Claro. 1994. "Challenges from the women's health movement: Women's rights versus population control," in Gita Sen, Adrienne Germain and Lincoln C. Chen (eds.), Population Policies Reconsidered: Health, Empowerment, and Rights. Cambridge, MA: Harvard University Press, pp. 47-62.

Germain, Adrienne. 1997. "Addressing the demographic imperative through health, empowerment, and rights: ICPD implementation in Bangladesh," Health Transition Review 7(4)[Supp]: 33-36.

Gertler, Paul J. and John W. Molyneaux. 1994. "How economic development and family planning programs combined to reduce Indonesian fertility," Demography 31(1): 33-63.

Hartmann, Betsy. 1987. Reproductive Rights and Wrongs: The Global Politics of Population Control and Contraceptive Choice. New York: Harper and Row.

Hauser, Philip M. 1967. "Family planning and population programs: A book review article," Demography 4(1) 397-414.

1969. "Population: More than family planning," Journal of Medical Education 44 (11, Part 2): 20-29.

Hermalin, Albert I. 1983. "Fertility regulation and its costs: A critical essay," in R. A. Bulatao and R. D. Lee (eds.), The Determinants of Fertility in Developing Coun- tries, Volume 2. New York: Academic Press, pp. 1-53.

Hermalin, Albert I., Ronald Freedman, T. H. Sun, and M. C. Chang. 1979. "Do intentions predict fertility? The experience in Taiwan, 1967-74," Studies in Family Planning 10(3): 75-95.

International Women's Health Coalition (IWHC). 1993. Women's Voices '94-Women's Declaration on Population Policies. New York: IWHC.

Jaccard, James et al. 1990. "Individual differences in attitude-behavior consistency: The prediction of contraceptive behavior," Journal of Applied Social Psychology 20(7): 575-617.

Jain, Anrudh. 1999. "Should eliminating unmet need for contraception continue to be a program priority?" International Family Planning Perspectives 25(Supp): S39-S43.

Jain, Anrudh and Judith Bruce. 1994. "A reproductive health approach to the objectives and assessment of family planning programs," in Gita Sen, Adrienne Germain, and Lincoln C. Chen (eds.), Population Policies Reconsidered: Health, Empow- erment, and Rights. Cambridge, MA: Harvard University Press, pp. 193-209.

Johnson-Acsadi, Gwendolyn and Maurice Szykman. 1984. "Selected characteristics of 'exposed' women who wanted no more children but were not using contraceptives," in J. A. Ross and R. McNamara (eds.), Survey Analysis for the Guidance of Family Planning Programs. Liège: Ordina, pp. 175-217. 
Kelley, Allen C. 1988. "Economic consequences of population change in the Third World," Journal of Economic Literature 27: 1685-1728.

Koenig, Michael A. et al. 1992. "Contraceptive use in Matlab, Bangladesh in 1990: Levels, trends, and explanations," Studies in Family Planning 23(6): 352-364.

Lee, Ronald D. 1990. "Comment: The second tragedy of the commons," Population and Development Review 16 (Supp): 315-322.

Lee, Ronald D., Patrick R. Galloway, and Eugene A. Hammel. 1994. "Fertility decline in Prussia: Estimating influences on supply, demand, and degree of control," Demography 31(2): 347-373.

Lightbourne, R. E. 1985. "Individual preferences and fertility behaviour," in J. Cleland and J. Hobcraft (eds.), Reproductive Change in Developing Countries: Insights from the World Fertility Survey. Oxford: Oxford University Press, pp. 165-198.

Market and Opinion Research International. 1997. Population Issues: Survey Among Key Policy-makers. Report prepared for the Rockefeller Foundation. New York.

Mauldin, W. Parker. 1965. "Fertility studies: Knowledge, attitude, and practice," Studies in Family Planning 1(7): 1-10.

MacKellar, F. Landis. 1997. "Population and fairness," Population and Development Review 23(2): 359-376.

McCauley, P., Brian Robey, Ann Blanc, and J. Geller. 1994. "Opportunities for women through reproductive choices," Population Reports, Series M, no. 12. Baltimore, MD: Johns Hopkins University

McIntosh, C. Alison and Jason L. Finkle. 1995. "The Cairo Conference on Population and Development: A new paradigm?" Population and Development Review 21(2): 223-260.

Mishra, Vinod K., Robert D. Retherford, P. S. Nair, and Griffith Feeney. 1999. "Reasons for discontinuing and not intending to use contraception in India," National Family Health Survey Subject Reports, No. 13. Mumbai, India: International Institute for Population Sciences.

Morris, Leo et al. 1981. "Contraceptive prevalence surveys: A new source of family planning data," Population Reports, Series M, no. 5. Baltimore, MD: Johns Hopkins University.

Mushingeh, Chiponde and Kathleen Kurz. 1998. Unmet Need for Family Planning in Lusaka and Mansa, Zambia. Washington, DC: International Center for Research on Women.

Nag, Moni. 1984. "Some cultural factors affecting costs of fertility regulation," Population Bulletin no. 17. New York: United Nations.

Ngom, Pierre. 1997. "Men's unmet need for family planning: Implications for African fertility transitions," Studies in Family Planning 28(3): 192-202.

Nortman, Dorothy L. 1982. "Measuring the unmet need for contraception to space and limit births," International Family Planning Perspectives 8(4): 125-134.

Nortman, Dorothy L. and Gary L. Lewis. 1984. "A time model to measure contraceptive demand," in J. A. Ross and R. McNamara (eds.), Survey Analysis for the Guidance of Family Planning Programs. Liège: Ordina, pp. 37-73. 
Ochoa, Luis Hernando. 1982. "Prevalence of contraceptive use in Colombia: Determinant factors and policy," in A. I. Hermalin and B. Entwisle (eds.), The Role of Surveys in the Analysis of Family Planning Programs. Liège: Ordina, pp. 85-110.

Omran, A. R., J. Yunes, J. A. Solis, and G. Lopez. 1992. Reproductive Health in the Americas. Washington, DC: Pan American Health Organization.

Palmore, James A. and Mercedes B. Concepción. 1981. "Desired family size and contraceptive use," in World Fertility Survey Conference 1980: Record of Proceedings, Vol. 2. Voorburg, Netherlands: International Statistical Institute, pp. 519-542.

Phai, Nguyen Van, John Knodel, Mai Van Cam, and Hoang Xuyen. 1996. "Fertility and family planning in Vietnam: Evidence from the 1994 Intercensal Demographic Survey," Studies in Family Planning 27(1): 1-17.

Phillips, James F., Cornelius Y. Debpuur, Alex Nazzar, and Elizabeth F. Jackson. 2000.

"The impact of a community health and family planning services experiment on fertility transition in a rural Sahelian population of northern Ghana," paper presented at the Annual Meeting of the Population Association of America, Los Angeles, March.

Phillips, James F. and John A. Ross. 1992. "Family planning programmes and fertility effects: An overview," in J. F. Phillips and J. A. Ross (eds.), Family Planning Programmes and Fertility. Oxford: Clarendon Press, pp. 325-332.

Pittman, Thane S. 1998. "Motivation," in D. T. Gilbert, S. T. Fiske, and G. Lindzey (eds.), The Handbook of Social Psychology, Volume I. Boston: McGraw-Hill, pp. 549-590.

Population Council/Islamabad. 1997. The Gap Between Reproductive Intentions and Behaviour: A Study of Pakistani Men and Women. Islamabad: Population Council.

Pritchett, Lant H. 1994. "Desired fertility and the impact of population policies," Population and Development Review 20(1): 1-55.

Pullum, Thomas W., George E. Immerwahr, and Josefina V. Cabigon. 1984. "Fertility preferences and contraceptive use in the Philippines," in J. A. Ross and R. McNamara (eds.), Survey Analysis for the Guidance of Family Planning Programs. Liège: Ordina, pp. 147174.

Raftery, Adrian E., Steven M. Lewis, and Akbar Aghajanian. 1995. "Demand or ideation? Evidence from the Iranian marital fertility decline," Demography 32(2): 159-182.

Ravenholt, R. T. and John Chao. 1974. "Availability of family planning services: The key to rapid fertility reduction," Family Planning Perspectives 6(4): 217-223.

Robey, Bryant, John Ross, and Indu Bhushan. 1996. "Meeting unmet need: New strategies," Population Reports, Series J, no. 43. Baltimore, MD: Johns Hopkins University.

Ross, John. 1994. "Program implementation of the unmet need approach." paper pre- sented at the Annual Meeting of the Population Association of America, Miami, May.

—. 1995. "The question of access," Studies in Family Planning 26(4): 241-242.

Ross, John and Laura Heaton. 1997. "Intended contraceptive use among women without an unmet 
need," International Family Planning Perspectives 23(4): 148-154.

Ross, John, John Stover, and Amy Willard. 1999. Profiles for Family Planning and Reproductive Health Programs. Glastonbury, CT: The Futures Group.

Sai, Fred T. 1997. "The ICPD Programme of Action: Pious hope or workable guide?" Health Transition Review 7(4) [Supp]: 1-5.

Sen, Gita, Adrienne Germain, and Lincoln C. Chen (eds.). 1994. Population Policies Reconsidered: Health, Empowerment, and Rights. Cambridge, MA: Harvard University Press.

Shah, Iqbal H. and John G. Cleland. 1993. "High fertility in Bangladesh, Nepal, and Pakistan: Motives vs. means," in Richard Leete and Iqbal Alam (eds.), The Revolution in Asian Fertility: Dimensions, Causes, and Implications: Oxford: Clarendon Press, pp. 175-207.

Shelton, James D. et al. 1999. "Putting unmet need to the test: Community-based distribution of family planning in Pakistan," International Family Planning Perspec- tives 25(4): 191-195.

Short, R. V. 1994. "Contraceptive strategies for the future," in F. Graham-Smith (ed.), Population: The Complex Reality. Golden, CO: North American Press, pp. 323339.

Sinding, Steven W., John A. Ross, and Allan G. Rosenfield. 1994. "Seeking common ground: Unmet need and demographic goals," International Family Planning Perspectives 20(1): 23-27, 32.

Stash, Sharon. 1999. "Explanations of unmet need for contraception in Chitwan, Ne- pal," Studies in Family Planning 30(4): 267-287.

Stokes, B. 1977. "Filling the family planning gap," World Watch Paper 12. Washington, DC: World Watch Institute.

Tan, Poo Chang and Nai Peng Tey. 1994. "Do fertility intentions predict subsequent behavior? Evidence from Peninsular Malaysia," Studies in Family Planning 25(4): 222-231.

United Nations. 1974. World Population Plan of Action. New York.

- 1984. Report of the International Conference on Population, 1984. New York. 1994. Programme of Action of the International Conference on Population and Development. New York. 1999. Levels and Trends of Contraceptive Use as Assessed in 1998. New York. US

Commission on Population Growth and the American Future. 1972. Population and the American Future. Washington, DC: US Government Printing Office.

Viswanathan, Hema, Sona Godfrey, and Nancy Yinger. 1998. Reaching Women: A Study of Unmet Need in Uttar Pradesh, India. Washington, DC: International Center for Research on Women.

Westoff, Charles F. 1978. "The unmet need for birth control in five Asian countries," Family Planning Perspectives 10(3): 173-181. 
1988. "The potential demand for family planning: A new measure of unmet need and estimates for five Latin American countries," International Family Planning Perspectives 14(2): 45-53.

1990. "Reproductive intentions and fertility rates," International Family Planning Perspectives 16(3): 84-89, 96.

Forthcoming. "Unmet need revisited," in A. Tsui, J. Phillips, and L. Rosero- Bixby (eds.), Methods for Evaluating Family Planning Programs. Oxford: Ox- ford University Press.

Westoff, Charles F. and Akinrinola Bankole. 1995. Unmet Need: 1990-1994. Demographic and Health Surveys Comparative Studies No. 16. Calverton, MD: Macro International Inc.

_. 1996. "The potential demographic significance of unmet need," International Family Planning Perspectives 22(1): 16-20.

. 1998. "The time dynamics of unmet need: An example from Morocco," International Family Planning Perspectives 24(1): 12-14.

Westoff, Charles F. and Luis H. Ochoa. 1991. Unmet Need and the Demand for Family Planning. Demographic and Health Surveys Comparative Studies No. 5. Calverton, MD: Macro International Inc.

Westoff, Charles F. and Anne R. Pebley. 1981. "Alternative measures of unmet need for family planning in developing countries," International Family Planning Perspectives 7(4): 126136.

Westoff, Charles F. and Norman B. Ryder. 1977. "The predictive validity of reproductive intentions," Demography 14(4): 431-453.

Yinger, Nancy V. 1998. Unmet Need for Family Planning: Reflecting Women's Perceptions. Washington, DC: International Center for Research on Women. 


\title{
POLICY RESEARCH DIVISION WORKING PAPERS
}

\author{
Recent Back Issues
}

1998

*106 Sajeda Amin and Gilda Sedgh, "In- centive schemes for school atten- dance in rural Bangladesh."

107 Martin Brockerhoff and Paul Hewett, "Ethnicity and child mortal- ity in sub-Saharan Africa."

108 Ann E. Biddlecom and Bolaji M. Fapohunda, "Covert contraceptive use: Prevalence, motivations, and consequences."

109 John Bongaarts and Griffith Feeney,

"On the quantum and tempo of fer- tility."

110 Barbara S. Mensch, Daniel Bagah, Wesley H. Clark, and Fred Binka,

"The changing social environment for

adolescents in the Kassena-Nankana District of northern Ghana: Implica- tions for reproductive behavior."

111 Martin Brockerhoff and Ann Biddle- com, "Migration, sexual behavior, and HIV diffusion in Kenya."

112 Zeba A. Sathar and John B. Caster- line, "The onset of fertility transition in Pakistan."

113 Geoffrey McNicoll, "Government and fertility in transitional and post- transitional societies."

114 John Bongaarts, "Fertility and repro- ductive preferences in post-transi- tional societies."

115 Fiona Steele, Sajeda Amin, and Ruchira T. Naved, "The impact of an integrated micro-credit program on women's empowerment and fertility behavior in rural Bangladesh."

*116 Cynthia B. Lloyd, Barbara S. Mensch, and Wesley H. Clark, "The effects of primary-school quality on the educational participation and at- tainment of Kenyan girls and boys."

117 Sajeda Amin and Cynthia B. Lloyd,

"Women's lives and rapid fertility de- cline: Some lessons from Bangladesh and Egypt."

118 James F. Phillips and Mian Bazle Hossain, "The impact of family planning household service delivery on women's status in Bangladesh."

119 Mark R. Montgomery and John B. Casterline, "Social networks and the diffusion of fertility control."

* No longer available 
*120 John Bongaarts, "The fertility impact of changes in the timing of childbear- ing in the developing world."

*121 James F. Phillips, Wendy L. Greene, and Elizabeth F. Jackson, "Lessons from community-based distribution of family planning in Africa."

122 Mark R. Montgomery, "Mortality de- cline and the demographic response: Toward a new agenda."

123 Mark R. Montgomery, Mary Arends- Kuenning, and Cem Mete, "The quantity-quality transition in Asia."

124 Barbara S. Mensch, Wesley H. Clark, Cynthia B. Lloyd, and Annabel S. Erulkar, "Premarital sex and school dropout in Kenya: Can schools make a difference?"

125 John Bongaarts and Rodolfo A. Bulatao, "Completing the demo- graphic transition."

126 Geoffrey McNicoll, "Population weights in the international order."

127 Cynthia B. Lloyd, Carol E. Kaufman, and Paul Hewett, "The spread of primary schooling in subSaharan Africa: Implications for fertility change."

128 John B. Casterline, "The onset and pace of fertility transition: National patterns in the second half of the twentieth century."

129 Mark R. Montgomery, Michele Gragnolati, Kathleen Burke, and Ed- mundo Paredes, "Measuring living standards with proxy variables."

130 Bamikale Feyisetan and John B. Casterline, "Fertility preferences and contraceptive change in devel- oping countries."

131 Martin Brockerhoff, "Urban growth in developing countries: A review of projections and predictions."

132 Omaima El-Gibaly, Barbara Ibrahim, Barbara S. Mensch, and Wesley H. Clark, "The decline of female circumcision in Egypt: Evidence and inter- pretation."

* No longer available 
2000

133 Mary Arends-Kuenning and Sajeda Amin, "The effects of schooling in- centive programs on household re- source allocation in Bangladesh."

134 John Bongaarts and Charles F. Westoff, "The potential role of con- traception in reducing abortion."

135 John B. Casterline and Steven W.Sinding, "Unmet need for family planning in developing countries and implications for population policy." 